\title{
Thiol modified mycolic acids
}

Mohammed O. Balogun, ${ }^{1}$ Enlli Huws, ${ }^{2}$ Muthana M. Sirhan, ${ }^{2}$ Ahmed D. Saleh, ${ }^{2}$ Juma'a R. Al Dulayymi, ${ }^{2}$ Lynne Pilcher, ${ }^{1}$ Jan A.Verschoor, ${ }^{3}$ Mark S. Baird ${ }^{2 *}$

${ }^{a}$ Department of Chemistry, University of Pretoria, South Africa

${ }^{\mathrm{b}}$ School of Chemistry, Bangor University, Bangor, Wales, LL57 2UW, United Kingdom

${ }^{\mathrm{c}}$ Department of Biochemistry, University of Pretoria, South Africa

* Corresponding author. Tel.: +44 1248 382374; fax: +44 01248370528.

E-mail address: chs028@bangor.ac.uk (M.S. Baird).

\section{Highlights}

The synthesis of thiol modified mycobacterial mycolic acids is reported.

Cyclic voltammetry shows these will bind to a gold surface.T

This offers the potential for improved diagnosis of tuberculosis in antibody-antigen assays.

\begin{abstract}
Patient serum antibodies to mycolic acids have the potential to be surrogate markers of active tuberculosis (TB) when they can be distinguished from the ubiquitously present cross-reactive antibodies to cholesterol. Mycolic acids are known to interact more strongly with antibodies present in the serum of patients with active TB than in patients with latent TB or no TB. Examples of single stereoisomers of mycolic acids with chain lengths corresponding to major homologues of those present in Mycobacterium tuberculosis have now been synthesised with a sulfur substituent on the terminal position of the $\alpha$-chain; initial studies have established that one of these binds to a gold electrode surface, offering the potential to develop second generation sensors for diagnostic patient antibody detection.
\end{abstract}




\section{Graphical abstract:}

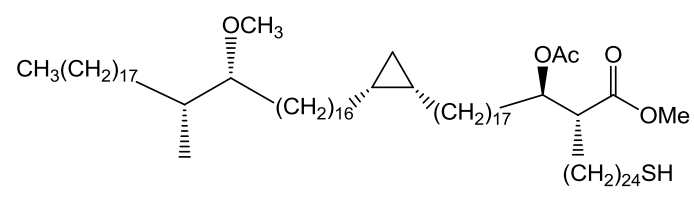

Keywords: Mycolic acids; Thiolated; Gold surfaces; Cyclic voltammetry

Mycolic acids (1) such as (1)-(4) (Scheme 1) are characteristic components of the cell walls of mycobacteria and a number of other species such as Norcardia and Rhodococcus [Minnikin, 1982; Barry et al, 1998; Verschoor et al, 2012]. These long chain $\beta$-hydroxy acids have a simple hydrocarbon chain in the $\alpha$ position. The parent chain often contains two functional groups. Thus the mycolic acids of Mycobacterium tuberculosis are classified into three major types based on these functional groups, $\alpha$ MA (1), methoxy-MA (2) and keto-MA (3). In general, the cyclopropanes have a cisstereo-chemistry; when a trans-cyclopropane is present it has a methyl substituent on the adjacent carbon distal from the hydroxy acid. Mycolic acids are present in cells as complex mixtures of different homologues. They are present either as free mycolic acids, or as sugar esters such as trehalose esters, but are mainly bound to the bacterial cell wall by esterification to arabinogalactan. They are isolated from cells as complex mixtures of different classes and different chain lengths [Watanabe et al, 2001; 2002]. The make-up of these mixtures is characteristic of the particular mycobacterium and detailed finger-printing of the mass spectra of such mixtures can be used directly to identify the mycobacterium present in a sample and hence the presence of a disease such as tuberculosis [Uenishi et al, 2008; Salvado-Viader et al, 2007; Yassin, 2011; Shui et al, 2012; Kowalski et al, 2012; Song et al, 2009; Lval et al, 2001]. The presence of particular patterns of mycolic acid have also been used to characterise a new genus of bacteria [Hong et al, 2012]. 

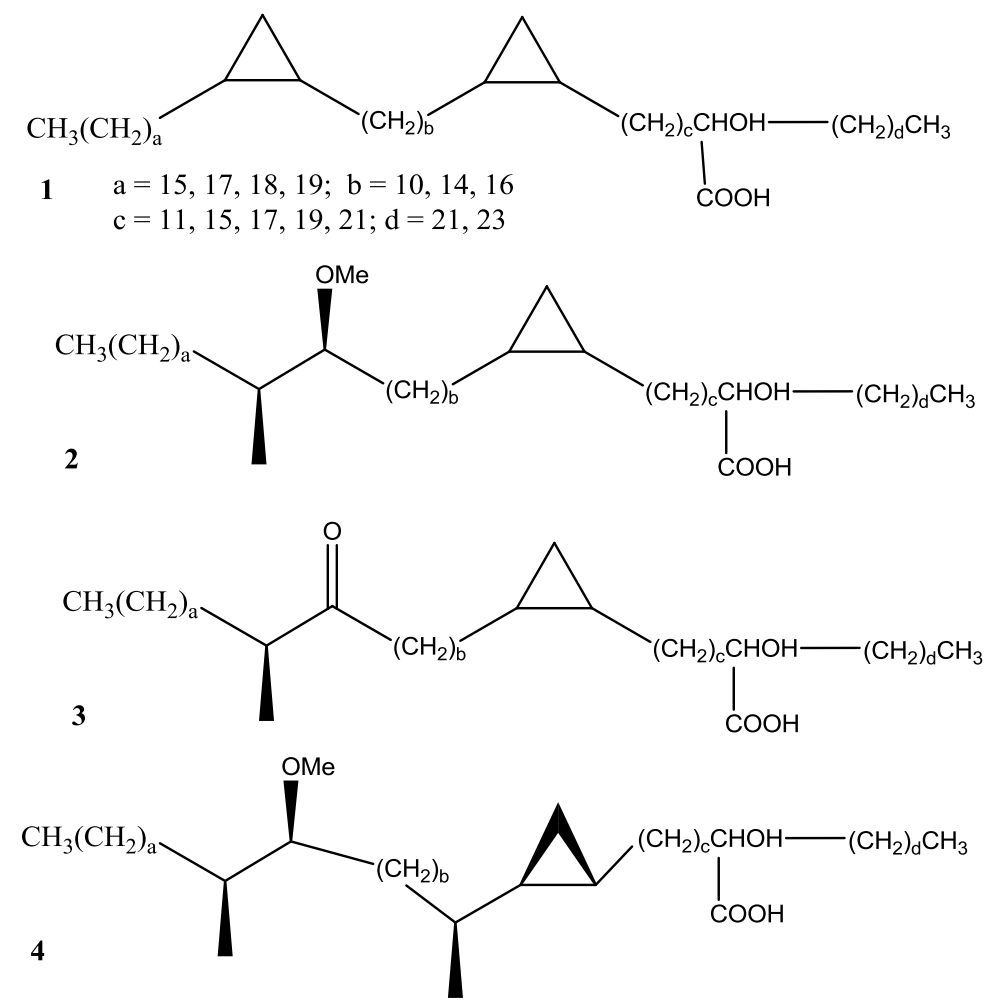

Scheme 1: Common classes of mycolic acids in mycobacteria

Mixtures of trehalose esters of mycolic acids have been used in ELISA assays to detect the presence of antibodies in the serum of infected patients and to distinguish this from infection by Mycobacterium avium [Maekura et al, 1993; Sakai et al, 2001; Fujiwara et al, 1999; Pan et al, 1999]. Despite very positive results, this assay and a large number of related serodiagnostic assays have been found not to reach the required levels of specificity and sensitivity [Steingart et al, 2007; 2009; Morris, 2011; Ireton, 2010 ].

Mixtures of free mycolic acids isolated from the cells of $M$. tuberculosis have also been used in serodiagnostic assays for the disease. In the simplest form they are used to detect interactions with antibodies present in the serum of infected patients using an ELISA assay. Although this is a very rapid approach to the diagnosis of $\mathrm{TB}$, the sensitivity and specificity are again not high enough - i.e., there are too many false negatives and too many false positives [Beukes et al, 2011; Verschoor, 2010]. Part of the problem in this case may be that some of the antibodies present are cross-reactive to mycolic acids and cholesterol [Benadie, 2008]. In order to seek to improve sensitivity and specificity, Verschoor et al have developed a method based on the use 
of mycolic acid containing liposomes in an inhibition assay using surface Plasmon resonance [Verschoor, 2010; Thanyani et al, 2008; Lemmer et al, 2009]. One singular advantage of this method is that the signals are retained in HIV patients [Schleicher et al, 2002].

In order to explore electrochemical methods for immunosensing, Ozoemena et. al. modified a gold surface with a thiolated fatty acid and integrated mycolic acids into the hydrophobic layer. Using electrochemical impedence spectroscopy (EIS), they showed that it is possible to distinguish TB+ from TB- sera [Ozoemena et al, 2010; Mathebula et al, 2009]. In order to optimise the use of myolic acids in such diagnostic devices for TB, we wish to bind them to gold by modifying them to contain a thiol group. Since it is only possible to modify the natural isolated mixture of mycolic acids by reaction at functional groups and in so doing interfere with antibody binding, suitably thiolated mycolic acids are only accessibly via total synthesis. We propose that the terminus of the invariant alpha-alkyl chain is the best place to introduce the thiol moiety without disrupting biological activity.

We have already reported the synthesis of mycolic acids with the same structures and chain lengths as the major homologues present in natural mixtures; in each case the molecule has been prepared as a single enantiomer [Al Dulayymi et al, 2003, 2005, 2006a, 2007]. We now report the preparation of four such molecules, in each case modified by the introduction of a thiol substituent at the end of the $\alpha$-carbon chain of the meromycolate fragment. This position for thiolation was chosen in order to minimise conformational changes around the $\beta$-hydroxy acid and its interaction with the long functionalised chain.

In the first series of experiments, a model thiolated $\beta$-hydroxy acid (13) was prepared: A modified Julia-Kocienski coupling of the aldehyde (5) with sulfone (6), followed by hydrogenation of the derived E/Z-mixture of alkenes led to the protected $\beta$ hydroxy acid fragment (7) with a fourteen carbon $\alpha$-chain. This could be converted into the thioacetate (9) via the alcohol (8) using a two step procedure of tosylation followed by thioacetate substitution. 


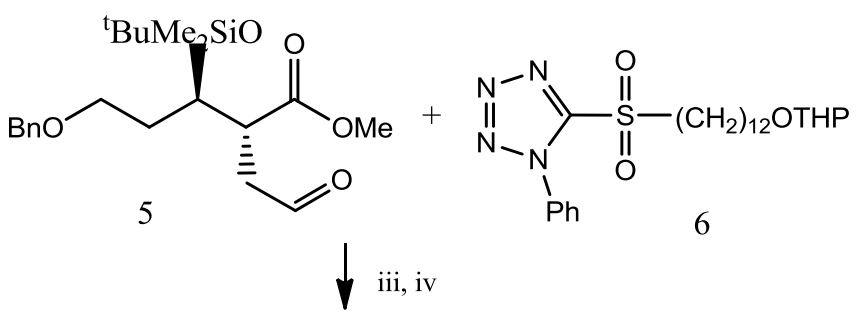<smiles>COC(=O)[C@H](C)[C@@H](CCO)O[Mg]</smiles>

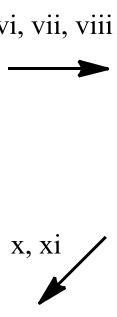<smiles>CCO[C@@H](CC)[C@@H](C)C(=O)OC</smiles><smiles>CC[C@@H](OC)[C@@H](C)C(=O)OC</smiles>

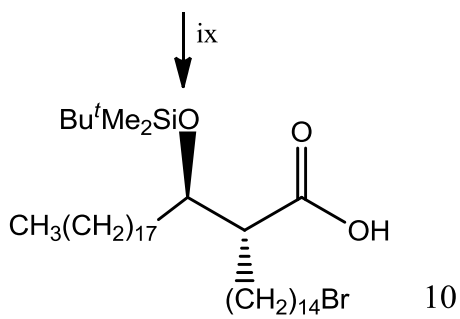<smiles>C[14CH3]</smiles><smiles>CC[C@H](O)[C@@H](C)C(=O)OC(C)=O</smiles>
11

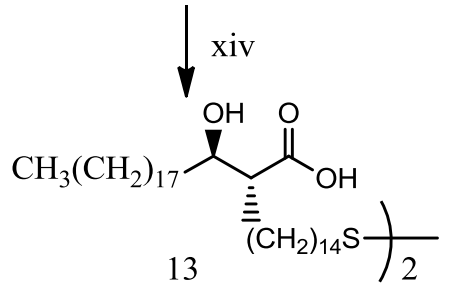

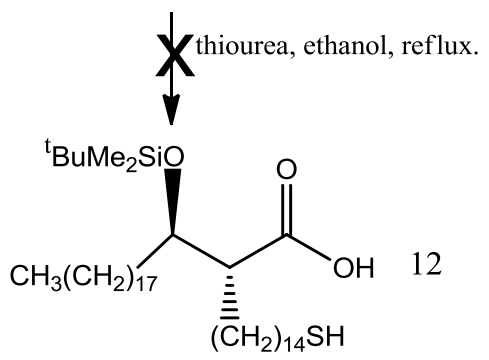<smiles>CCC(C)(C)C</smiles>

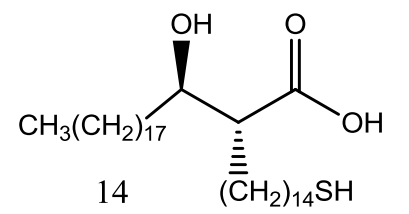

Scheme 2: (iii) LiHMDS, THF, $75 \%$; (iv) $\mathrm{H}_{2}, \mathrm{Pd} / \mathrm{C}$, 79\%; (v) PCC, $82 \%$; (vi) LiHMDS, 5(hexadecone-1-sulfonyl)-1-phenyl-14-tetrazole, THF, $75 \%$; (vii) $\mathrm{H}_{2}$, Pd/C $92 \%$; (viii) pyridinium-ptoluene sulphonate, MeOH/THF, 82\%; (ix) N-bromosuccinimide, $\mathrm{PPh}_{3}, \mathrm{CH}_{2} \mathrm{Cl}_{2}, 72 \%$; (x) TsCl, $\mathrm{Et}_{3} \mathrm{~N}$, $74 \%$; (xi) potassium thioacetate, acetone, $75 \%$; (xii) pyridine, HF-pyridine, $84 \%$; (xiii) LiOH. $\mathrm{H}_{2} \mathrm{O}$ (15.eq), THF/MeOH/ $\mathrm{H}_{2} \mathrm{O}$; (xiv) $\mathrm{LiOH} . \mathrm{H}_{2} \mathrm{O}$ (4.eq), THF/MeOH/ $\mathrm{H}_{2} \mathrm{O}, 26 \%$.

Deprotection of (9) was achieved in two steps. The final deprotection gave the disulphide (13) rather than the free thiol (14), as indicated by the mass ion in the MALDI MS and by a triplet for the methylene group adjacent to sulphur at $\delta 2.70$ in the proton NMR spectrum and a signal for the same methylene carbon at $\delta 40$ in the carbon NMR spectrum. Although this was prepared simply as a model, it does represent a thiol substituted form of one of the minor mycolic acid components of Rhodococcus equi [Hsu, 2011] 
In order to prepare a complete thiolated mycolic acid, the intermediate (19) was first prepared. Coupling of the aldehyde (5) [Koza, 2009] with the sulfone (15) in the presence of base in a modified Julia-Kocienski reaction, followed by hydrogenation of the E/Z-mixture of alkenes obtained, then debenzylation, led to alcohol (16). This was chain extended using a similar procedure to give the aldehyde (19) (Scheme 2):

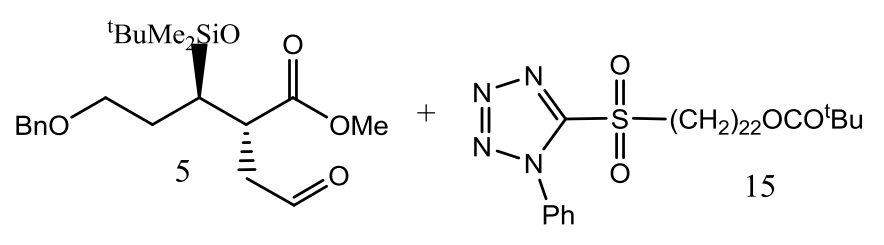<smiles>COC(=O)[C@H](C)[C@@H](CCO[13CH3])O[13CH3]</smiles>

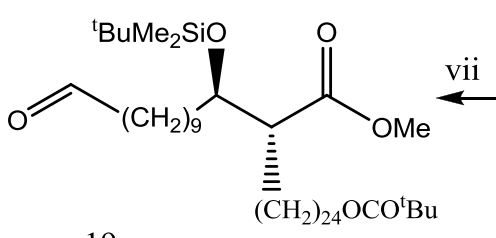

19<smiles>COC(=O)[C@H](C)[C@@H](CO)OCCO</smiles>

Scheme 2: (i) LiHMDS, THF, $67 \%$; (ii) $\mathrm{H}_{2}$, Pd/C $68 \%$; (iii) PCC, $82 \%$; (iv) LiHMDS, 1-phenyl-5((8-((tetrahydro-2H-pyran-2-yl)oxy)octyl)sulfonyl)-1H-tetrazole, $85 \%$; (v) pyridinium p-toluene sulphonate, $\mathrm{MeOH} / \mathrm{THF}, 88 \%$; (vi) $\mathrm{H}_{2}$, Pd/C $88 \%$; (vii) PCC, $84 \%$.

Coupling of this aldehyde (19) to the known sulfone (20) [Al Dulayymi, 2007] in the presence of base followed by hydrogenation led to alcohol (21) (Scheme 3). This was converted into the corresponding thioacetate (22); the transformation of alcohol to thioacetate was confirmed by the replacement of a triplet the methylene group adjacent to oxygen at $\delta_{\mathrm{H}} 3.7$ with that adjacent to the thioacetate at $\delta 2.86$, and the loss of the corresponding carbon signal at $\delta_{\mathrm{C}} 63.1$. This ${ }^{13} \mathrm{C}$ methyl signal in the thioacetate appears in the region 28-34, together with other signals of the MA, but the formation of the thioacetate is confirmed by an additional carbonyl carbon at $\delta_{C} 196.1$. 


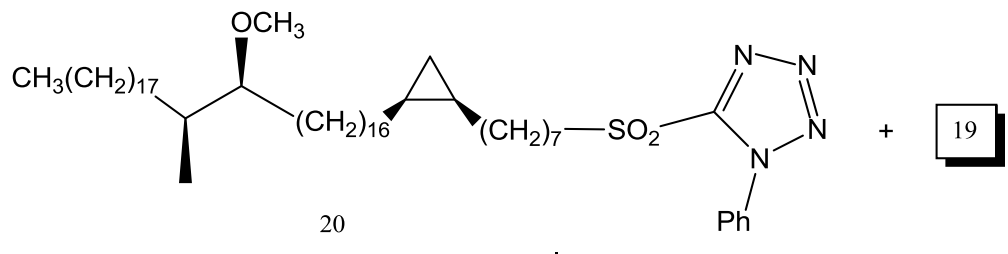

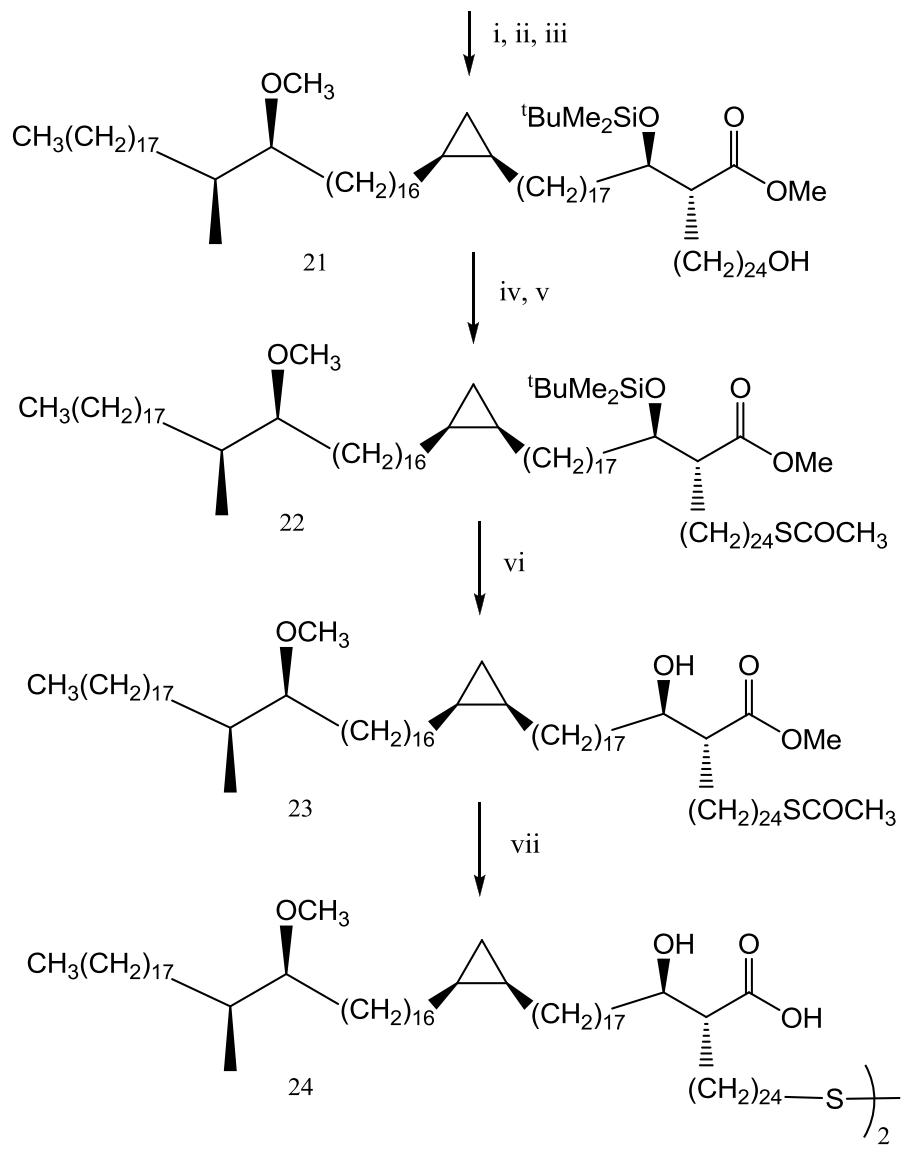

Scheme 3: (i) LiHMDS, THF, $71 \%$; (ii) $\mathrm{KOOCN}=\mathrm{NCOOK}, \mathrm{AcOH} / \mathrm{MeOH} / \mathrm{THF}, 78 \%$; (iii) $\mathrm{KOH}$, THF, $\mathrm{MeOH}, \mathrm{H}_{2} \mathrm{O}, 70 \%$; (iv) TsCl, $\mathrm{Et}_{3} \mathrm{~N}, \mathrm{CH}_{2} \mathrm{Cl}_{2}, 75 \%$; (v) potassium thioacetate, THF, acetone, 76 $\%$; (vi) HF, pyridine, THF, $63 \%$; (vii) tetrabutylammonium hydroxide $(5 \%), 100{ }^{\circ} \mathrm{C}, 18 \mathrm{~h}, 26 \%$.

The final deprotection of (22) to give the free thio-substituted mycolic acid proved to be very sensitive to reaction conditions. The free thiol was not isolated and the disulfide (24) was isolated in only $26 \%$ yield. The ${ }^{1} \mathrm{H}$ chemical shift of the methylene group adjacent to sulfur was $\delta 2.76$ for $(\mathbf{2 4})$ and the corresponding ${ }^{13} \mathrm{C}$ resonated at $\delta$ $39.3 \mathrm{ppm}$. Unfortunately the disulfide did not give the expected molecular ion in MALDI MS. The resolution of this latter problem is described later.

In order to study the effect of the absolute stereochemistry of the mycolic acid on its use in diagnostics, a second diastereoisomer was prepared using the same procedure (Scheme 4): 


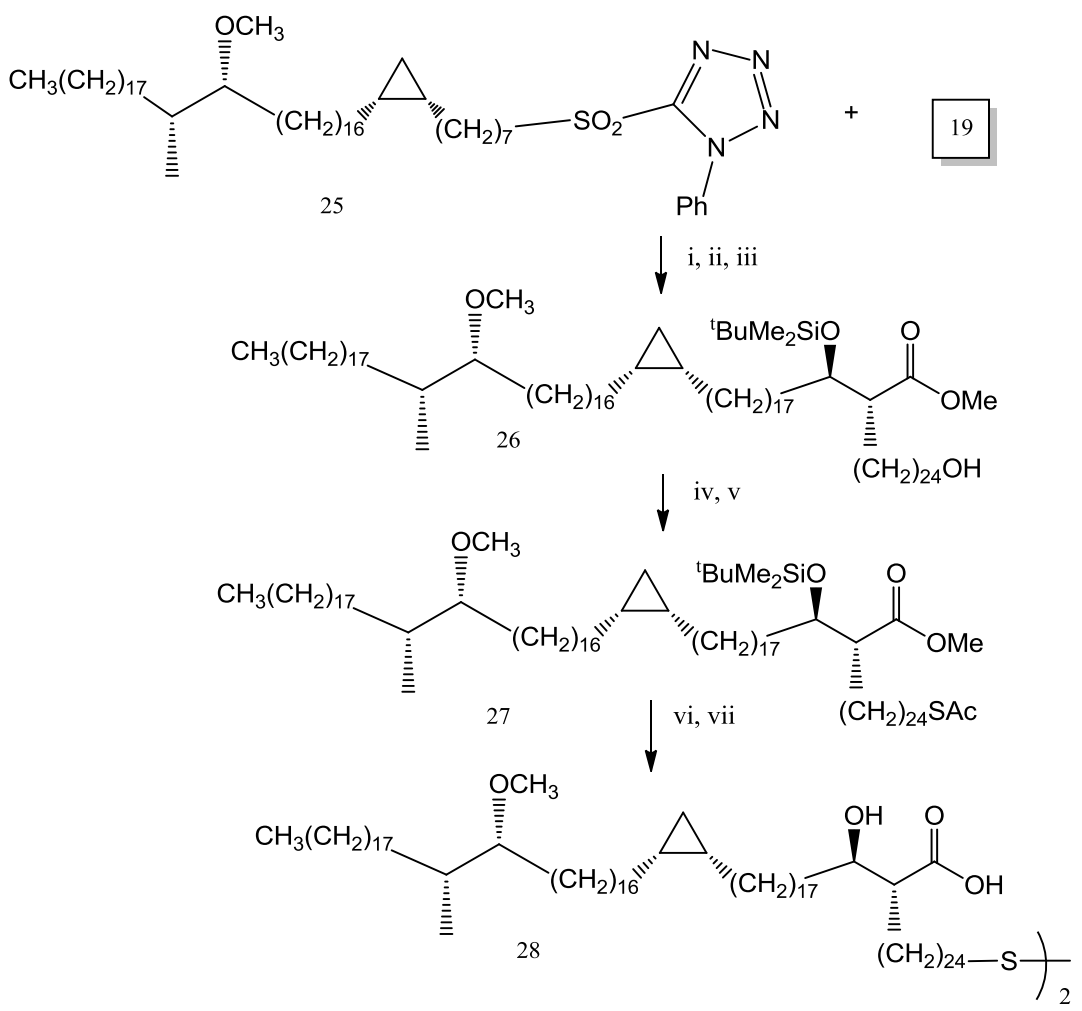

Scheme 4: (i) LiHMDS, THF, 89 \%; (ii) $\mathrm{KOOCN}=\mathrm{NCOOK}$, $\mathrm{AcOH} / \mathrm{MeOH} / \mathrm{THF}, 93 \%$; (iii) $\mathrm{KOH}$, THF, $\mathrm{MeOH}, \mathrm{H}_{2} \mathrm{O}, 60 \%$; (iv) TsCl, $\mathrm{Et}_{3} \mathrm{~N}, \mathrm{CH}_{2} \mathrm{Cl}_{2}, 74 \%$; (v) potassium thioacetate, THF, acetone, 82 $\%$; (vi) HF, pyridine, THF, $97 \%$; (vii) tetrabutylammonium hydroxide $(5 \%), 100{ }^{\circ} \mathrm{C}, 18 \mathrm{~h}, 54 \%$.

In this case, the final deprotection gave the disulfide (28), which again did not provide a molecular ion. As in the case of its diastereomer (24), the methylene group adjacent to sulfur appeared at $\delta$ 2.7. The carbons adjacent to sulfur in (24) and (28) appeared at 39.3 and 39.1 respectively. Compound (28) also showed four signals in the carbon spectrum at $\delta 85.6,72.1,57.7$ and 51 characteristic of the $\mathrm{CH}(\mathrm{OMe}), \mathrm{CH}(\mathrm{OH}), \mathrm{OCH}_{3}$ and $\mathrm{CH}(\mathrm{COOH})$ carbons as seen in the parent methoxymycolic acid with no sulfur substituent on the $\alpha$-chain [Al Dulayymi, 2007]. There were also signals at $\delta_{\mathrm{C}} 10.9$ $\left(\mathrm{CH}_{2}\right)$ and $15.8(2 \mathrm{x} \mathrm{CH})$ for the cyclopropane carbons as well as a characteristic signal at $\delta_{\mathrm{C}} 22.5$ present in all mycolic acids, but no signal at $\delta_{\mathrm{C}} 24-26$.

In order to fully characterize the disulfides, the acid (28) was esterified with diazomethane and the alcohol was reprotected as an acetate. The disulfide was then reductively cleaved to give the thiol (29) using DL-dithiothreitol. This gave the expected molecular ion in MALDI MS and the methylene group adjacent to sulfur appeared as a quartet at $2.53(J 7.52 \mathrm{~Hz})$. In addition the carbon signal for the 
methylene group adjacent to sulphur had shifted from around $\delta_{\mathrm{C}} 39.5$ in the disulphide to 24.7 in the thiol. This is typical for the carbon adjacent to sulfur in sulfides.[Anklam, 1990; Thuo, 2011; Angelova, 2005]

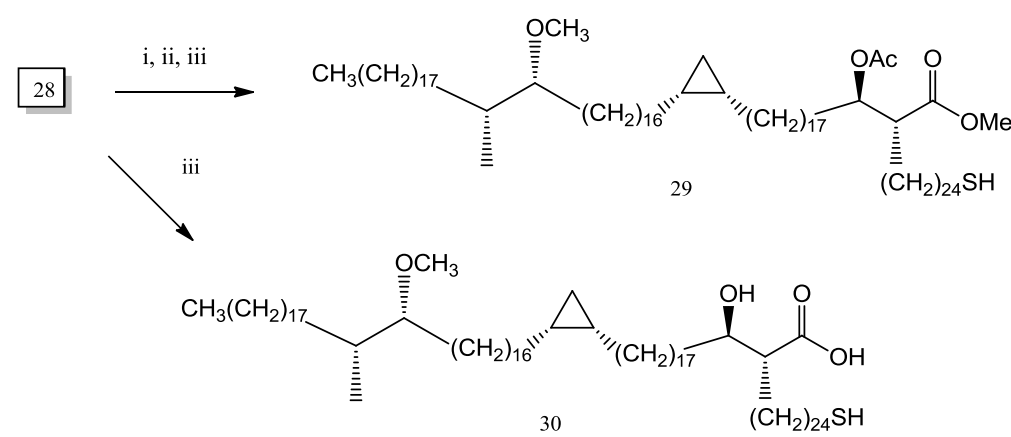

Scheme 5: (i) excess diazomethane; (ii) acetic anhydride, pyridine, toluene; (iii) DL-dithiothreitol

In the same way, the disulfide could be split to free thiol (30) without protection of the alcohol and acid groups. The ${ }^{1} \mathrm{H}$ NMR spectrum of this again showed a quartet at $\delta 2.52$ for the methylene group adjacent to sulphur. When the sample was shaken with $\mathrm{D}_{2} \mathrm{O}$, this became a triplet with $\mathrm{J} 7.2 \mathrm{~Hz}$. Once again, the methylene carbon adjacent to sulfur had shifted from $\delta_{\mathrm{C}} 39.5$ to 24.6 . This was confirmed by a proton carbon correlation.

The formation of the diastereoisomer (24) could also be confirmed by formation of the thiol (31).

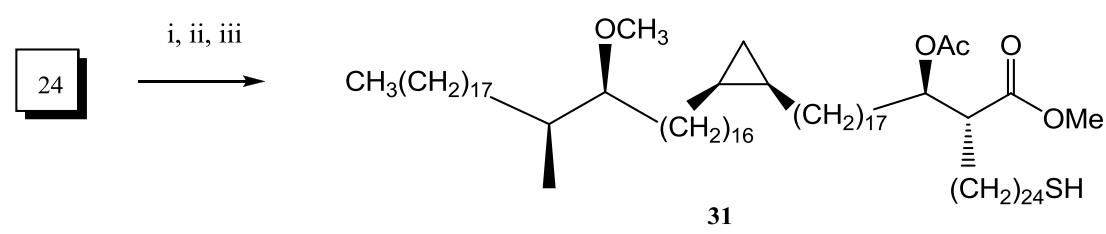

Scheme 6: (i) excess diazomethane; (ii) acetic anhydride, pyridine, toluene; (iii) DL-dithiothreitol

In the second part of this work, the same methods were applied to the synthesis of a thiol-substituted methoxymycolic acid containing a trans- $\alpha$-methylcyclopropane. 

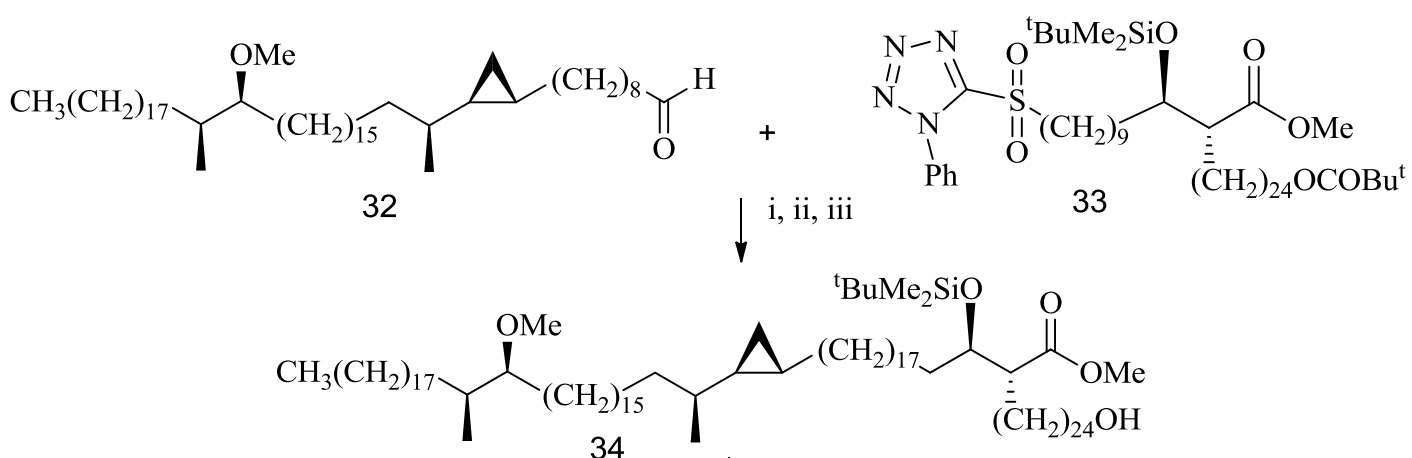

34

iv, $\mathrm{v}$
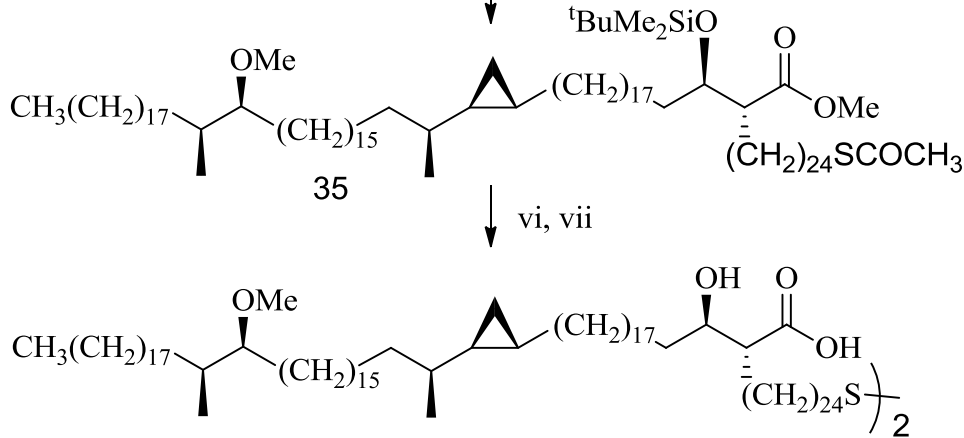

36

Scheme 7: (i) LiHMDS, THF, $86 \%$; (ii) $\mathrm{KOOCN}=\mathrm{NCOOK}$, $\mathrm{AcOH} / \mathrm{MeOH} / \mathrm{THF}, 86 \%$; (iii) $\mathrm{KOH}$, THF, MeOH, $\mathrm{H}_{2} \mathrm{O}, 86 \%$; (iv) $\mathrm{TsCl}, \mathrm{Et}_{3} \mathrm{~N}, \mathrm{CH}_{2} \mathrm{Cl}_{2}, 65 \%$; (v) potassium thioacetate, THF, acetone, 61 $\%$; (vi) HF, pyridine, Pyridine, THF, $90 \%$; (vii) tetrabutylammonium hydroxide $(5 \%), 100{ }^{0} \mathrm{C}, 18 \mathrm{~h}$, $58 \%$.

In this case, the aldehyde (32), containing a stereodefined $\alpha$-methyl-trans-cyclopropane fragment [Al Dulayymi et al, 2103] was coupled to sulfone (33), prepared by a standard route. Saturation of the derived mixture of alkene stereoisomers gave the alcohol (34) which was converted into the thioacetate (35) using the same method as above. Once again, removal of the protecting groups led to the formation of the disulfide (36) rather than the free thiol; seen by the presence of signals at $\delta_{\mathrm{H}} 2.69(J$ $7.4)$ and $\delta_{\mathrm{C}} 39.5$ for the methylene group adjacent to the sulfur.

Finally a thiolated $\alpha$-mycolic acid was prepared. The sulfone (37) was prepared by the same route as that reported for its enantiomer [Al Dulayymi, 2005]. Coupling to the aldehyde (19) in a modified Julia Kocienski reaction, followed by saturation of the derived alkenes gave (38). Removal of the pivaloate protecting group led to (39), which was transformed as before into the acetate (40). Once again, deprotection led to the corresponding disulfide (41). 


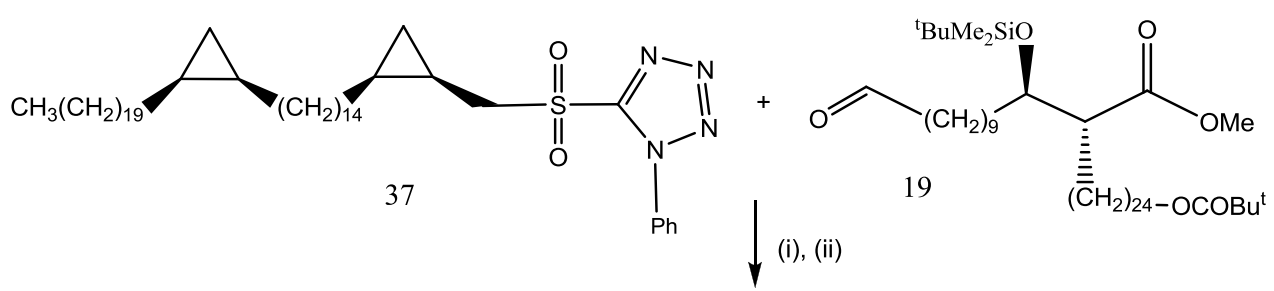

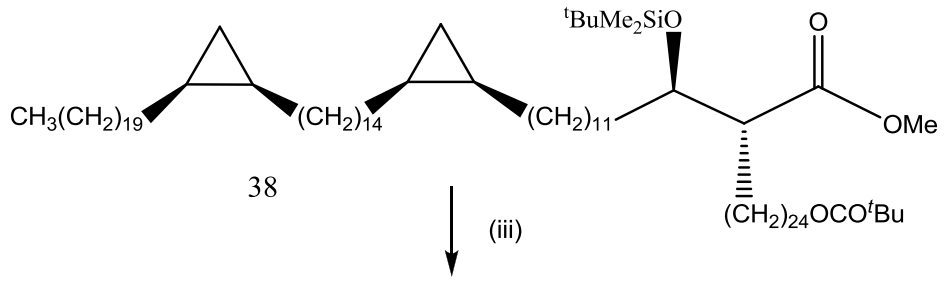

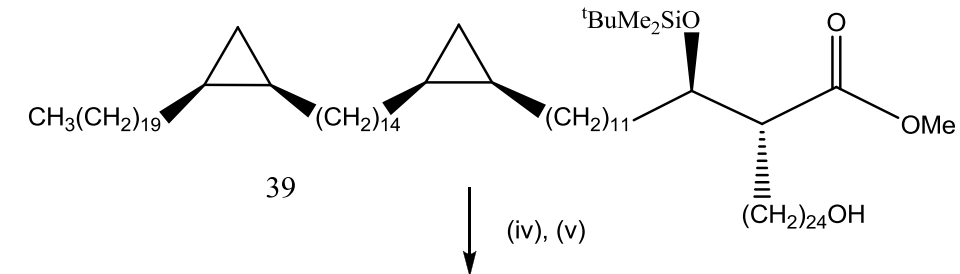

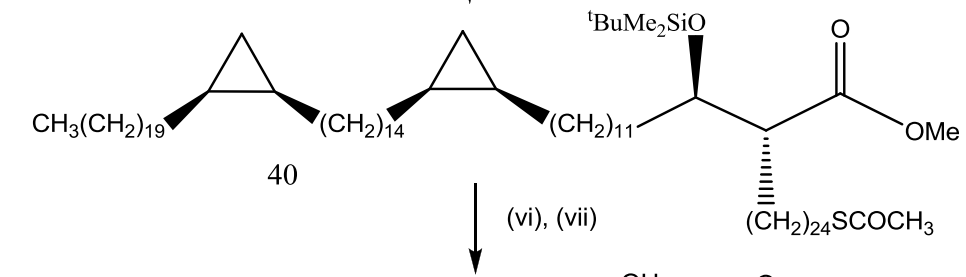

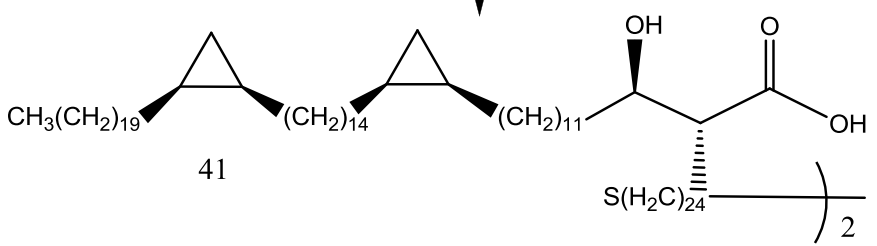

Scheme 11: (i) LiHMDS, THF, $60 \%$; (ii) $\mathrm{KOOCN}=\mathrm{NCOOK}, \mathrm{AcOH} / \mathrm{MeOH} / \mathrm{THF}, 91 \%$; (iii) $\mathrm{KOH}$, THF, $\mathrm{MeOH}, \mathrm{H}_{2} \mathrm{O}, 67 \%$; (iv) $\mathrm{TsCl}, \mathrm{Et}_{3} \mathrm{~N}, \mathrm{CH}_{2} \mathrm{Cl}_{2}, 71 \%$; (v) potassium thioacetate, THF, acetone, 94 $\%$; (vi) HF, pyridine, THF, $55 \%$; (vii) tetrabutylammonium hydroxide $(5 \%), 100{ }^{0} \mathrm{C}, 18 \mathrm{~h}, 33 \%$.

The formation of the disulfide was seen by the presence of a characteristic carbon signal at $\delta_{\mathrm{C}} 39.2$ for the methylene group adjacent to sulfur, and the corresponding proton signal at $\delta_{\mathrm{H}} 2.67$. This was again confirmed by protection of the alcohol and acid groups followed by the reduction of the disulfide using DL-dithiothreitol to give thiol (42):

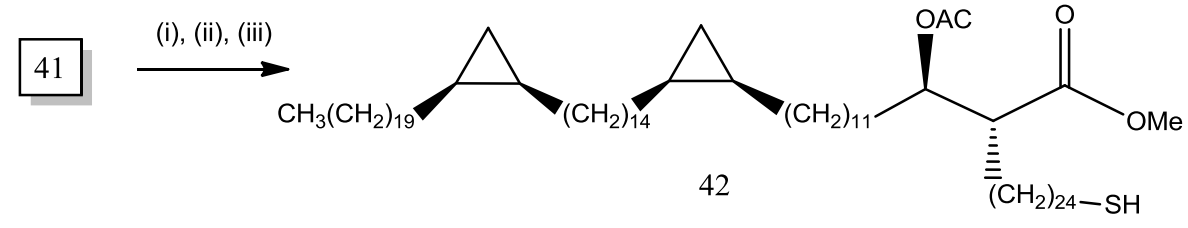

Scheme 12: (i) excess diazomethane; (ii) acetic anhydride, pyridine, toluene; (iii) DL-dithiothreitol 
Again, this was confirmed by the shift of the signal for the methylene group adjacent to sulfur for 2.67 to 2.52 in the ${ }^{1} \mathrm{H}$ NMR spectrum, the latter appearing as a quartet in chloroform solution. Compound (42) represents a thiolated example of an $\alpha$-mycolic acid with the chain lengths seen for the major homologue of the natural mixture [Al Dulayymi et al, 2003, 2005].

\section{Conclusion}

Four thiolated mycolic acids have been prepared as single diastereoisomers. Disulfide (24) was immobilized on a gold electrode substrate and the surface investigated by cyclic voltammetry (Figure 1). The binding of this molecule to gold and its application in the diagnosis of tuberculosis will be reported in full elsewhere. The CV proves the presence of an overlayer on the gold substrate that restricts charge transfer to the electrolyte solution. This is seen as a reduction of the current (the y axis). It is also significant to note that the compound was stable under $25 \mathrm{mV}$ of electrical perturbations and 20 cycles of ramping. Ozoemena et. al. suggested that different organothiolate self assembled monolayers should be investigated as platforms for electrochemical immunosensors [Ozoemena, 2009; 2010]. The thiolated MAs synthesized here provide this opportunity.

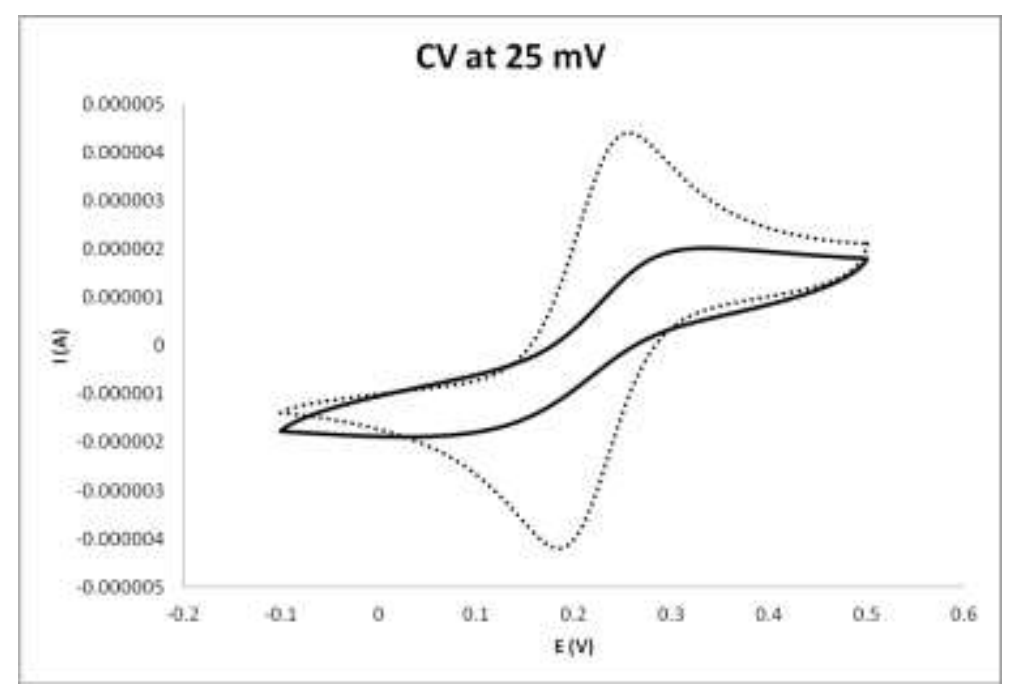

Figure 1: Cyclic voltammograms of gold (-----) and mycolic acid disulfide (24) immobilised on gold (-). The gold electrode was incubated with disulfide for 34 hours at room temperature. 


\section{Experimental section}

Chemicals used were obtained from commercial suppliers (Sigma, Aldrich, and Alfa Ayser) or prepared from them by the methods described. Solvents which were required to be dry, e.g. ether, tetrahydrofuran were dried over sodium wire and benzophenone under nitrogen, while dichloromethane and HMPA were dried over calcium hydride. All reagents and solvents used were of reagent grade unless otherwise stated. Silica gel (Merck 7736) and silica gel plates used for column chromatography and thin layer chromatography were obtained from Aldrich; separated components were detected using variously UV light, $\mathrm{I}_{2}$ and phosphomolybdic acid solution in IMS followed by charring. Anhydrous magnesium sulfate was used to dry organic solutions. Infra-red (IR) spectra were carried out on a Perkin-Elmer 1600 F.T.I.R. spectrometer as liquid films or $\mathrm{KBr}$ disc (solid). Melting points were measured using a Gallenkamp melting point apparatus. NMR spectra were carried out on a Bruker AC250 or Advance 400 spectrometer. $[\alpha]_{\mathrm{D}}$ values were recorded in $\mathrm{CHCl}_{3}$ on a POLAAR 2001 optical activity polarimeter. Mass spectra were recorded on a Bruker matrix-assisted laser desorption/ionisation-time of flight mass spectrometry (MALDI-TOF MS) values are given plus sodium to an accuracy of 2 d.p.

\section{Methyl (2R)-2-((R)-1-(tert-butyldimethylsilyloxy)-3-hydroxypropyl)-16- (tetrahydro-2H-pyran-yloxy)hexadecanoate (7)}

Lithium bis(trimethylsilyl)amide $(8.63 \mathrm{ml}, 9.15 \mathrm{mmol}, 1.06 \mathrm{M})$ was added to a stirred solution of aldehyde (5) (2.31 g, $5.86 \mathrm{mmol})$ (supplementary information) and 1phenyl-5-(12-((tetrahydro-2H-pyran-2-yloxy)dodecylsulfonyl)-1H-tetrazole (6) (3.36

$\mathrm{g}, 7.03 \mathrm{mmol}$ ) (supplementary information) in dry $\mathrm{THF}(150 \mathrm{ml})$ at $-10{ }^{\circ} \mathrm{C}$. The solution turned bright yellow/orange and was allowed to reach room temperature, stirred overnight under nitrogen, then quenched by the addition of a sat.aq. $\mathrm{NH}_{4} \mathrm{Cl}$ $(150 \mathrm{ml})$ at $-20{ }^{\circ} \mathrm{C}$, extracted with petrol/ethyl acetate $(1: 2,3 \mathrm{x} 150 \mathrm{ml})$ and the combined organic layers were dried, filtered and evaporated. Column chromatography (petrol/ethyl acetate, 20:1) gave methyl (2R)-2-((R)-3-(benzyloxy)-1-(tertbutyldimethylsilyloxy)propyl)-16-(tetrahydro-2H-pyran-2-yloxy)-hexadec-4-enoate $(2.86 \mathrm{~g}, 4.42 \mathrm{mmol}, 75 \%)$ as a colourless oil. Palladium on carbon $(10 \%, 0.5 \mathrm{~g})$ was slowly added under a stream of nitrogen to a stirred solution of the alkene $(2.56 \mathrm{~g}$, $3.96 \mathrm{mmol})$ in IMS $(50 \mathrm{ml})$ and THF $(10 \mathrm{ml})$. The flask purged of air by repeated application of vacuum followed by refilling the system with hydrogen. When the hydrogenation was complete, the mixture was filtered through a pad of Celite ${ }^{\circledR}$, which 
was washed with copious ethyl acetate and the solvent was evaporated. Column chromatography (petrol/ethyl acetate, 5:1) gave methyl $(2 R)-2-((R)-1-($ tert-butyldimethylsilyloxy)-3-hydroxypropyl)-16-(tetrahydro-2H-pyranyloxy)hexadecanoate (7) $(1.75 \mathrm{~g}, 79 \%)$ as a colourless oil, $[\alpha]_{D}^{23}-3.98\left(c 0.93, \mathrm{CHCl}_{3}\right)\left\{\right.$ Found $(\mathrm{M}+\mathrm{Na})^{+}$: 581.4215, $\mathrm{C}_{31} \mathrm{H}_{62} \mathrm{O}_{6} \mathrm{SiNa}$ requires 581.4208\}. This showed; $\delta_{\mathrm{H}}: 4.58(0.8 \mathrm{H}, \mathrm{dd}, J 2.9$, 4.4), $4.53(0.6 \mathrm{H}$, ddd, $J$ 4.7, 9.6, $11.2 \mathrm{~Hz}), 4.14(0.5 \mathrm{H}, \mathrm{m}), 4.28(1.2 \mathrm{H}, \mathrm{m}), 3.88(1 \mathrm{H}$, ddd, $J$ 3.2, 7.9, 11.4, $\left.\mathrm{CH}_{2} \mathrm{CH}(\mathrm{CH}) \mathrm{O}\right), 3.74(1.7 \mathrm{H}, \mathrm{m}), 3.68\left(1 \mathrm{H}, \mathrm{s}, \mathrm{OCH}_{3}\right), 3.51(0.7 \mathrm{H}$, m), $3.50\left(2 \mathrm{H}, \mathrm{s}, \mathrm{OCH}_{3}\right), 3.39\left(1 \mathrm{H}, \mathrm{dt}, J 6.7,9.6, \mathrm{CH}_{2} \mathrm{CH}(\mathrm{CH}) \mathrm{O}\right), 2.64(0.4 \mathrm{H}, \mathrm{ddd}, J$ 3.8, 6.9, 10.9, $\left.\mathrm{CHCH}(\mathrm{CO}) \mathrm{CH}_{2}\right), 2.31\left(0.6 \mathrm{H}\right.$, ddd, $J$ 3.2, 5.4, 8.5, $\left.\mathrm{CHCH}(\mathrm{CO}) \mathrm{CH}_{2}\right)$, $2.09(0.6 \mathrm{H}, \mathrm{m}), 1.89-2.02(1.3 \mathrm{H}, \mathrm{m}), 1.70-1.85(2.7 \mathrm{H}, \mathrm{m}), 1.58(10.4 \mathrm{H}, \mathrm{m}), 1.26$ $(20 \mathrm{H}, \mathrm{m}), 0.89\left(9 \mathrm{H}, \mathrm{s}, \mathrm{C}\left(\mathrm{CH}_{3}\right)_{3}\right.$, including smaller s at 0.88$), 0.09\left(3 \mathrm{H}, \mathrm{s}, \mathrm{CH}_{3} \mathrm{Si}\right.$, including smaller s at 0.11$)$ and $0.08\left(3 \mathrm{H}, \mathrm{s}, \mathrm{CH}_{3} \mathrm{Si}\right.$, including smaller s at 0.07$) ; \delta_{\mathrm{C}}$ : $173.1,99.8,98.9,67.7,65.9,64.7,63.1,62.4,61.9,55.0,47.6,32.8,31.7,30.8,30.5$, 29.8, 29.7, 29.63, 29.60, 29.5, 29.4, 27.2, 26.4, 26.3, 25.74, 25.68, 25.5, 19.7, 19.4, 18.0,-4.3 and -5.0; $v_{\max }\left(\right.$ film) $/ \mathrm{cm}^{-1}: 3465,2926,2854,1737$ and 1463.

\section{Methyl (2R)-2-((R)-1-(tert-butyldimethylsilyloxy)-3-oxopropyl)-16-(tetrahydro-}

\section{H-pyran-2-yloxy)hexadecanoate}

A solution of alcohol (7) $(1.73 \mathrm{~g}, 3.10 \mathrm{mmol})$ in dichloromethane $(10 \mathrm{ml})$ was added to a stirred suspension of PCC $(1.67 \mathrm{~g}, 7.75 \mathrm{mmol})$ in dichloromethane $(100 \mathrm{ml})$ at room temperature and stirred at room temperature for $3 \mathrm{hrs}$. Ethyl acetate $(50 \mathrm{ml})$ was added and the mixture was filtered through a bed of silica and the solvent was evaporated. Column chromatography (petrol/ethyl acetate, 5:2) gave methyl (2R)-2((R)-1-(tert-butyldimethylsilyloxy)-3-oxopropyl)-16-(tetrahydro-2H-pyran-2-yloxy)hexadecanoate $(1.41 \mathrm{~g}, 82 \%)$ as a colourless oil, $[\alpha]_{D}^{21}-7.04\left(c 0.98, \mathrm{CHCl}_{3}\right)$. This showed $\delta_{\mathrm{H}}: 9.18(1 \mathrm{H}, \mathrm{dd}, J 1.6,2.5 \mathrm{~Hz}), 4.58(1 \mathrm{H}, \mathrm{m}), 4.43(1 \mathrm{H}, \mathrm{m}), 3.88$ (1H, ddd, $J$ 3.3, 7.7, $11.2 \mathrm{~Hz}), 3.74(1 \mathrm{H}, \mathrm{dt}, J$ 6.9, $9.6 \mathrm{~Hz}), 3.69(3 \mathrm{H}, \mathrm{s}), 3.51(1 \mathrm{H}, \mathrm{m}), 3.39(1 \mathrm{H}$, $\mathrm{dt}, J$ 6.7, 9.6 Hz), $0.07(3 \mathrm{H}, \mathrm{s}), 2.60(3 \mathrm{H}, \mathrm{m}), 1.83(1 \mathrm{H}, \mathrm{m}), 1.72(1 \mathrm{H}, \mathrm{m}), 1.56(10 \mathrm{H}$, m), $1.28(20 \mathrm{H}, \mathrm{m}), 0.86(9 \mathrm{H}, \mathrm{s})$ and $0.08(3 \mathrm{H}, \mathrm{s}) ; \delta_{\mathrm{C}}: 201.3,174.1,98.9,68.8,67.7$, 62.4, 52.3, 51.6, 48.1, 30.8, 29.8, 29.7, 29.63, 29.56, 29.52, 29.4, 27.8, 27.0, 26.3, 25.6, 25.5, 19.7, 17.9, -4.6 and $-4.9 ; v_{\max }\left(\right.$ film) $/ \mathrm{cm}^{-1}: 2927,2855,1735$ and 1464. This aldehyde was used immediately in the next step. 


\section{Methyl (2R,3R)-3-(tert-butyldimethylsilyloxy)-2-(14-hydroxytetradecyl) heneicosanoate (8)}

(i) Lithium bis(trimethylsilyl)amide (3.68 ml, $3.90 \mathrm{mmol}, 1.06 \mathrm{M})$ was added to a stirred solution of methyl (2R)-2-((R)-1-(tert-butyldimethylsilyloxy)-3-oxopropyl)16-(tetra-hydro-2 $H$-pyran-2-yloxy)hexadecanoate $(1.39 \mathrm{~g}, 2.50 \mathrm{mmol})$ and 5(hexadecyl-sulfonyl)-1-phenyl-1H-tetrazole $(1.30 \mathrm{~g}, 3.00 \mathrm{mmol})$ in dry THF (25 ml) at $-10{ }^{\circ} \mathrm{C}$. The solution turned bright yellow/orange and was allowed to reach room temperature, and stirred overnight under nitrogen. It was quenched by addition of a sat.aq. $\mathrm{NH}_{4} \mathrm{Cl}(100 \mathrm{ml})$ at $-20{ }^{\circ} \mathrm{C}$ then extracted with petrol/ethyl acetate $(1: 2,3 \times 150$ $\mathrm{ml}$ ) and the combined organic layers were dried, filtered and evaporated. Column chromatography (petrol/ethyl acetate, 15:1) gave methyl $(2 R, 3 R)$-3-(tert-butyldimethylsilyloxy)-2-(14-(tetrahydro-2H-pyran-2-yloxy)tetradecyl)heincos-5-enoate $(1.43 \mathrm{~g} 1.87 \mathrm{mmol}, 75 \%)$ as a colourless oil.

(ii) Palladium on carbon $(10 \%, 0.3 \mathrm{~g})$ was added to a stirred solution of olefin (1.40 $\mathrm{g}, 1.83 \mathrm{mmol})$ in IMS $(20 \mathrm{ml})$ and THF $(10 \mathrm{ml})$ and the mixture was hydrogenated as above then filtered through a pad of Celite ${ }^{\circledR}$, which was washed with copious ethyl acetate. The solvent was evaporated Column chromatography (petrol/ethyl acetate, 10:1) gave methyl (2R,3R)-3-(tert-butyldimethylsilyloxy)-2-(14-(tetrahydro- $2 \mathrm{H}$ pyran-2-yloxy)tetradecyl)henicosanoate $(1.30 \mathrm{~g}, 92 \%)$ as a colourless oil, $[\alpha]_{D}^{21.3}-$ $4.15\left(c\right.$ 0.92, $\left.\mathrm{CHCl}_{3}\right) \quad$ Found $(\mathrm{M}+\mathrm{Na})^{+}:$789.6748, $\mathrm{C}_{47} \mathrm{H}_{94} \mathrm{O}_{5} \mathrm{SiNa}$ requires $789.6763\}$. This showed $\delta_{\mathrm{H}}: 4.58(1 \mathrm{H}, \mathrm{dd}, J 3.0,4.3 \mathrm{~Hz}), 3.91(2 \mathrm{H}, \mathrm{m}), 3.74(1 \mathrm{H}, \mathrm{dt}, J$ 7.0, $9.6 \mathrm{~Hz}), 3.66(3 \mathrm{H}, \mathrm{s}), 3.51(1 \mathrm{H}, \mathrm{m}), 3.39$ (1H, dt, $J$ 6.7, $9.6 \mathrm{~Hz}), 2.53$ (1H, ddd, $J$ 3.7, 7.2, $11.0 \mathrm{~Hz}), 1.57-1.83(16 \mathrm{H}, \mathrm{m}), 1.26(50 \mathrm{H}, \mathrm{m}), 0.88(3 \mathrm{H}, \mathrm{t}, J 6.9 \mathrm{~Hz}), 0.87$ $(9 \mathrm{H}, \mathrm{s}), 0.05(3 \mathrm{H}, \mathrm{s})$ and $0.02(3 \mathrm{H}, \mathrm{s}) ; \delta_{\mathrm{C}}: 175.2,98.9,76.6,73.2,67.7,62.3,51.6$, 51.2, 33.7, 32.8, 31.9, 30.8, 29.83, 29.77, 29.71, 29.66, 29.60, 29.52, 29.46, 29.37, $27.9,27.5,26.3,25.8,25.5,23.7,22.7,19.7,18.0,14.1,-4.4$ and $-4.9 ; v_{\max }($ film $) / \mathrm{cm}^{-1}$ : $2924,2853,1740$ and 1464.

(iii) Pyridinium $p$-toluenesulfonate $(40 \mathrm{mg}, 1.58 \mathrm{mmol}$ ) was added with stirring to methyl (2R,3R)-3-(tert-butyldimethylsilyloxy)-2-(14-(tetrahydro-2H-pyran-2-yloxy)tetra-decyl)-henicosanoate $(485 \mathrm{mg}, 0.633 \mathrm{mmol})$ in THF $(15 \mathrm{ml})$, methanol $(3 \mathrm{ml})$ and water $(1 \mathrm{ml})$ at room temperature and stirred overnight. Sat.aq. $\mathrm{NaHCO}_{3}(10 \mathrm{ml})$ and petrol/ethyl acetate $(1: 1,10 \mathrm{ml})$ were added. The mixture was extracted with 
petrol/ethyl acetate $(1: 1,3 \times 25 \mathrm{ml})$ and the combined organic layers were washed with brine $(20 \mathrm{ml})$, dried, filtered and evaporated. Column chromatography (petrol/ ethyl acetate, 5:1) gave methyl (2R,3R)-3-(tert-butyldimethylsilyloxy)-2-(14-hydroxytetradecyl)heneicosanoate $(\mathbf{8})(352 \mathrm{mg}, 82 \%)$ as a colourless oil, $[\alpha]_{D}^{22.4}-3.74(c 0.95$, $\left.\mathrm{CHCl}_{3}\right)$ \{Found $(\mathrm{M}+\mathrm{Na})^{+}:$705.6205, $\mathrm{C}_{42} \mathrm{H}_{86} \mathrm{O}_{4} \mathrm{SiNa}$ requires 705.6188\}. This showed $\delta_{\mathrm{H}}: 3.91(1 \mathrm{H}, \mathrm{dt}, J$ 4.7, $6.8 \mathrm{~Hz}), 3.66(3 \mathrm{H}, \mathrm{s}), 3.65(2 \mathrm{H}, \mathrm{t}, J 6.6 \mathrm{~Hz}), 2.53(1 \mathrm{H}$, ddd, J 3.8, 7.2, $11.0 \mathrm{~Hz}), 1.53(9 \mathrm{H}$, br m), $1.26(52 \mathrm{H}, \mathrm{m}), 0.88(3 \mathrm{H}, \mathrm{t}, J 7.1), 0.87(9 \mathrm{H}$, s), $0.05(3 \mathrm{H}, \mathrm{s})$ and $0.02(3 \mathrm{H}, \mathrm{s}) ; \delta_{\mathrm{C}}: 175.2,73.2,63.1,51.6,33.7,32.8,31.9,29.8$, 29.71, 29.66, 29.63, 29.58, 29.4, 29.3, 27.9, 27.5, 25.8, 25.7, 23.7, 22.7, 18.0, 14.1, 4.3 and $-4.9 ; v_{\max }($ film $) / \mathrm{cm}^{-1}: 3357,2924,2853,1739$ and 1462.

\section{Methyl (2R,3R)-3-(tert-butyldimethylsilyloxy)-2-(14-(tosyloxy)tetradecyl) heneicosanoate}

A solution of alcohol (8) $(256 \mathrm{mg}, 0.376 \mathrm{mmol})$ and triethylamine $(1 \mathrm{ml})$ in dry dichloromethane $(10 \mathrm{ml})$ was cooled to $-20{ }^{\circ} \mathrm{C}$ under $\mathrm{N}_{2}(\mathrm{~g})$ and stirred for 30 minutes. $p$-Toluenesulfonyl chloride $(86 \mathrm{mg}, 0.451 \mathrm{mmol}$ ) was added in one portion. The solution was kept in the refrigerator overnight and then the solvent was evaporated. Column chromatography (petrol/ethyl acetate, $5: 1)$ gave methyl $(2 R, 3 R)$ 3-(tert-butyldimethylsilyloxy)-2-(14-(tosyloxy)tetradecyl)heneicosanoate (233 mg, 74 $\%)$ as a colourless oil, $[\alpha]_{D}^{20.7}-0.82\left(c 0.94, \mathrm{CHCl}_{3}\right)\left\{\right.$ Found $(\mathrm{M}+\mathrm{Na})^{+}:$859.33, $\mathrm{C}_{49} \mathrm{H}_{92} \mathrm{O}_{6} \mathrm{SSiNa}$ requires: 859.63 \}. This showed $\delta_{\mathrm{H}}: 7.80(2 \mathrm{H}, \mathrm{d}, J 8.5 \mathrm{~Hz}), 7.36(2 \mathrm{H}$, $\mathrm{d}, J 7.9 \mathrm{~Hz}), 4.02(2 \mathrm{H}, \mathrm{t}, J 6.5 \mathrm{~Hz}), 3.91(1 \mathrm{H}, \mathrm{dt}, J 4.7,6.9 \mathrm{~Hz}), 3.66(3 \mathrm{H}, \mathrm{s}), 2.52(1 \mathrm{H}$, ddd, $J$ 3.7, 7.2, $10.8 \mathrm{~Hz}), 2.46(3 \mathrm{H}, \mathrm{s}), 1.63(8 \mathrm{H}, \mathrm{m}), 1.26(52 \mathrm{H}, \mathrm{m}), 0.88$ (3H, t, J 6.9 $\mathrm{Hz}), 0.87(9 \mathrm{H}, \mathrm{s}), 0.05(3 \mathrm{H}, \mathrm{s})$ and $0.02(3 \mathrm{H}, \mathrm{s}) \delta_{\mathrm{C}}: 175.1,144.6,133.3,129.8,127.9$, 73.2, 70.7, 60.4, 51.6, 51.2, 33.7, 31.9, 29.8, 29.70, 29.67, 29.64, 29.61, 29.59, 29.51, $29.47,29.40,29.36,28.9,28.8,27.9,27.5,25.8,25.3,23.7,22.7,21.6,21.0,18.0$, 14.2, 14.1, -4.3 and $-4.9 ; v_{\max }($ film $) / \mathrm{cm}^{-1}: 2925,2854,1739,1598,1464$. 


\section{Methyl (2R,3R)-2-(14-(acetylthio)tetradecyl)-3-(tert-butyldimethylsilyloxy) heneicosanoate (9)}

Methyl (2R,3R)-3-(tert-butyldimethylsilyloxy)-2-(14-(tosyloxy)tetradecyl)heneicosanoate $(231 \mathrm{mg}, 0.276 \mathrm{mmol})$ and potassium thioacetate $(126 \mathrm{mg}, 0.111 \mathrm{mmol})$ in acetone $(13 \mathrm{ml})$ and THF $(5 \mathrm{ml})$ were stirred at room temperature for $4 \mathrm{hrs}$, then the solvent was removed. Column chromatography (petrol/ethyl acetate, 5:1) gave methyl (2R,3R)-2-(14-(acetylthio)tetradecyl)-3-(tert-butyldimethylsilyloxy)henicosanoate (9) $(154 \mathrm{mg}, 75 \%)$ as a colourless oil, $[\alpha]_{D}^{24.2}-1.73\left(c 0.47, \mathrm{CHCl}_{3}\right)\left\{\right.$ Found $(\mathrm{M}+\mathrm{Na})^{+}$: 763.5978, $\mathrm{C}_{44} \mathrm{H}_{88} \mathrm{O}_{4} \mathrm{SSiNa}$ requires 763.6070$\}$. This showed $\delta_{\mathrm{H}}: 3.91(1 \mathrm{H}, \mathrm{dt}, J$ 4.7, $6.9 \mathrm{~Hz}), 3.66(3 \mathrm{H}, \mathrm{s}), 2.87$ (2H, t, J 7.4 Hz), 2.53 (1H, ddd, J 3.8, 7.1, $10.9 \mathrm{~Hz}), 2.32$ $(3 \mathrm{H}, \mathrm{s}), 1.26-1.55(60 \mathrm{H}, \mathrm{m}), 0.88(3 \mathrm{H}, \mathrm{t}, J 7 \mathrm{~Hz}), 0.86(9 \mathrm{H}, \mathrm{s}), 0.05(3 \mathrm{H}, \mathrm{s})$ and 0.02 $(3 \mathrm{H}, \mathrm{s}) ; \delta_{\mathrm{C}}: 196.0,175.1,73.2,51.6,33.7,31.9,30.6,29.8,29.64$ (br), 29.58, 29.50, $29.48,29.45,29.37,29.2,29.1,28.8,27.9,27.5,25.8,23.7,22.7,18.0,14.1,-4.3$ and $-4.9 ; v_{\max }($ film $) / \mathrm{cm}^{-1}: 2926,2847,1737,1695$ and 1460 .

\section{Methyl (2R,3R)-2-(14-bromotetradecyl)-3-(tert-butyldimethylsilyloxy) heneicosanoate (10)}

This is described in the supplementary information.

\section{Methyl (2R,3R)-2-(14-(acetylthio)tetradecyl)-3-hydroxyheneicosanoate (11)}

Thioacetate (9) $(287 \mathrm{mg}, 0.388 \mathrm{mmol})$ was dissolved in dry THF (6 ml) in a dry polyethylene vial under $\mathrm{N}_{2}(\mathrm{~g})$ at $0{ }^{\circ} \mathrm{C}$. Pyridine $(0.2 \mathrm{ml}, 2.48 \mathrm{mmol})$ and HF.pyridine ( $1.5 \mathrm{ml}, \mathrm{mmol})$ were added and the mixture stirred at $45{ }^{\circ} \mathrm{C}$ overnight, then added slowly to a sat.aq. $\mathrm{NaHCO}_{3}(20 \mathrm{ml})$. The solution was extracted with petrol/ethyl acetate $(1: 1,3 \times 20 \mathrm{ml})$ and the combined organic layers were dried, filtered and evaporated. Column chromatography (petrol/ethyl acetate, 10:1) gave methyl (2R,3R)-2-(14-(acetylthio)tetradecyl)-3-hydroxyhenicosanoate (11) (199 mg, $84 \%)$ as a white solid, m.p. $66-68{ }^{\circ} \mathrm{C},[\alpha]_{D}^{249}+5.87\left(c\right.$ 0.59, $\left.\mathrm{CHCl}_{3}\right)\left\{\right.$ Found $(\mathrm{M}+\mathrm{Na})^{+}$: 649.5224, $\mathrm{C}_{38} \mathrm{H}_{74} \mathrm{O}_{4} \mathrm{SiNa}$ requires 649.5200$\}$. This showed $\delta_{\mathrm{H}}: 3.71(3 \mathrm{H}, \mathrm{s}), 3.66(1 \mathrm{H}$, m), $2.86(2 \mathrm{H}, \mathrm{t}, J 7.4 \mathrm{~Hz}), 2.44(1 \mathrm{H}, \mathrm{dt}, J 5.3,9.3, \mathrm{~Hz}), 2.32(3 \mathrm{H}, \mathrm{s}), 2.09(1 \mathrm{H}, \mathrm{br} \mathrm{s}$, 
$\mathrm{OH}), 1.56(4 \mathrm{H}, \mathrm{m}), 1.45(4 \mathrm{H}, \mathrm{m}), 1.26(52 \mathrm{H}, \mathrm{m})$ and $0.88(3 \mathrm{H}, \mathrm{t}, J 6.9 \mathrm{~Hz}) ; \delta_{\mathrm{C}}: 196.0$, 176.2, 72.3, 51.5, 51.0, 35.7, 31.9, 30.6, 29.70 (br), 29.65, 29.62, 29.57, 29.50, 29.47, 29.42, 29.3, 29.2, 29.1, 28.8, 27.4, 25.7, 22.7 and 14.1; $v_{\max }\left(\right.$ film) $/ \mathrm{cm}^{-1}: 3407,2921$, 2852, 1732,1688 and 1465.

\section{$\left(2 R, 2^{\prime} R, 3 R, 3\right.$ ' $\left.R\right)$-2,2'-Disulfanediylbis(tetradecane-14,1-diyl))bis(3-hydroxy}

\section{heneicosanoic acid (13)}

Lithium hydroxide (4 mol.eq, $10.7 \mathrm{mg}, 0.255 \mathrm{mmol}$ ) was added to methyl $(2 R, 3 R)-2-$ (14-(acetylthio)tetradecyl)-3-hydroxyhenicosanoate (11) $(40 \mathrm{mg}, 0.064 \mathrm{mmol})$ in a mixture of THF $(4 \mathrm{ml})$, water $(0.4 \mathrm{ml})$ and methanol $(0.4 \mathrm{ml})$ and stirred at $45{ }^{\circ} \mathrm{C}$ overnight. The reaction was diluted by addition of petrol/ethyl acetate $(1: 1,10 \mathrm{ml})$ and brought to $\mathrm{pH} 1$ by dropwise addition of dil. $\mathrm{HCl}$. The product was extracted with petrol/ethyl acetate $(1: 1,10 \mathrm{ml})$ and the combined organic extracts were dried and evaporated. Column chromatography (petrol/ethyl acetate, $5: 2)$ gave $\left(2 R, 2^{\prime} R, 3 R, 3^{\prime} R\right)$ 2,2'-disulfanediylbis(tetradecane-14,1-diyl))bis(3-hydroxyheneicosanoic acid (13) $(9.3 \mathrm{mg}, 26 \%)$ as a white solid, $[\alpha]_{D}^{20}-0.15\left(c \quad 0.23, \mathrm{CHCl}_{3}\right)$ \{Found $(\mathrm{M}-\mathrm{H})^{+}$: 1138.3447, $\mathrm{C}_{70} \mathrm{H}_{137} \mathrm{O}_{6} \mathrm{~S}_{2}$ requires 1137.9857\}. This showed $\delta_{\mathrm{H}}: 3.70(1 \mathrm{H}, \mathrm{m}), 2.70$ $(2 \mathrm{H}, \mathrm{t}, J 7.3 \mathrm{~Hz}), 2.47(1 \mathrm{H}, \mathrm{dt}, J 4.9,9.6 \mathrm{~Hz}), 1.68(4 \mathrm{H}, \mathrm{m}), 1.52(3 \mathrm{H}, \mathrm{m}), 1.26(56 \mathrm{H}$, m) and $0.89(3 \mathrm{H}, \mathrm{t}, J 6.8 \mathrm{~Hz}) ; \delta_{\mathrm{C}}: 180.7,72.3,51.0,39.5,35.5,31.9,29.70,29.67$, 29.61, 29.56, 29.52, 29.43, 29.36, 29.2, 29.1, 28.9, 28.4, 27.4, 25.7, 22.7, 22.6 and $14.1 ; v_{\max }\left(\mathrm{CHCl}_{3}\right) / \mathrm{cm}^{-1}: 3451,2916,2850,1682$ and 1470.

\section{2,2-Dimethylpropanoic acid 22-(1-phenyl-1H-tetrazole-5-ylsulfonyl)docosyl ester} (15)

(i) 1-Phenyl-1H-tetrazole-5-thiol (4.4 g, $24.6 \mathrm{mmol}), 2,2$-dimethylpropanoic acid (22-bromo)docosyl ester (11.0 g, $22.4 \mathrm{mmol})$ (supplementary information) and anhydrous potassium carbonate $(6.81 \mathrm{~g}, 49.3 \mathrm{mmol})$ were vigorously stirred in acetone $(250 \mathrm{ml})$ for $18 \mathrm{hrs}$ at room temperature. Water $(500 \mathrm{ml})$ was added and the product was extracted with dichloromethane $(1 \times 200 \mathrm{ml}, 2$ x $100 \mathrm{ml})$. The combined organic layers were washed with brine $(2 \times 200 \mathrm{ml})$, dried and evaporated. Column chromatography eluting with petrol/ethyl acetate (10:1) gave a semi-solid 2,2dimethylpropanoic acid 22-(1-phenyl-1H-tetrazole-5-ylsulfanyl)docosyl ester (13.0 
g, $84 \%)\left\{\right.$ Found $(\mathrm{M}+\mathrm{H})^{+}: 587.4344, \mathrm{C}_{34} \mathrm{H}_{59} \mathrm{O}_{2} \mathrm{~N}_{4} \mathrm{~S}$ requires: 587.4353$\} ; \delta_{\mathrm{H}}(500$ $\left.\mathrm{MHz}, \mathrm{CDCl}_{3}\right): 7.58-7.51(5 \mathrm{H}, \mathrm{m}), 4.03(2 \mathrm{H}, \mathrm{t}, J 6.65 \mathrm{~Hz}), 3.38(2 \mathrm{H}, \mathrm{t}, J 7.55 \mathrm{~Hz})$, $1.84(2 \mathrm{H}$, pent, $J 6.5 \mathrm{~Hz}), 1.60(2 \mathrm{H}$, pent, $J 6.3 \mathrm{~Hz}), 1.44-1.39(2 \mathrm{H}, \mathrm{m}), 1.33-1.21$

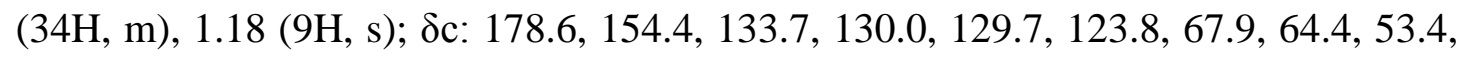
$38.7,33.3,30.8,29.5,29.48,29.45,29.4,29.2$, 29.0, 28.6, 28.55, 27.1, 25.8, 25.6; v max: $2925,2853,1728,1597,1500,1462,1397,1283,1156 \mathrm{~cm}^{-1}$.

(ii) Ammonium molybdate (VI) tetrahydrate (13.70 g, $11.09 \mathrm{mmol})$ in $35 \% \mathrm{H}_{2} \mathrm{O}_{2}(50$ $\mathrm{ml}$ ), prepared and cooled in an ice bath was added to a stirred solution of the above sulfide $(13 \mathrm{~g}, 22.18 \mathrm{mmol})$ in THF $(50 \mathrm{ml})$ and $\mathrm{IMS}(100 \mathrm{ml})$ at $10{ }^{\circ} \mathrm{C}$. The mixture was stirred at room temperature for $2 \mathrm{hrs}$. Further ammonium molybdate (VI) tetrahydrate $(6.85 \mathrm{~g}, 5.5 \mathrm{mmol})$ in $35 \% \mathrm{H}_{2} \mathrm{O}_{2}(25 \mathrm{ml})$ was added and the mixture was stirred at room temperature for $18 \mathrm{hrs}$, then poured into water $(1 \mathrm{~L})$ and extracted with dichloromethane $(1 \times 250 \mathrm{ml}, 3 \times 150 \mathrm{ml})$. The combined organic phases were washed with water $(500 \mathrm{ml})$, dried and the evaporated. The product was purified by column chromatography eluting petrol/ethyl acetate (5:1, then $1: 1)$ to give a white solid, 2,2dimethylpropanoic acid 22-(1-phenyl-1H-tetrazole-5-ylsulfonyl)docosyl ester (15) (12.4 g, 90\%), mp 41-42 ${ }^{\circ} \mathrm{C}$ \{Found $(\mathrm{M}+\mathrm{Na})^{+}: 641.4071, \mathrm{C}_{34} \mathrm{H}_{58} \mathrm{O}_{2} \mathrm{SNa}$ requires: 641.4076); $\delta_{\mathrm{H}}:\left(500 \mathrm{MHz}, \mathrm{CDCl}_{3}\right) ; 7.61-7.60(2 \mathrm{H}, \mathrm{m}), 7.59-7.58(3 \mathrm{H}, \mathrm{m}), 4.04(2 \mathrm{H}$, t, $J 6.65 \mathrm{~Hz}), 3.73(2 \mathrm{H}, \mathrm{t}, J 7.4 \mathrm{~Hz}), 1.95-1.92(2 \mathrm{H}, \mathrm{m}), 1.61(2 \mathrm{H}$, pent, $J 6.95 \mathrm{~Hz})$, $1.50(2 \mathrm{H}$, pent, $J 6.65 \mathrm{~Hz}), 1.37-1.22(34 \mathrm{H}, \mathrm{m}), 1.19(9 \mathrm{H}, \mathrm{s}) ; \delta \mathrm{c}: 178.6,153.5,133.0$, 131.4, 129.7, 125.0, 64.4, 60.3, 56.0, 38.7, 29.7, 29.6, 29.5, 29.47, 29.4, 29.2, 29.15, 28.9, 28.6, 28.1, 27.2, 25.9, 21.9, 21.0; $v_{\max }$ : 2918, 2850, 1725, 1617, 1497, 1473, $1342,1285,1155,824 \mathrm{~cm}^{-1}$.

\section{(R)-2-[(-(R)-1-(tert-Butyldimethylsilanyloxy)-3-hydroxypropyl]-26-(2,2-}

\section{dimethylpropionyloxy)-hexacosanoic acid methyl ester (16)}

(i) Lithium bis(trimethylsilyl)amide $(14.6 \mathrm{ml}, 15.48 \mathrm{mmol})$ was added to a stirred solution of (2R,3R)-5-benzyloxy-3-(tert-butyldimethylsilanyloxy)-2-(oxoethyl)pentanoic acid methyl ester (5) (3.7 g, $9.38 \mathrm{mmol})$ and ester (15) (6.39 g, $10.32 \mathrm{mmol})$ in dry THF $(100 \mathrm{ml})$ under nitrogen at $-10^{\circ} \mathrm{C}$. The reaction turned bright yellow and was left to reach r.t. and stirred for $1 \mathrm{hr}$ under nitrogen, then quenched with sat.aq. $\mathrm{NH}_{4} \mathrm{Cl}$. The product was extracted with petrol/ethyl acetate $(20: 1,3 \times 150 \mathrm{ml})$, dried, filtered and evaporated. Column chromotography over silica eluting with petrol/ethyl acetate 
(20:1) gave a colourless oil, methyl $(E / Z)((R)-2-[(R)-1$-(tert-butyldimethylsilanyloxy)3-benzyloxypropyl]-26-(2,2-dimethylpropionyloxy)hexacos-3-enoic acid methyl ester $(5 \mathrm{~g}, 67 \%)$, in ratio $(2: 1)$.

(ii) Palladium on carbon $10 \%$ ( $1 \mathrm{~g}$ ) was added to a stirred solution of the alkene in IMS (50 ml) and THF (50 ml) under hydrogen. Hydrogenation was carried out for 2 days. The mixture was filtered over a bed of celite and the solvent was evaporated. Column chromatography eluting with petrol/ ethyl acetate (5:1) gave a white solid, $(R)$-2-[(-(R)-1-(tert-butyldimethylsilanyloxy)-3-hydroxypropyl]-26-(2,2dimethylpropionyloxy)hexacosanoic acid methyl ester (16) (3.0 g, 68\%), m.p 37-39 ${ }^{\circ} \mathrm{C}$, $[\alpha]_{D}^{23}=-8.89\left(\mathrm{c} 1.54, \mathrm{CHCl}_{3}\right)\left\{\right.$ Found $[\mathrm{M}+\mathrm{Na}]^{+}:$721.5739. $\mathrm{C}_{41} \mathrm{H}_{82} \mathrm{O}_{6}$ SiNa requires 721.5773\}; $\delta_{\mathrm{H}}\left(500 \mathrm{MHz}, \mathrm{CDCl}_{3}\right): 4.04(2 \mathrm{H}, \mathrm{t}, J 6.5 \mathrm{~Hz}), 3.81-3.70(1 \mathrm{H}, \mathrm{m}), 3.78-3.7$ $(2 \mathrm{H}, \mathrm{m}), 3.67(3 \mathrm{H}, \mathrm{s}), 2.64(1 \mathrm{H}, \mathrm{ddd}, J 3.75,6.9,10.7 \mathrm{~Hz}), 1.83-1.73(2 \mathrm{H}, \mathrm{m}), 1.64-$ $1.58(4 \mathrm{H}, \mathrm{m}), 1.29-1.18(52 \mathrm{H}, \mathrm{m}$, including s at $\delta 1.20), 0.88(9 \mathrm{H}, \mathrm{s}), 0.11(3 \mathrm{H}, \mathrm{s})$, $0.07(3 \mathrm{H}, \mathrm{s}) ; \delta \mathrm{c}: 178.7,174.7,72.1,64.5,59.5,51.6,51.4,38.7,35.2,29.7,29.6$, 29.55, 29.5, 29.4, 29.2, 28.6, 27.9, 27.2, 25.9, 25.7, 22.6, 22.3, 21.0, 17.9, 14.19, -4.5, $-5.0 ; v_{\max }: 3521,2925,2854,1731,1463,1285,1255,1163,1092,837 \mathrm{~cm}^{-1}$.

\section{(R)-2-[(R)-1-(tert-Butyldimethylsilanyloxy)-3-oxopropy]-26-(2,2-dimethyl- propionyloxy)-hexacosanoic acid methyl ester (17)}

(R)-2-[(-(R)-1-(tert-Butyldimethylsilanyloxy)-3-hydroxypropyl]-26-(2,2-dimethylpropionyloxy)-hexacosanoic acid methyl ester (3 g, $4.46 \mathrm{mmol})$ in dichloromethane $(20 \mathrm{ml})$ was added to a stirred suspension of PCC $(2.40 \mathrm{~g}, 11.15 \mathrm{mmol})$ in dichloromethane $(130 \mathrm{ml})$ at room temperature. The mixture was stirring vigorously for $2 \mathrm{hrs}$, then poured into petrol/ethyl acetate $(10: 1,300 \mathrm{ml})$, filtered through a bed of silica and celite then washed well with petrol/ethyl acetate (10:1) and evaporated. Column chromatography eluting with petrol/ethyl acetate (10:1) gave colourless oil, $(R)$-2-[(R)-1-(tert-butyldimethylsilanyloxy)-3-oxo-propy]-26-(2,2-dimethylpropionyloxy)hexacosanoic acid methyl ester (17) $(2.46 \mathrm{~g}, 82.2 \%),[\alpha]_{D}^{19}=-4.16$ (c 0.96, $\left.\mathrm{CH}_{3} \mathrm{Cl}\right)$ \{Found $[\mathrm{M}+\mathrm{Na}]^{+}:$719.5565, $\mathrm{C}_{41} \mathrm{H}_{80} \mathrm{O}_{6} \mathrm{SiNa}$ requires: 719.5616$\}, \delta_{\mathrm{H}}:(500$ $\left.\mathrm{MHz}, \mathrm{CDCl}_{3}\right): 9.82(1 \mathrm{H}$, br.s), $4.44-4.41(1 \mathrm{H}, \mathrm{m}), 4.04(2 \mathrm{H}, \mathrm{t}, J 6.5 \mathrm{~Hz}), 3.68(3 \mathrm{H}$, s), 2.67-2.57 $(3 \mathrm{H}, \mathrm{m}), 1.61(2 \mathrm{H}$, pent, $J 6.5 \mathrm{~Hz}), 1.25-1.19(53 \mathrm{H}, \mathrm{m}$, including s at $\delta$ 1.19 ), $0.85(9 \mathrm{H}, \mathrm{s}), 0.074(3 \mathrm{H}, \mathrm{s}) 0.072(3 \mathrm{H}, \mathrm{s})$; $\delta \mathrm{c}: 201.3,178.6,174.0,68.8,64.5$, 52.3, 51.5, 48.1, 38.7, 31.6, 29.7, 29.6, 29.55, 29.5, 29.4, 29.2, 29.0, 28.6, 27.7, 27.2, 
$27.0,25.9,25.6,22.6,17.9,14.1,-4.6,-4.9 ; v_{\max }: 2927,2856,1731,1463,1364$, $1285,1162,1005,837,777 \mathrm{~cm}^{-1}$.

\section{Methyl (R)-2-((R)-1-(tert-butyldimethylsilyoxy)-11-hydroxyundecyl)-26- (pivaloyloxy)hexacosanoate (18)}

(i) Lithium bis(trimethylsilyl)amide $(7.33 \mathrm{ml}, 8.4 \mathrm{mmol})$ was added with stirring to ester (17) (3.14 g, $4.5 \mathrm{mmol})$ and 1-phenyl-1H-tetrazol-5-(8-(tetrahydro-2H-pyran-2yloxy)octsulfonyl)- $1 H$-tetrazole $(2.47,5.8 \mathrm{mmol})$ in dry THF $(80 \mathrm{ml})$ under nitrogen at $-10{ }^{\circ} \mathrm{C}$. The reaction turned bright yellow and was left to reach room temperature and stirred for $1 \mathrm{hr}$ under nitrogen, then quenched with sat.aq. $\mathrm{NH}_{4} \mathrm{Cl}(20 \mathrm{ml})$. The product was extracted with petrol/ethyl acetate (10:1) ( 3 x $150 \mathrm{ml}$ ). The combined organic layers were dried and evaporated. Column chromotography of the residue eluting with petrol/ethyl acetate (20:1) gave a colourless oil, $(R)$-methyl-2-((Z/E)-1(tert-butyldimethylsilyoxy)-11-(tetrahydro-2H-pyran-2-yloxy)-undec-3-enyl)-26(pivaloyloxy)hexacosanoate in ratio (2.5:1) (3.4 g, 85\%).

(ii) Pyridinium p-toluenesulfonate $(0.47 \mathrm{~g}, 1.90 \mathrm{mmol})$ was added to a stirred solution of the above alkenes $(3.4 \mathrm{~g}, 3.80 \mathrm{mmol})$ in $\mathrm{MeOH}-\mathrm{THF}(30 \mathrm{ml}: 70 \mathrm{ml})$ at $50{ }^{\circ} \mathrm{C}$ for 3 hrs. The solvent was evaporated and the residue was treated with sat.aq. $\mathrm{NaHCO}_{3}(30$ $\mathrm{ml})$ and petrol/ethyl acetate $(10: 1,70 \mathrm{ml})$. The aqueous layer was re-extracted with petrol/ethyl acetate $(10: 1,3 \times 50 \mathrm{ml})$. The combined organic layers were dried and evaporated. Chromatography eluting with petrol/ethyl acetate (15:1 then 5:1) gave $(R)$-methyl-2-((Z/E)-1-(tert-butyldimethyl-silyoxy)-11-hydroxyundec-3-enyl)-26(pivaloyloxy)hexacosanoate as a colourless oil $(2.7 \mathrm{~g}, 88 \%)$.

(iii) Palladium on carbon $10 \%(0.7 \mathrm{~g})$ was added to a stirred solution of the above alcohol (2.7 g, $3.33 \mathrm{mmol})$ in IMS/THF (2:1, 40:20 ml) under hydrogen. Hydrogenation was carried out for $1 \mathrm{hr}$, then the mixture was filtered over a bed of celite and the solvent was evaporated. Column chromatography eluting with petrol/ethyl acetate (5:1) gave methyl $(R)-$-2-((R)-1-(tert-butyldimethylsilyoxy)-11-hydroxyundecyl)-26-

(pivaloyloxy)hexacosanote (18) as a semi-solid $(2.4 \mathrm{~g}, 88 \%),[\alpha]_{D}^{22}=-3.69$ (c 1.19, $\left.\mathrm{CHCl}_{3}\right) \quad\left\{\right.$ Found $(\mathrm{M}+\mathrm{Na})^{+}:$833.7025; $\mathrm{C}_{49} \mathrm{H}_{98} \mathrm{O}_{6} \mathrm{SiNa}$ requires: 833.7030\}; $\delta_{\mathrm{H}}$ : $\left(500 \mathrm{MHz}, \mathrm{CDCl}_{3}\right) ; 4.03(2 \mathrm{H}, \mathrm{t}, J 7.3 \mathrm{~Hz}), 3.90-3.87(1 \mathrm{H}, \mathrm{m}), 3.64(3 \mathrm{H}, \mathrm{s}), 3.62(2 \mathrm{H}, \mathrm{t}$, $J$ 6.5 Hz), $2.51(1 \mathrm{H}$, ddd, $J 3.75,7.25,10.7 \mathrm{~Hz}), 1.61-1.50(6 \mathrm{H}, \mathrm{m}), 1.44-1.18(68 \mathrm{H}$, $\mathrm{m}$, including s at $\delta 1.18), 0.85(9 \mathrm{H}, \mathrm{s}), 0.03(3 \mathrm{H}, \mathrm{s}), 0.009(3 \mathrm{H}, \mathrm{s}) ; \delta \mathrm{c}: 178.6,175.1$, 
$73.2,64.4,63.0,51.5,51.2,38.7,33.6,32.8,29.8,29.7,29.6,29.5,29.48,29.4,29.2$, 28.6, 27.8, 27.2, 25.9, 25.8, 25.7, 23.7, 21.0, 17.9, 14.2, -4.4, -5.0; $v_{\max }: 3344,2927$, $2854,1732,1655,1546,1463,1284,1253,1157,1034,836,775 \mathrm{~cm}^{-1}$.

\section{(R)-2-[(R)-1-tert-Butyldimethylsilanyloxy)-11-oxopropyl]-26-(2,2-dimethyl-} propionyloxy)hexacosanoic acid methyl ester (19)

The ester $18(1.2 \mathrm{~g}, 1.47 \mathrm{mmol})$ in dichloromethane $(20 \mathrm{ml})$ was added to a stirred suspension of pyridinium chlorochromate $(0.79 \mathrm{~g}, 3.69 \mathrm{mmol})$ in dichloromethane $(50$ $\mathrm{ml}$ ) at room temperature, stirred vigorously for $2 \mathrm{hrs}$, then poured into petrol/ethyl acetate $(10: 1,150 \mathrm{ml})$ and filtered through a bed of silica and celite, washed with petrol/ethyl acetate $(50 \mathrm{ml})$ and evaporated. Chromatography eluting with petrol/ethyl acetate (10:1) gave a colourless oil, $(R)-2-[(R)$-1-tert-butyldimethylsilanyloxy)-11oxopropyl]-26-(2,2-dimethylpropionyloxy)hexacosanoic acid methyl ester (19) (1 g, $84 \%),[\alpha]_{D}^{20}=-3.98\left(\mathrm{c} 0.94, \mathrm{CHCl}_{3}\right) ; \delta_{\mathrm{H}}:\left(500 \mathrm{MHz}, \mathrm{CDCl}_{3}\right): 9.77(1 \mathrm{H}, \mathrm{t}, J 3.75 \mathrm{~Hz})$, $4.03(2 \mathrm{H}, \mathrm{t}, J 5.05 \mathrm{~Hz}), 3.92-3.89(1 \mathrm{H}, \mathrm{m}), 3.66(3 \mathrm{H}, \mathrm{s}), 2.54-2.50(1 \mathrm{H}, \mathrm{m}), 2.44-2.40$ $(2 \mathrm{H}, \mathrm{m}), 1.62(2 \mathrm{H}$, pent, $J 6.9 \mathrm{~Hz}), 1.43-1.39(1 \mathrm{H}, \mathrm{m}), 1.28-1.14(68 \mathrm{H}, \mathrm{m}$, including s at $\delta 1.20), 0.86(9 \mathrm{H}, \mathrm{s}), 0.04(3 \mathrm{H}, \mathrm{s}), 0.02(3 \mathrm{H}, \mathrm{s}) ; \delta \mathrm{c}: 202.8,178.6,175.1,73.2,64.5$, 51.6, 51.2, 43.9, 38.7, 33.7, 29.8, 29.7, 29.6, 29.58, 29.55, 29.5, 29.48, 29.4, 29.3, $29.3,29.2,29.2,28.6,27.8,27.5,27.2,25.9,23.8,22.1,18.0,-4.4,-4.9$; $v_{\max }: 2924$, $2852,1734,1709,1607,1494,1402,1107,1050,824 \mathrm{~cm}^{-1}$.

\section{Methyl $(R)-2-((R)-1-(t e r t-b u t y l d i m e t h y l s i l o x y)-18-(1 R, 2 S)-2-(17 S, 18 S)-17-$}

methoxy-18-methylhexatriacontyl)cyclopropropyl)octadecyl)-26-hydroxyhexacosanoate (21)

(i) Lithium bis(trimethylsilyl)amide $(2.17 \mathrm{ml}, 2.30 \mathrm{mmol}, 1.06 \mathrm{M})$ was added dropwise to a stirred solution of ester (19) $(0.97 \mathrm{~g}, 1.19 \mathrm{mmol})$ and tetrazole (20) $(1.23 \mathrm{~g}, 1.37 \mathrm{mmol})$ in dry THF $(50 \mathrm{ml})$ at $-10{ }^{\circ} \mathrm{C}$ under nitrogen. The reaction turned bright yellow and was left to reach room temperature and stirred for $1 \mathrm{hr}$, then quenched with sat.aq. $\mathrm{NH}_{4} \mathrm{Cl}(30 \mathrm{ml})$. The product was extracted with petrol/ethyl acetate (10:1) (3 x $150 \mathrm{ml})$ dried and evaporated. Column chromotography eluting with petrol/ethyl acetate (20:1) gave a colourless oil, methyl $(E / Z)-(R)-2-((R)-1-($ tertbutyldimethylsiloxy)-18-(1R,2S)-2-(17S,18S)-17-methoxy-18-methylhexatriacontyl)- 
cyclopropyl)octadec-11-enyl)-26-(pivaloyloxy)hexacosanoate $(1.2 \mathrm{~g}, 71 \%)$ in ratio $2: 1$.

(ii) Dipotassium azodicarboxylate (2 g, $10.3 \mathrm{mmol}$ ) was added to a stirred solution of the above alkenes $(1.2 \mathrm{~g}, 0.81 \mathrm{mmol})$ in THF $(30 \mathrm{ml})$ and methanol $(7 \mathrm{ml})$ at $5{ }^{\circ} \mathrm{C}$. A solution of glacial acetic acid $(3 \mathrm{ml})$ and THF $(3 \mathrm{ml})$ was added dropwise at $5^{\circ} \mathrm{C}$ over a period two days. Further dipotassium azodicarboxylate $(1.5 \mathrm{~g})$ then glacial acetic acid $(2 \mathrm{ml})$ in THF $(2 \mathrm{ml})$ were added and stirred overnight. This mixture was poured slowly into sat. aq. $\mathrm{NaHCO}_{3}(20 \mathrm{ml})$. The product was extracted with petrol/ethyl acetate $(1: 1,3 \times 80 \mathrm{ml})$. The combined organic layers were washed with water $(50$ $\mathrm{ml})$, dried and evaporated. Column chromatography eluting with petrol/ethyl acetate (15:1) gave methyl $(R)-2-((R)-1-($ tert-butyldimethylsiloxy)-18-(1R,2S)-2-(17S,18S)17-methoxy-18-methylhexatriacontyl)-cyclopropyl)octadecyl)-26-(pivaloyloxy)hexacosanoate as a thick colourless oil, $(0.94 \mathrm{~g}, 78 \%),[\alpha]_{D}^{23}=-5.60$ (c $\left.1.07 \mathrm{~g}, \mathrm{CHCl}_{3}\right)$ \{Found $[\mathrm{M}+\mathrm{Na}]^{+}:$1504.4639; $\mathrm{C}_{97} \mathrm{H}_{192} \mathrm{O}_{6} \mathrm{NaSi}$ requires: 1504.4380$\} ; \delta_{\mathrm{H}}:(500 \mathrm{MHz}$, $\left.\mathrm{CDCl}_{3}\right): 4.06(2 \mathrm{H}, \mathrm{t}, J 6.6 \mathrm{~Hz}), 3.92-3.89(1 \mathrm{H}, \mathrm{m}), 3.66(3 \mathrm{H}, \mathrm{s}), 3.34(3 \mathrm{H}, \mathrm{s}), 2.97-$ $2.94(1 \mathrm{H}, \mathrm{m}), 2.53(1 \mathrm{H}, \mathrm{ddd}, J 3.8,7.25,11.00 \mathrm{~Hz}), 1.64-1.48(8 \mathrm{H}, \mathrm{m}), 1.37-1.13$ $(145 \mathrm{H}, \mathrm{m}$, including $\mathrm{s}$ at 1.20$), 0.90-0.82(18 \mathrm{H}, \mathrm{m}$, including $\mathrm{s}$ at $\delta 0.86), 0.66-0.64$ (2H, br. m), $0.56(1 \mathrm{H}, \mathrm{dt}, J 3.75,7.9 \mathrm{~Hz}), 0.04(3 \mathrm{H}, \mathrm{s}), 0.02(3 \mathrm{H}, \mathrm{m}),-0.32(1 \mathrm{H}, \mathrm{q}, J$, $5.35 \mathrm{~Hz})$; $\delta \mathrm{c}: 178.3,175.1,85.4,73.2$, 64.5, 57.7, 51.6, 51.2, 38.7, 35.3, 33.7, 32.4, $31.9,30.5,30.2$, 30.0, 29.8, 29.7, 29.6, 29.5, 29.4, 29.35, 29.2 , 28.9, 28.6, 27.8, 27.6, $27.2,26.2,25.8,23.7,22.68,18.0,15.8,14.9,14.1,10.9,-4.4,-4.9 ; v_{\max }: 2923,2853$, $1732,1464,1156,1099,836 \mathrm{~cm}^{-1}$.

(iii) The above product $(0.94 \mathrm{~g}, 0.63 \mathrm{mmol})$ in THF $(3 \mathrm{ml})$ was added to a stirred solution of potassium hydroxide $(0.0 .53 \mathrm{~g}, 9.45 \mathrm{mmol})$ in THF (15 ml), methanol (15 $\mathrm{ml})$ and water $(1.5 \mathrm{ml})$. The mixture was heated at $70{ }^{\circ} \mathrm{C}$ for $3 \mathrm{~h}$, then quenched with water $(10 \mathrm{ml})$ and extracted with petrol/ethyl acetate $(10: 1,3 \times 25 \mathrm{ml})$. The combined organic extracts were dried, filtered and evaporated. Column chromotography eluting with petrol/ethyl acetate (10:1) gave methyl $(R)-2-((R)$-1-(tert-butyldimethylsiloxy)18-(1R,2S)-2-(17S,18S)-17-methoxy-18-methylhexatriacontyl)cyclopropyl)octa-

decyl)-26-hydroxyhexacosanoate $(\mathbf{2 1})(0.62 \mathrm{~g}, 70 \%)$, as a thick colourless oil, $[\alpha]_{D}^{23}=$ -4.62 (c $0.67, \mathrm{CHCl}_{3}$ ) \{Found $[\mathrm{M}+\mathrm{Na}]^{+}:$1420.37; $\mathrm{C}_{92} \mathrm{H}_{184} \mathrm{O}_{5} \mathrm{NaSi}$ requires: $1420.3805\} ; \delta_{\mathrm{H}}:\left(500 \mathrm{MHz}, \mathrm{CDCl}_{3}\right): 3.92-3.89(1 \mathrm{H}, \mathrm{m}), 3.66(3 \mathrm{H}, \mathrm{s}), 3.64(2 \mathrm{H}, \mathrm{t}, J$ $6.95 \mathrm{~Hz}), 2.97-2.94(1 \mathrm{H}, \mathrm{m}), 2.55(1 \mathrm{H}, \mathrm{ddd}, J 3.8,7.25,11.00 \mathrm{~Hz}), 1.59-1.54(8 \mathrm{H}, \mathrm{m})$, 
1.37-1.14 (140H, m), 0.90-0.84 (18H, m, including s at $\delta 0.86), 0.64(2 \mathrm{H}$, br. $\mathrm{m}), 0.56$ $(1 \mathrm{H}, \mathrm{dt}, J 4.1,8.15 \mathrm{~Hz}), 0.04(3 \mathrm{H}, \mathrm{s}), 0.02(3 \mathrm{H}, \mathrm{s}),-0.32(1 \mathrm{H}, \mathrm{q}, J, 5.00 \mathrm{~Hz}) ; \delta \mathrm{c}$ : 175.1, 85.4, 73.2, 63.1, 57.7, 51.6, 51.2, 35.3, 33.7, 32.8, 32.4, 31.9, 30.5, 30.2, 30.0, 29.9, 29.8, 29.6, 29.4, 29.35, 28.7, 27.8, 27.6, 27.5, 26.2 , 25.8, 23.7, 22.7, 17.9, 15.8, $14.9,14.1,10.9,-4.4,-4.9 ; v_{\max }: 3371,2921,2852,1741,1466,1097,836 \mathrm{~cm}^{-1}$.

Methyl $(R)-2-((R)-1-($ tert-butyldimethylsiloxy)-18-(1S,2R)-2-(17R,18R)-17methoxy-18-methylhexatriacontyl)cyclopropropyl)octadecyl)-26-hydroxy hexacosanoate (26)

(i) Lithium bis(trimethylsilyl)amide $(2.17 \mathrm{ml}, 2.30 \mathrm{mmol}, 1.06 \mathrm{M})$ was added dropwise to a stirred solution of ester (19) $(1.00 \mathrm{~g}, 1.33 \mathrm{mmol})$ and tetrazole (25) $(1.37 \mathrm{~g}, 1.53 \mathrm{mmol})$ [Al Dulayymi, 2007] in dry THF $(50 \mathrm{ml})$ at $-10{ }^{\circ} \mathrm{C}$. The reaction turned bright yellow and was left to reach r.t. and stirred for $1 \mathrm{hr}$ under nitrogen then quenched with sat.aq. $\mathrm{NH}_{4} \mathrm{Cl}(25 \mathrm{ml})$. The product was extracted with petrol/ethyl acetate (10:1, 3 x $150 \mathrm{ml})$, dried, filtered and evaporated. Column chromotography eluting with petrol/ethyl acetate $(20: 1)$ gave a colourless oil, methyl $(E / Z)(R)-2-((R)-$ 1-(tert-butyldimethylsiloxy)-18-(1S,2R)-2-(17R,18R)-17-methoxy-18-methylhexatriacontyl)cyclopropyl)octadec-11-enyl)-26-(pivaloyloxy)hexacosanoate (1.6 g, 89\%) in ratio (2:1). Dipotassium azodicarboxylate $(2 \mathrm{~g}, 10.3 \mathrm{mmol})$ was added to a stirred solution of the alkene mixture $(1.6 \mathrm{~g}, 1.10 \mathrm{mmol})$ in THF (30 ml) and methanol (7 ml) at $5{ }^{\circ} \mathrm{C}$. A solution of glacial acetic acid $(3 \mathrm{ml})$ and THF $(3 \mathrm{ml})$ was prepared and added dropwise at $5{ }^{\circ} \mathrm{C}$ over two days. Further dipotassium azodicarboxylate $(1.5 \mathrm{~g})$ and glacial acetic acid $(2 \mathrm{ml})$ were added and stirred overnight. This mixture was added slowly to sat.aq. $\mathrm{NaHCO}_{3}$, extracted with petrol/ethyl acetate $(1: 1,3 \times 80 \mathrm{ml}$,) and the combined organic layers were washed with water $(50 \mathrm{ml})$ and the solvent was evaporated. The product was purified by column chromatography eluting with petrol/ethyl acetate (15:1) to give methyl $(R)$ - 2-((R)-1-(tert-butyldimethylsiloxy)-18$(1 S, 2 R)-2-(17 R, 18 R)-17$-methoxy-18-methyl-hexatriacontyl)cyclopropyl)octadecyl)-

26-(pivaloyloxy)hexacosanoate as a thick colourless oil $(1.5 \mathrm{~g}, 93 \%),[\alpha]_{D}^{22}=+2.75$ (c 0.87, $\mathrm{CHCl}_{3}$ ) \{Found $[\mathrm{M}+\mathrm{Na}]^{+}:$1504.4309; $\mathrm{C}_{97} \mathrm{H}_{192} \mathrm{O}_{6} \mathrm{SiNa}$ requires 1504.4580$\}, \delta_{\mathrm{H}}$ : $\left(500 \mathrm{MHz}, \mathrm{CDCl}_{3}\right): 4.05(2 \mathrm{H}, \mathrm{t}, J 6.65 \mathrm{~Hz}), 3.95-3.89(1 \mathrm{H}, \mathrm{m}), 3.66(3 \mathrm{H}, \mathrm{s}), 3.34(3 \mathrm{H}$, s), 2.96-2.94 (1H, m), $2.53(1 \mathrm{H}$, ddd, $J 3.8,7.25,11.05 \mathrm{~Hz}), 1.64-1.59(4 \mathrm{H}, \mathrm{m}), 1.37-$ $1.22(143 \mathrm{H}, \mathrm{m}), 1.21(9 \mathrm{H}, \mathrm{s}), 0.90-0.84(15 \mathrm{H}, \mathrm{m}$, including $\mathrm{s}$ at $\delta 0.87), 0.66-0.63$ 
(2H, m), 0.56 (1H, br.dt, J, 4.1, $8.2 \mathrm{~Hz}), 0.04(3 \mathrm{H}, \mathrm{s}), 0.02(3 \mathrm{H}, \mathrm{s}),-0.32(1 \mathrm{H}, \mathrm{q}, J$ $4.75 \mathrm{~Hz}$ ); $\delta \mathrm{c:} 178.6,175.1,85.4,73.2$, 64.5, 57.7, 51.6, 51.2, 38.7, 35.4, 33.7, 32.4, $31.9,30.5,30.2,30.0,29.9,29.8,29.7,29.6,29.5,29.4,29.36,29.2$, 28.7, 28.6, 27.8, 27.6, 27.5, 27.2, 26.2, 25.9, 25.8, 23.7, 22.7, 18.0, 15.8, 14.9,14.1, 10.9, -4.4, -4.9; v max: 2924, 2853, 1733, 1465, 1157, 1099, 836, $775 \mathrm{~cm}^{-1}$.

(ii) The above product $(1.5 \mathrm{~g}, 1.02 \mathrm{mmol})$ in THF $(3 \mathrm{ml})$ was added to a stirred solution of potassium hydroxide $(0.88 \mathrm{~g}, 15.51 \mathrm{mmol})$ in a mixture of THF $(15 \mathrm{ml})$, methanol $(15 \mathrm{ml})$ and water $(1.5 \mathrm{ml})$. The mixture was heated at $70{ }^{\circ} \mathrm{C}$. After $3 \mathrm{~h}$, it was quenched with water $(10 \mathrm{ml})$ and extracted with petrol/ethyl acetate (10:1) (3 x $25 \mathrm{ml})$. The combined organic extracts were dried, filtered and evaporated. Column chromotography eluting with petrol/ethyl acetate (10:1) gave methyl $(R)-2-((R)-1-$ (tert-butyldimetheylsiloxy)-18-(1S,2R)-2-(17R,18R)-17-methoxy-18-methylhexatriacontyl)cyclopropyl)octadecyl)-26-hydroxyhexacosanoate (26) (0.85 g, 60\%), as a thick colourless oil, $[\alpha]_{D}^{22}=+4.14\left(\right.$ c $\left.0.94, \mathrm{CHCl}_{3}\right)\left\{\right.$ Found $[\mathrm{M}+\mathrm{Na}]^{+}: 1420.3663$; $\mathrm{C}_{92} \mathrm{H}_{184} \mathrm{O}_{5} \mathrm{SiNa}$ requires 1420.3805$\}, \delta_{\mathrm{H}}:\left(500 \mathrm{MHz}, \mathrm{CDCl}_{3}\right): 3.92-3.89(1 \mathrm{H}, \mathrm{m}), 3.66$ $(3 \mathrm{H}, \mathrm{s}), 3.64(2 \mathrm{H}, \mathrm{t}, J 6.9 \mathrm{~Hz}), 3.34(3 \mathrm{H}, \mathrm{s}), 2.97-2.94(1 \mathrm{H}, \mathrm{m}), 2.53$ (1H, ddd, J 3.45, $6.95,10.7 \mathrm{~Hz}), 1.57(2 \mathrm{H}$, pent, $J 6.6 \mathrm{~Hz}), 1.39-1.15(146 \mathrm{H}, \mathrm{m}), 0.9-0.84(15 \mathrm{H}, \mathrm{m}$ including $\mathrm{s}$ at $\delta 0.86), 0.67-0.64(2 \mathrm{H}, \mathrm{m}), 0.57(1 \mathrm{H}, \mathrm{dt}, J 4.1,8.2 \mathrm{~Hz}), 0.05(3 \mathrm{H}, \mathrm{s})$, $0.02(3 \mathrm{H}, \mathrm{s})-0.32(1 \mathrm{H}, \mathrm{q}, J 5.05 \mathrm{~Hz})$; $\delta \mathrm{c}: 175.1,85.5,73.2,63.1,57.7,51.6,51.2$, 35.4, 33.7, 32.8, 32.4, 31.9, 30.5, 30.2, 30.0, 29.9, 29.8, 29.6, 29.4, 29.35, 28.7, 27.8, $27.6,27.5,26.2,25.8,23.7,22.7,18.0,15.8,14.8,14.1,10.9,-4.4,-4.9 ; v_{\max }: 3450$, $2923,2853,1741,1464,1361,1254,1099,836,720 \mathrm{~cm}^{-1}$.

\section{Methyl $(R)-26-($ acetylthio)-2-((R)-1-(tert-butyldimethylsiloxy)-18-(1R,2S)-2- (17S,18S)-17-methoxy-18-methylhexatriacontyl)cyclopropyl) octadecyl) hexacosanoate $(22)$}

(i) Methyl $(R)-2-((R)-1-($ tert-butyldimethylsiloxy)-18-(1R,2S)-2-(17S, 18S)-17methoxy-18-methylhexatriacontyl)cyclopropyl)octadecyl)-26-hydroxyhexacosanoate (21) $(0.61 \mathrm{~g}, 0.43 \mathrm{mmol})$ and triethylamine $(3 \mathrm{ml})$ in dry dichloromethane $(25 \mathrm{ml})$ was cooled to $-20{ }^{\circ} \mathrm{C}$ under nitrogen and stirred for 30 minutes, followed by the addition of toluene sulfonylchloride $(0.108 \mathrm{~g}, 0.56 \mathrm{mmol})$ in one portion. The mixture was kept in a refrigerator for $16 \mathrm{~h}$, then the solvent was evaporated. Column chromatography with petrol/ethyl acetate (10:1) gave methyl $(R)$-2-((R)-1-(tert-butyldimethylsiloxy)-18- 
(1R,2S)-2-(17S,18S)-17-methoxy-18-methylhexatriacontyl)-cyclopropyl)octadecyl)26-(tosyloxy)hexacosanoate $(0.5 \mathrm{~g}, 75 \%)$, as a colourless oil, $[\alpha]_{D}^{20}=-7.22$ (c 0.83 , $\mathrm{CHCl}_{3}$ ) \{Found $[\mathrm{M}+\mathrm{Na}]^{+}:$1574.3777; $\mathrm{C}_{99} \mathrm{H}_{190} \mathrm{O}_{7} \mathrm{NaSiS}$ requires: 1574.3894$\} ; \delta_{\mathrm{H}}$ $\left(500 \mathrm{MHz}, \mathrm{CDCl}_{3}\right): 7.79(2 \mathrm{H}, \mathrm{d}, J 8.2 \mathrm{~Hz}), 7.34(2 \mathrm{H}, \mathrm{d}, J 7.9 \mathrm{~Hz}), 4.02(2 \mathrm{H}, \mathrm{t}, J 6.3$ Hz), 3.92-3.89 (1H, m), 3.65 (3H, s), 3.34 (3H, s), 2.97-2.94 (1H, m), 2.53 (1H, ddd, $J$ 3.8, 7.25, 11.05 Hz), $2.45(3 \mathrm{H}, \mathrm{s}), 1.64-1.60(2 \mathrm{H}, \mathrm{m}), 1.42-1.11(142 \mathrm{H}, \mathrm{m}), 0.89-0.82$ $(18 \mathrm{H}, \mathrm{m}$, including s at $\delta 0.86), 0.65-0.64(2 \mathrm{H}$, br. $\mathrm{m}), 0.56(1 \mathrm{H}, \mathrm{dt}, J 3.4,8.12 \mathrm{~Hz})$, $0.04(3 \mathrm{H}, \mathrm{s}), 0.02(3 \mathrm{H}, \mathrm{s}),-0.32(1 \mathrm{H}, \mathrm{q}, J 5.35 \mathrm{~Hz}) ; \delta \mathrm{c}: 175.1,144.5,133.3,129.8$, $127.9,85.4,73.2$, 70.7, 57.7, 51.6, 51.2, 35.3, 33.7, 32.4, 31.9, 30.5, 30.2, 30.0, 29.9, 29.8, 29.7, 29.69, 29.6, 29.58, 29.5, 29.4, 29.38, 29.35, 28.9, 28.8, 28.7, 27.8, 27.7, 27.5, 26.1, 25.7, 25.3, 23.7, 22.7, 21.6, 19.4, 17.96, 14.9, 14.2, 10.9, -4.4, -4.9; $v_{\max }$ : 2922, 2853, 1740, 1465, 1369, 1178, 1098, $836 \mathrm{~cm}^{-1}$.

(ii) Potassium thioacetate $(0.147 \mathrm{~g}, 1.29 \mathrm{mmol})$ was added to a stirred solution of the tosylate $(0.5 \mathrm{~g}, 0.32 \mathrm{mmol})$ in dry THF $(5 \mathrm{ml})$ and acetone $(15 \mathrm{ml})$ at room temperature under nitrogen and stirred for $16 \mathrm{~h}$, then the solvent was evaporated. Column chromatography eluting with petrol/ethyl acetate (20:1) gave methyl $(R)$-26(acetylthio)-2-((R)-1-(tert-butyldimethylsiloxy)-18-(1R,2S)-2-(17S,18S)-17-methoxy18-methylhexatriacontyl)cyclopropyl)octadecyl)-hexacosanoate $22(0.35 \mathrm{~g}, 76 \%)$ as a pale yellow thick oil, $[\alpha]_{D}^{20}=-5.83\left(\right.$ c $\left.1.37, \mathrm{CHCl}_{3}\right)\left\{\right.$ Found $[\mathrm{M}+\mathrm{K}]^{+}: 1478.3724$; $\mathrm{C}_{94} \mathrm{H}_{186} \mathrm{O}_{4} \mathrm{KSSi}$ requires: 1478.3473$\} ; \delta_{\mathrm{H}}:\left(500 \mathrm{MHz}, \mathrm{CDCl}_{3}\right): 3.92-3.89(1 \mathrm{H}, \mathrm{m}), 3.66$ $(3 \mathrm{H}, \mathrm{s}), 3.34(3 \mathrm{H}, \mathrm{s}), 2.97-2.94(1 \mathrm{H}, \mathrm{m}), 2.86(2 \mathrm{H}, \mathrm{t}, J 7.25 \mathrm{~Hz}), 2.53(1 \mathrm{H}, \mathrm{ddd}, J 3.8$, 7.25, $10.75 \mathrm{~Hz}), 2.32(3 \mathrm{H}, \mathrm{s}), 1.64-1.53(8 \mathrm{H}, \mathrm{m}), 1.37-1.08$ (H, m), 0.90-0.84 (18H, $\mathrm{m}$, including s at $\delta$ 0.86), 0.65-0.64 $(2 \mathrm{H}$, br. $\mathrm{m}), 0.58-0.54(1 \mathrm{H}, \mathrm{m}), 0.04(3 \mathrm{H}, \mathrm{s}), 0.02$ $(3 \mathrm{H}, \mathrm{s}),-0.32(1 \mathrm{H}, \mathrm{q}, J, 5.00 \mathrm{~Hz}) ; \delta \mathrm{c}: 196.1,175.1,85.4,73.2,57.7,51.6,51.2,35.3$, 33.7, 32.4, 31.9, 30.6, 30.5, 30.2, 30.0, 29.9, 29.8, 29.7, 29.7, 29.6, 29.5, 29.47, 29.4, 29.1, 28.8, 27.8, 27.6, 26.2, 25.8, 23.7, 22.7, 18.0, 15.8, 14.9, 14.1, 10.9, -4.4, -4.9; v max: 2924, 2853, 1740, 1697, 1465, 1360, 1254, 1099, $836 \mathrm{~cm}^{-1}$.

\section{Methyl (R)-26-(acetylthio)-2-((R)-1-(tert-butyldimethylsiloxy)-18-(1S,2R)-2- (17R,18R)-17-methoxy-18-methylhexatriacontyl)cyclopropyl)octadecyl)}

\section{hexacosanoate (27)}

(i) Toluene sulfonylchloride $(0.143 \mathrm{~g}, 0.754 \mathrm{mmol})$ was added to a stirred solution of methyl $(R)-2-((R)-1$-(tert-butyldimethylsiloxy)-18-(1S,2R)-2-(17R,18R)-17-methoxy- 
18-methylhexatriacontyl)cyclopropyl)octadecyl)-26-hydroxyhexacosanoate $(0.81 \mathrm{~g}$, $0.58 \mathrm{mmol})$ and triethylamine $(3 \mathrm{ml})$ in dry dichloromethane $(25 \mathrm{ml})$ at $-20{ }^{\circ} \mathrm{C}$ under nitrogen. The solution was kept in a refrigerator overnight, then evaporated. Column chromatography eluting with petrol/ethyl acetate (10:1) gave methyl $(R)-2-((R)-1-$ (tert-butyldimethylsiloxy)-18-(1S,2R)-2-(17R,18R)-17-methoxy-18-methylhexatriacontyl)cyclopropyl)octadecyl)-26-(tosyloxy)-hexacosanoate (0.66 g, 74\%), as a colourless oil, $[\alpha]_{D}^{22}=+15.17\left(\right.$ c $\left.0.87, \mathrm{CHCl}_{3}\right)$ \{Found $[\mathrm{M}+\mathrm{Na}]^{+}:$1574.3817; $\mathrm{C}_{99} \mathrm{H}_{190} \mathrm{O}_{7} \mathrm{SSiNa}$ requies 1574.3894$\}, \delta_{\mathrm{H}}\left(500 \mathrm{MHz}, \mathrm{CDCl}_{3}\right): 7.80(2 \mathrm{H}, \mathrm{d}, J, 8.2 \mathrm{~Hz})$, 7.35 (2H, d, J 8.00 Hz), 4.02 (2H, t, J $6.30 \mathrm{~Hz}), 3.92-3.89$ (1H, m), 3.66 (3H, s), 3.34 $(3 \mathrm{H}, \mathrm{s}), 2.96-2.94(1 \mathrm{H}, \mathrm{m}), 2.53$ (1H, ddd, $J$ 3.8, 7.25, $10.9 \mathrm{~Hz}), 2.45(3 \mathrm{H}, \mathrm{s}), 1.63$ $(4 \mathrm{H}$, pent, $J 6.3 \mathrm{~Hz}), 1.57-1.53(2 \mathrm{H}, \mathrm{m}), 1.41-1.19(141 \mathrm{H}, \mathrm{m}), 0.90-0.83(15 \mathrm{H}, \mathrm{m}$, including s at $\delta 0.88), 0.68-0.66(2 \mathrm{H}, \mathrm{m}), 0.56(1 \mathrm{H}, \mathrm{dt}, J 4.1,8.2 \mathrm{~Hz}), 0.05(3 \mathrm{H}, \mathrm{s})$, $0.02(3 \mathrm{H}, \mathrm{s}),-0.32(1 \mathrm{H}, \mathrm{q}, J 5.05 \mathrm{~Hz})$; $\delta \mathrm{c}: 175.1,144.6,133.4,129.8,127.9,85.4$, 73.2, 70.7, 57.7, 51.6, 51.2, 41.4, 35.4, 33.7, 32.4, 31.9, 30.5, 30.2, 29.98, 29.9, 29.8, $29.71,29.7,29.6,29.5,29.45,29.4,29.36,28.9,28.8,28.7,27.8,27.6,27.5,26.2$, $25.8,25.3,23.7,22.7,21.6,19.4,18.0,14.9,14.1,10.9,-4.4,-4.9 ; v_{\max }: 2923,2852$, 1740, 1464, 1253, 1099,836, $720 \mathrm{~cm}^{-1}$.

(ii) Potassium thioacetate $(0.2 \mathrm{~g}, 1.78 \mathrm{mmol})$ was added to a stirred solution of the above tosylate $(0.66 \mathrm{~g}, 0.42 \mathrm{mmol})$ dissolved in dry THF $(5 \mathrm{ml})$ and acetone $(15 \mathrm{ml})$ at room temperature. After $16 \mathrm{~h}$, the solvent was evaporated. Column chromatography eluting with petrol/ethyl acetate (20:1) gave methyl $(R)$-26-(acetylthio)-2-((R)-1-(tertbutyldimethylsiloxy)-18-(1S,2R)-2-(17R,18R)-17-methoxy-18-methylhexatriacontyl)cyclopropyl)octadecyl)hexacosanoate $27(0.51 \mathrm{~g}, 82 \%)$ as a pale yellow thick oil, $[\alpha]_{D}^{22}=+5.28\left(\mathrm{c} 0.87, \mathrm{CHCl}_{3}\right)\left\{\right.$ Found $[\mathrm{M}+\mathrm{Na}]^{+}: 1478.3565 ; \mathrm{C}_{94} \mathrm{H}_{186} \mathrm{O}_{5} \mathrm{SSiNa}$ requies 1478.3682\}; $\delta_{\mathrm{H}}$ : $\left(500 \mathrm{MHz}, \mathrm{CDCl}_{3}\right): 3.91(1 \mathrm{H}$, br.q, J 5.0 Hz), $3.66(3 \mathrm{H}, \mathrm{s}), 3.34(3 \mathrm{H}$, s), 2.96-2.94 (1H, m), $2.86(2 \mathrm{H}, \mathrm{t}, J 7.5 \mathrm{~Hz}), 2.53(1 \mathrm{H}, \mathrm{ddd}, J 3.8,7.25,10.70 \mathrm{~Hz})$, $2.32(3 \mathrm{H}, \mathrm{s}), 1.61-1.51(6 \mathrm{H}, \mathrm{m}), 1.42-1.14(141 \mathrm{H}, \mathrm{m}), 0.89-0.83(15 \mathrm{H}, \mathrm{m}$, including $\mathrm{s}$ $\delta$ 0.87), 0.67-0.64 (2H, m), $0.56(1 \mathrm{H}, \mathrm{dt}, J 3.75,8.2 \mathrm{~Hz}), 0.04(3 \mathrm{H}, \mathrm{s}), 0.02(3 \mathrm{H}, \mathrm{s}),-$ 0.32 (1H, br.q, $J, 5.05 \mathrm{~Hz}$ ); $\delta \mathrm{c:} \mathrm{196.0,} \mathrm{175.1,} \mathrm{85.5,} \mathrm{73.2,} \mathrm{57.71,} \mathrm{51.6,} \mathrm{51.2,} \mathrm{35.4,} \mathrm{33.7,}$ 32.4, 31.9, 30.61, 30.6, 30.5, 30.2, 30.0, 29.95, 29.8, 29.7, 29.71, 29.6, 29.5, 29.49, 29.46, 29.4, 29.2, 29.1, 29.06, 28.8, 27.9, 27.6, 26.2, 25.8, 25.4, 23.8, 22.7, 19.5, 17.8, $14.9,14.1,10.9,-4.4,-4.9 ; v_{\max }: 2923,2853,1740,1465,1099,836,720 \mathrm{~cm}^{-1}$. 
Methyl $(R)-26-($ acetylthio)-2-((R)-1-hydroxy-18-(1R,2S)-2-(17S,18S)-17-methoxy18-methylhexatriacontyl)cyclopropyl)octadecyl)hexacosanote (23)

Methyl (R)-26-(acetylthio)-2-((R)-1-(tert-butyldimethylsiloxy)-18-(1R,2S)-2-(17S, 18S)-17-methoxy-18-methylhexatriacontyl)cyclopropyl)octadecyl)hexacosanoate (22) $(0.35 \mathrm{~g}, 0.24 \mathrm{mmol})$ was dissolved in dry THF $(12 \mathrm{ml})$ in a dry polyethylene vial under $\mathrm{N}_{2}$ at $0{ }^{\circ} \mathrm{C}$. Pyridine $(0.1 \mathrm{ml})$ and hydrogen fluoride-pyridine complex $(0.8 \mathrm{ml})$ were added and the mixture stirred at $45{ }^{\circ} \mathrm{C}$ over night. The mixture was added slowly to a sat.aq. $\mathrm{NaHCO}_{3}(15 \mathrm{ml})$, then extracted with petrol/ ethyl acetate $(5: 1,3 \times 50 \mathrm{ml})$ and the combined organic extracts dried, filtered and evaporated. Column chromatography (petrol/ethyl acetate, 10:1) gave $(R)$-methyl-26-(acetylthio)-2-((R)-1hydroxy-18-(1R,2S)-2-(17S,18S)-17-methoxy-18-methylhexatriacontyl)cyclopropyl)octadecyl)hexacosanoate $(\mathbf{2 3})(0.2 \mathrm{~g}, 63 \%)$ as a white solid, mp $43-45^{\circ} \mathrm{C},[\alpha]_{D}^{20}=-$ 6.02 (c $0.88, \mathrm{CHCl}_{3}$ ) \{Found $[\mathrm{M}+\mathrm{Na}]^{+}:$1364.2836; $\mathrm{C}_{88} \mathrm{H}_{172} \mathrm{O}_{5} \mathrm{NaS}$ requires: $1364.2818\} ; \delta_{\mathrm{H}}\left(500 \mathrm{MHz}, \mathrm{CDCl}_{3}\right): 3.67(3 \mathrm{H}, \mathrm{s}), 3.67-3.65(1 \mathrm{H}, \mathrm{m}), 3.34(3 \mathrm{H}, \mathrm{s})$, 2.97-2.94 (1H, m), $2.87(2 \mathrm{H}, \mathrm{t}, J 7.55 \mathrm{~Hz}), 2.45-2.40(1 \mathrm{H}, \mathrm{m}), 2.32(3 \mathrm{H}, \mathrm{s}), 1.58-1.55$ $(2 \mathrm{H}, \mathrm{m}), 1.38-1.13(143 \mathrm{H}, \mathrm{m}), 0.90-0.84(9 \mathrm{H}$, including t, $J 6.65 \mathrm{~Hz}$ and d, $J 6.95 \mathrm{~Hz})$, 0.66-0.65 (2H, br.m), $0.56(1 \mathrm{H}, \mathrm{dt}, J 3.82,7.76 \mathrm{~Hz}),-0.32(1 \mathrm{H}, \mathrm{q}, J 5.05 \mathrm{~Hz}) ; \delta \mathrm{c}$ : 196.2, 176.2, 85.4, 72.3, 57.7, 51.5, 50.9, 35.7, 35.3, 32.4, 31.9, 30.6, 30.5, 30.2, 30.0, 29.9, 29.7, 29.6, 29.57, 29.5, 29.4, 29.35, 29.2, 29.1, 28.8, 28.7, 27.6, 27.4, 26.2 , 25.7, 22.7, 15.8, 14.9, 14.1, 10.9; $v_{\max }: 3285,2917,2850,1691,1470,1167,1104,720 \mathrm{~cm}^{-}$ 1 .

$\left.R, R, R, S, R, 2 R, 2^{\prime} R\right)-26,26 '-D i s u l f a n e d i y l b i s(2-((R)-1-h y d r o x y-18-((1 R, 2 S)-2-((17 S$, 18S)-17-methoxy-18-methylhexatriacontyl)cyclopropyl)octadecyl)hexacosanoic acid) (24)

Methyl (R)-26-(acetylthio)-2-((R)-1-hydroxy-18-(1R,2S)-2-(17S,18S)-17-methoxy18-methylhexatriacontyl)cyclopropyl)octadecyl)hexacosanoate $(0.1 \mathrm{~g}, 0.074 \mathrm{mmol})$ was suspended in a $5 \%$ aq. TBAH $(20 \mathrm{ml})$ and heated to $100{ }^{\circ} \mathrm{C}$ overnight, then cooled to room temperature and acidified to $\mathrm{pH} 1$ with $1 \mathrm{M} \mathrm{HCl}$ and extracted with petrol/ethylacetate $(1: 1,3 \times 30 \mathrm{ml})$. The combined organic layers were evaporated. Column chromatography (chloroform/ methanol, 10:1) gave a white solid $(R, R, R, S$, $\left.R, 2 R, 2^{\prime} R\right)-26,26$ '-disulfanediylbis(2-((R)-1-hydroxy-18-((1R,2S)-2-((17S,18S)-17methoxy-18-methylhexatriacontyl)cyclo-propyl)octadecyl)hexacosanoic acid) 
$(25 \mathrm{mg}, 26 \%)$, m.p $60-62{ }^{\circ} \mathrm{C},[\alpha]_{D}^{23}=-2.48\left(\mathrm{c} 1.37, \mathrm{CH}_{3} \mathrm{Cl}\right), \delta_{\mathrm{H}}\left(500 \mathrm{MHz}, \mathrm{CDCl}_{3}\right)$ : 3.73-3.69 (2H, m), $3.35(6 \mathrm{H}, \mathrm{s}), 2.98-2.95(2 \mathrm{H}, \mathrm{m}), 2.69$ (4H, t, J 7.25 Hz), 2.48-2.46 $(2 \mathrm{H}, \mathrm{m}), 1.69-1.47(80 \mathrm{H}, \mathrm{m}), 1.37-1.15(218 \mathrm{H}, \mathrm{m}), 0.90-0.84(12 \mathrm{H}$, including t, $J 6.65$ $\mathrm{Hz}$ and $\mathrm{d}, J 6.95 \mathrm{~Hz}), 0.64-0.65$ (4H, br.m), 0.58-0.56 (2H, m), -0.32 (2H, q, J 4.7 $\mathrm{Hz}) ; \delta \mathrm{c}: 179.8,85.6,72.1,57.7,50.9,39.3,35.5,35.3,32.4,31.9,30.5,30.2,30.0$, 29.9, 29.72, 29.7, 29.6, 29.53, 29.5, 29.4, 29.4, 29.2, 28.7, 28.5, 27.6, 27.3, 26.2, 25.7, 22.7, 15.8, 14.9, 14.1, 10.9; $v_{\max }: 3278,291,2851,1708,1465,1376,1098,719 \mathrm{~cm}^{-1}$.

\section{$\left(R, R, R, S, R, 2 R, 2^{\prime} R\right)-26,26 '-D i s u l f a n e d i y l b i s(2-((R)-1-h y d r o x y-18-((1 S, 2 R)-2-$} ((17R,18R)-17-methoxy-18-methylhexatriacontyl)cyclopropyl)octadecyl)

\section{hexacosanoic acid) (28)}

(i) The disulfane (27) (0.45 g, $0.30 \mathrm{mmol})$ was dissolved in dry THF (12 ml) in a polyethylene vial under nitrogen at $0{ }^{\circ} \mathrm{C}$. Pyridine $(0.1 \mathrm{ml})$ and hydrogen fluoridepyridine complex $(0.8 \mathrm{ml})$ were added and the mixture was stirred at $45{ }^{\circ} \mathrm{C}$ overnight. The mixture was added slowly to sat.aq. $\mathrm{NaHCO}_{3}(15 \mathrm{ml})$. The product was extracted with petrol/ ethyl acetate $(5: 1,3 \times 50 \mathrm{ml})$ and the combined organic extracts were dried and evaporated. The product was purified by column chromatography eluting with petrol/ethyl acetate (10:1) to give $(R)$-methyl-26-(acetylthio)-2-((R)-1-hydroxy18-(1S,2R)-2-(17R,18R)-17-methoxy-18-methylhexatriacontyl)cyclopropyl)octadecyl)-hexacosanoate $(0.4 \mathrm{~g}, 97 \%)$ as a white solid, mp $40-42{ }^{\circ} \mathrm{C},[\alpha]_{D}^{23}=+6.34(\mathrm{c}$ 0.82, $\mathrm{CHCl}_{3}$ ) \{Found $[\mathrm{M}+\mathrm{Na}]^{+}: 1364.2809 ; \mathrm{C}_{88} \mathrm{H}_{172} \mathrm{O}_{5} \mathrm{SNa}$ requires 1364.2818$\} ; \delta_{\mathrm{H}}$ : $\left(500 \mathrm{MHz}, \mathrm{CDCl}_{3}\right): 3.71(3 \mathrm{H}, \mathrm{s}), 3.68-3.64(1 \mathrm{H}, \mathrm{m}), 3.34(3 \mathrm{H}, \mathrm{s}), 2.96-2.94(1 \mathrm{H}, \mathrm{m})$, $2.86(2 \mathrm{H}, \mathrm{t}, J 7.6 \mathrm{~Hz}), 2.46-2.42(1 \mathrm{H}, \mathrm{m}), 2.32(3 \mathrm{H}, \mathrm{s}), 1.73-1.69(2 \mathrm{H}, \mathrm{m}), 1.63-1.53$ $(4 \mathrm{H}, \mathrm{m}), 1.46-1,14(142 \mathrm{H}, \mathrm{m}), 0.88(3 \mathrm{H}, \mathrm{t}, J 5.65 \mathrm{~Hz}), 0.85(3 \mathrm{H}, \mathrm{d}, J 6.9 \mathrm{~Hz}), 0.66-$ 0.65 (2H, br.m), 0.56 (1H, dt, J 4.1, 8.55 Hz), -0.32 (1H, q, J 5.00 Hz); $\delta \mathrm{c:} \mathrm{196,} \mathrm{176.2,}$ 85.4, 72.3, 57.7, 51.5, 51.0, 35.7, 35.6, 32.4, 31.9, 30.6, 30.5, 30.2, 30.0, 29.97, 29.9, 29.7, 29.6, 29.6, 29.57, 29.5, 29.49, 29.47, 29.4, 29.35, 29.2, 29.1, 28.8, 28.7, 27.6, $27.4,26.2,25.7,22.7,15.8,14.9,14.1,10.9 ; v_{\max }: 3518,2920,2850,1709,1694$, $1466,1165,1098,720 \mathrm{~cm}^{-1}$.

(ii) The above ester $(0.3 \mathrm{~g}, 0.22 \mathrm{mmol})$ was suspended in $5 \%$ aq. tetrabutylammonium hydroxide $(20 \mathrm{ml})$ and heated to $100{ }^{\circ} \mathrm{C}$ overnight, then cooled to room temperature and acidified to $\mathrm{pH} 1$ with $1 \mathrm{M} \mathrm{HCl}$ and extracted with petrol/ethyl acetate (1:1, 3 x 30 $\mathrm{ml}$ ). The combined organic layers were dried and evaporated. Column 
chromatography eluting with chloroform/ methanol (10:1) gave $\left(R, R, R, S, R, 2 R, 2^{\prime} R\right)$ 26,26'-disulfanediylbis(2-((R)-1-hydroxy-18-((1S,2R)-2-((17R,18R)-17-methoxy-18methylhexatriacontyl)cyclopropyl)octa-decyl)hexacosanoic acid 28 (150 mg, $54 \%)$ as a white solid, $[\alpha]_{D}^{23}=+3.76\left(\mathrm{c} 0.85, \mathrm{CHCl}_{3}\right)$, mp 61-63 ${ }^{\circ} \mathrm{C} ; \delta_{\mathrm{H}}\left(500 \mathrm{MHz}, \mathrm{CDCl}_{3}\right)$ : 3.61-3.5 (2H, m), 3.29 (6H, s), 2.94-2.91 (2H, m), 2.63 (4H, t, J 7.25 Hz), 2.35-2.31 $(2 \mathrm{H}, \mathrm{m}), 1.62$ (4H, pent, $J 6.9 \mathrm{~Hz}), 1.42-1.05(294 \mathrm{H}, \mathrm{m}), 0.81$ (6H, t, $J 6.65 \mathrm{~Hz}), 0.79$ $(6 \mathrm{H}, \mathrm{d}, J 6.60 \mathrm{~Hz}), 0.63-058$ (4H, br.m), $0.51(2 \mathrm{H}, \mathrm{dt}, J 4.1,8.5 \mathrm{~Hz}),-0.37$ (2H, q, $J$ 5.05); $\delta \mathrm{c}: 177.8,85.6,72.0,57.5,50.9,39.1,35.6,32.3,31.8,30.4,30.1,29.8,29.76$, 29.6, 29.4, 29.38, 29.3 , 29.2 , 29.1, 28.6, 28.4, 27.38, 27.3, 26.0, 25.6, 22.5, 15.6, 14.7, $13.9,10.8 ; v_{\max }: 3280,2917,2849,1714,1470,1377,1100,719 \mathrm{~cm}^{-1}$.

\section{Methyl $\quad(R)-2-((R)-1$-acetoxy-18- $((1 S, 2 R)-2-((17 R, 18 R)-17-m e t h o x y-18-m e t h y l-$}

\section{hexatriacontyl)cyclopropyl)octadecyl)-26-mercaptohexacosanoate (29)}

(i) Excess diazomethane in ether was added to the acid (28) and stirred for $30 \mathrm{~min}$. The solvent was evaporated to give dimethyl $\left(R, R, R, S, R, 2 R, 2^{\prime} R\right)-26,26$ 'disulfanediylbis(2-((R)-1-hydroxy-18-((1S,2R)-2-((17R,18R)-17-methoxy-18-methylhexatriacontyl)-cyclopropyl)octadecyl)hexacosanoate, which showed $\delta_{\mathrm{H}}$ : $(400 \mathrm{MHz}$, $\left.\mathrm{CDCl}_{3}\right): 3.70(6 \mathrm{H}, \mathrm{s}), 3.67-3.64(2 \mathrm{H}, \mathrm{m}), 3.34(6 \mathrm{H}, \mathrm{s}), 2.97-2.95(2 \mathrm{H}, \mathrm{m}), 2.68(4 \mathrm{H}, \mathrm{t}$, $J 7.28 \mathrm{~Hz}), 2.46-2.41(2 \mathrm{H}, \mathrm{m}), 1.72-1.13(296 \mathrm{H}, \mathrm{m}), 0.88(6 \mathrm{H}, \mathrm{t}, J 6.56 \mathrm{~Hz}), 0.85(6 \mathrm{H}$, d, $J 6.88 \mathrm{~Hz}), 0.68-0.65(4 \mathrm{H}, \mathrm{m}), 0.57(2 \mathrm{H}, \mathrm{dt}, J 3.76,7.92 \mathrm{~Hz}),-0.32(2 \mathrm{H}$, br.q, $J$ $4.76 \mathrm{~Hz}) ; \delta_{\mathrm{c}}: 176.3,85.4,72.3,57.7,51.5,50.9,39.2,35.7,35.3,32.4,31.9,30.5$, 30.2, 30.0, 29.9, 29.7, 29.6, 29.57, 29.5, 29.4, 29.36, 29.3, 29.2 28.7, 28.5, 27.6, 27.4, $26.2,25.7,22.7,18.4,15.8,15.7,14.9,14.1,10.9$. The product was used for next step without purification.

(ii) Acetic anhydride $(0.3 \mathrm{ml})$ and pyridine $(0.3 \mathrm{ml})$ were added to a stirred solution of the alcohol in toluene $(0.3 \mathrm{ml})$. The mixture was stirred for $18 \mathrm{hrs}$ then the solvent was evaporated under reduced pressure to give dimethyl $\left(R, R, R, S, R, 2 R, 2^{\prime} R\right)-26,26$ 'disulfanediylbis(2-((R)-1-acetoxy-18-((1S,2R)-2-((17R,18R)-17-methoxy-18-methylhexatriacontyl)-cyclopropyl)octadecyl)hexacosa-noate, which showed $\delta_{\mathrm{H}}$ : $(400 \mathrm{MHz}$, $\left.\mathrm{CDCl}_{3}\right)$ : 5.10-5.06 (2H, m), $3.68(6 \mathrm{H}, \mathrm{s}), 3.34(6 \mathrm{H}, \mathrm{s}), 2.97-2.96(2 \mathrm{H}, \mathrm{m}), 2.68(4 \mathrm{H}, \mathrm{t}, J$ $7.28 \mathrm{~Hz}), 2.62$ (2H, ddd, J 4.3, 7.0, $10.64 \mathrm{~Hz}), 2.03(6 \mathrm{H}, \mathrm{s}), 1.71-1.13(294 \mathrm{H}, \mathrm{m}), 0.88$ $(6 \mathrm{H}, \mathrm{t}, J 6.40 \mathrm{~Hz}), 0.85(6 \mathrm{H}, \mathrm{d}, J 6.88 \mathrm{~Hz}), 0.68-0.66(4 \mathrm{H}, \mathrm{m}), 0.57(2 \mathrm{H}, \mathrm{dt}, J 3.92$, $7.92 \mathrm{~Hz}),-0.32(2 \mathrm{H}$, br.q, $J 4.92 \mathrm{~Hz}) ; \delta_{\mathrm{c:}} 173.7,170.4,85.5,74.1,57.7,51.5,49.6$, 
39.2, 35.3, 32.4, 31.9, 31.7, 30.6, 30.1, 30.0, 29.9, 29.7, 29.5, 29.4, 29.2, 29.1, 29.0, $28.7,28.5,28.1,27.6,27.5,26.2,25.0,22.7,21.0,20.6,15.8,14.9,14.1,10.9$. The product was used for next step without purification.

(iii) DL-Dithiothreitol (100 mg) was added with stirring to the above ester in chloroform $(1 \mathrm{ml})$ followed by the addition of one drop of triethylamine under nitrogen. The flask was covered with aluminium foil. The mixture was stirred for 48 hrs at room temperature. The solvent was evaporated and the product was purified by column chromatography eluting with petrol/ethyl acetate (10:1) to give methyl $(R)-2-$ $((R)-1$-acetoxy-18-((1S,2R)-2-((17R,18R)-17-methoxy-18-methylhexatriacontyl)cyclopropyl)octadecyl)-26-mercaptohexacosanoate (29) how much?, \{MALDI Found $[\mathrm{M}+\mathrm{Na}]^{+}: 1364.1 ; \mathrm{C}_{88} \mathrm{H}_{172} \mathrm{O}_{5} \mathrm{SNa}$ requires 1364.3$\}$ which showed $\delta_{\mathrm{H}}(400 \mathrm{MHz}$, $\left.\mathrm{CDCl}_{3}\right)$ : 5.13-5.06 (1H, m), $3.68(3 \mathrm{H}, \mathrm{s}), 3.34(3 \mathrm{H}, \mathrm{s}), 2.97-2.94(1 \mathrm{H}, \mathrm{m}), 2.62(1 \mathrm{H}$, ddd, $J 4.36,6.88,10.88 \mathrm{~Hz}), 2.53(2 \mathrm{H}, \mathrm{q}, J 7.52 \mathrm{~Hz}), 2.03(3 \mathrm{H}, \mathrm{s}), 1.17-1.13(149 \mathrm{H}$, $\mathrm{m}), 0.83(3 \mathrm{H}, \mathrm{t}, J 6.52 \mathrm{~Hz}), 0.81(3 \mathrm{H}, \mathrm{d}, J 7.4 \mathrm{~Hz}), 0.68-0.64(2 \mathrm{H}, \mathrm{m}), 0.57(1 \mathrm{H}, \mathrm{dt}, J$ 3.76, $8.04 \mathrm{~Hz}),-.032(1 \mathrm{H}$, br.q, $J 5.16 \mathrm{~Hz})$; $\delta_{\mathrm{c}:} 173.7,170.4,85.4,74.1,57.7,51.6$, 49.6, 35.3, 34.1, 32.3, 31.9, 31.7, 30.5, 30.2, 30.0, 29.9, 29.7, 29.6, 29.57, 29.5, 29.47, 29.44, 29.4, 29.36, 29.1, 28.7, 28.4, 28.1, 27.6, 27.5, 26.2, 25.0, 24.7, 22.7, 22.3, 21.0, $15.8,14.9,14.1,10.9 ; v_{\max }: 2921,2851,1746,1609,1493,1452 \mathrm{~cm}^{-1}$.

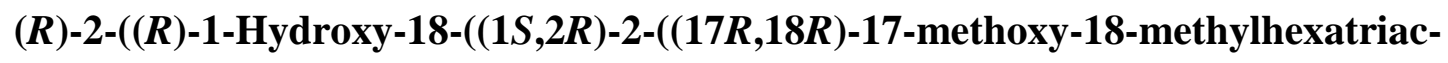 ontyl)cyclopropyl)octadecyl)-26-mercaptohexacosanoic acid (30)}

DL-Dithiothreitol $(150 \mathrm{mg})$ was added to a stirred solution of $\left(R, R, R, S, R, 2 R, 2^{\prime} R\right)$ 26,26'-disulfanediylbis(2-((R)-1-hydroxy-18-((1S,2R)-2-((17R,18R)-17-methoxy-18methylhexatriacontyl)cyclopropyl)octadecyl)hexacosanoic acid) (28) (10 mg) in chloroform $(1.5 \mathrm{ml})$ followed by the addition of one drop of triethylamine under nitrogen. The flask was covered with aluminium foil and the mixture was stirred for $48 \mathrm{hrs}$ at room temperature. The reaction was quenched with 5 drops of dil. $\mathrm{HCl}(5 \%)$ and water $(5 \mathrm{ml})$. The product was extracted with $\mathrm{CHCl}_{3}(3 \mathrm{x} 10 \mathrm{ml})$ and the combined organic layers were washed with brine solution, dried and evaporated to give a residue which was purified by column chromatography eluting with chloroform/methanol $(10: 1)$ to give $(R)-2-((R)-1$-hydroxy-18-((1S,2R)-2-((17R,18R)17-methoxy-18-methylhexatriacontyl)-cyclopropyl)octadecyl)-26-mercaptohexa- 
cosanoic acid (30) (6.5 mg) \{MALDI Found $[\mathrm{M}+\mathrm{Na}]^{+}: 1308.7 ; \mathrm{C}_{85} \mathrm{H}_{168} \mathrm{O}_{4} \mathrm{SNa}$ requires 1308.3 \} which showed $\delta_{\mathrm{H}}:\left(400 \mathrm{MHz}, \mathrm{CDCl}_{3}\right): 3.74-3.69(1 \mathrm{H}, \mathrm{m}), 3.35(3 \mathrm{H}$, s), 2.99-2.96 (1H, m), $2.52(2 \mathrm{H}, \mathrm{q}, J 7.52 \mathrm{~Hz}), 2.49-2.39(1 \mathrm{H}, \mathrm{m}), 1.74-1.11(150 \mathrm{H}$, m), $0.88(3 \mathrm{H}, \mathrm{t}, J 6.52 \mathrm{~Hz}), 0.85(3 \mathrm{H}, \mathrm{d}, J 6.92 \mathrm{~Hz}), 0.68-0.65(2 \mathrm{H}, \mathrm{m}), 0.57$ (1H, dt, $J$ 3.88, 7.76 Hz), -0.32 (1H, br.q, $J 5.24 \mathrm{~Hz})$; $\delta_{\mathrm{c}}$ : 178.0, 85.6, 72.1, 57.7, 50.7, 35.6, $35.3,34.1,32.4,31.9,30.5,30.2,30.0,29.9,29.7,29.6,29.5,29.4,29.36,29.1$, $28.7,28.4,27.6,27.3,26.2,25.7,24.7,22.7,15.8,14.9,14.1,10.9$. When the sample was shaken with $\mathrm{D}_{2} \mathrm{O}$, the quartet at $\delta 2.52$ became a triplet with $J 7.16 \mathrm{~Hz}$.

\section{Methyl $(R)-2-((R)-1$-acetoxy-18- $((1 R, 2 S)-2-((17 S, 18 S)-17-m e t h o x y-18-m e t h y l-$ hexatriacontyl)cyclopropyl)octadecyl)-26-mercaptohexacosanoate (31)}

Excess diazomethane in ether was added to $\left(R, R, R, S, R, 2 R, 2^{\prime} R\right)$-26,26'-disulfanediylbis(2-((R)-1-hydroxy-18-((1R,2S)-2-((17S,18S)-17-methoxy-18-methylhexatriacontyl)cyclopropyl)octadecyl)hexacosanoic acid) (24) and stirred for $30 \mathrm{~min}$. The solvent was evaporated to give dimethyl $\left(R, R, R, S, R, 2 R, 2^{\prime} R\right)$-26,26'-disulfanediylbis(2$((R)-1$-hydroxy-18-((1R,2S)-2-((17S,18S)-17-methoxy-18methylhexatriacontyl)cyclopropyl)-octadecyl)hexacosanoate), which showed an identical spectrum to the product from (28) and diazomethane described above. The product was used for next step without purification. Acetic anhydride $(0.3 \mathrm{ml})$ and pyridine $(0.3 \mathrm{ml})$ were added to a stirred solution of the ester in toluene $(0.3 \mathrm{ml})$. After $18 \mathrm{hrs}$, the solvent was evaporated under reduced pressure to give dimethyl $\left(R, R, R, S, R, 2 R, 2^{\prime} R\right)$-26,26'disulfanediylbis(2-((R)-1-acetoxy-18-((1R,2S)-2-((17S,18S)-17-methoxy-18-methylhexatriacontyl)cyclo-propyl)octadecyl)hexacosa-noate), which showed an identical spectrum to that above. DL-Dithiothreitol $(100 \mathrm{mg})$ was added to a stirred solution of the ester in chloroform $(1 \mathrm{ml})$ followed by the addition of one drop of triethylamine under nitrogen atmosphere. The flask was covered with aluminium foil. The mixture was stirred for $48 \mathrm{hrs}$ at room temperature. The solvent was evaporated. Column chromatography eluting with petrol/ethyl acetate (10:1) to give methyl $(R)-2-((R)-1-$ acetoxy-18-((1R,2S)-2-((17S,18S)-17-methoxy-18-methylhexatriacontyl)cyclopropyl)octadecyl)-26-mercaptohexacosanoate (31) \{MALDI Found [M+Na] $]^{+}:$1364.8; $\mathrm{C}_{88} \mathrm{H}_{172} \mathrm{O}_{5} \mathrm{SNa}$ requires 1364.3 , which showed essentially identical nmr spectra to those of (29) presented above. 
Methyl $(R)-2-((R)-1-($ tert-butyldimethylsilyloxy)-19-((1S,2R)-2-((2S,19S,20S)-19methoxy-20-methyloctatriacontan-2-yl)cyclopropyl)nonadecyl)-26-hydroxyhexacosanoate (34)

(i) Lithium bis-(trimethylsilyl)amide $(0.96 \mathrm{ml}, 1.02 \mathrm{mmol}, 1.06 \mathrm{M})$ was added to a stirred solution of 9-((1S,2R)-2-((2S,19S,20S)-19-methoxy-20-methyloctatriacontan2-yl)cyclopropyl)nonanal (32) [Al Dulayymi, 2013] (0.494 g, $0.651 \mathrm{mmol})$ and methyl (R)-2-((R)-1-(tert-butyldimethylsilyloxy)-10-(1-phenyl-1-tetrazol-5ylsulfonyl)decyl)-26-(pivaloyloxy)hexacosanoate (33) (see supplementary information) $(0.773 \mathrm{~g}, 0.781 \mathrm{mmol})$ in dry THF $(15 \mathrm{ml})$ at $0-5{ }^{\circ} \mathrm{C}$. The solution turned bright yellow/orange and was left to reach room temperature and stirred for $1 \mathrm{hr}$ under $\mathrm{N}_{2}(\mathrm{~g})$ then quenched with sat.aq. $\mathrm{NH}_{4} \mathrm{Cl}(10 \mathrm{ml})$ at $-20{ }^{\circ} \mathrm{C}$. The mixture was extracted with petrol/ethyl acetate $(1: 1,3 \times 15 \mathrm{ml})$ and the combined organic layers were dried and evaporated. Column chromatography (petrol/ethyl acetate, 20:1) gave methyl $(R)$ 2-((R)-1-(tert-butyldimethylsilyloxy)-19-((1S,2R)-2-((2S,19S,20S)-19-methoxy-20methyloctatriacontan-2-yl)cyclopropyl)-nonadec-10-enyl)-26-(pivaloyloxy)hexacosanoate $(0.849 \mathrm{~g} 86 \%)$ as a colourless oil, $[\alpha]_{D}^{23}-8.54\left(c 1.19, \mathrm{CHCl}_{3}\right)$.

(ii) Dipotassium azodicarboxylate (2.5 g) was added in excess to a stirred solution of above olefins $(0.840 \mathrm{~g}, 0.552 \mathrm{mmol})$ in dry THF $(10 \mathrm{ml})$ and methanol $(5 \mathrm{ml})$ at $0{ }^{\circ} \mathrm{C}$ under $\mathrm{N}_{2}(\mathrm{~g})$. Acetic acid $(2 \mathrm{ml})$ in dry THF $(4 \mathrm{ml})$ was added in small portions throughout the day at $0{ }^{\circ} \mathrm{C}$. Further dipotassium azodicarboxylate followed by more of the solution of acetic acid in THF was added. Again, after stirring overnight, more dipotassium azodicarboxylate was added, followed by more acetic acid in THF. After stirring for a further $24 \mathrm{~h}$ the reaction was quenched by adding it in small portions to sat.aq. $\mathrm{NaHCO}_{3}(15 \mathrm{ml})$. The mixture was extracted with petrol/ethyl acetate $(5: 2,3 \mathrm{x}$ $25 \mathrm{ml}$ ) and the combined organic layers were dried and evaporated. Column chromatography (petrol/ethyl acetate, 20:1) gave methyl $(R)-2-((R)-1-($ tert-butyldimethylsilyloxy)-19-((1S,2R)-2-((2S,19S,20S)-19-methoxy-20-methyloctatriacontan2-yl)cyclopropyl)-nonadecyl)-26-(pivaloyloxy)-hexacosanoate $(0.727 \mathrm{~g}, 86 \%)$ as a colourless oil, $[\alpha]_{D}^{21}-6.52\left(c 0.87, \mathrm{CHCl}_{3}\right)$. This showed $\delta_{\mathrm{H}}: 4.05(2 \mathrm{H}, \mathrm{t}, J 6.6 \mathrm{~Hz})$, $3.91(1 \mathrm{H}, \mathrm{dt}, J$ 4.7, $7.0 \mathrm{~Hz}), 3.66(3 \mathrm{H}, \mathrm{s}), 3.35(3 \mathrm{H}, \mathrm{s}), 2.96(1 \mathrm{H}, \mathrm{m}), 2.53(1 \mathrm{H}, \mathrm{ddd}, J$ $3.7,7.2,10.9 \mathrm{~Hz}), 1.62(6 \mathrm{H}, \mathrm{m}), 1.26(147 \mathrm{H}, \mathrm{m}), 0.89(23 \mathrm{H}, \mathrm{m}$, including a singlet at 0.87), $0.67(1 \mathrm{H}, \mathrm{m}), 0.42-0.47(1 \mathrm{H}, \mathrm{m}), 0.10-0.22(3 \mathrm{H}, \mathrm{m}), 0.05(3 \mathrm{H}, \mathrm{s})$ and $0.02(3 \mathrm{H}$, 
s); $\delta_{\mathrm{C}}: 178.7,175.2,143.2,85.5,73.2,64.5,57.7,51.6,51.2,38.7,38.1,37.4,35.3$, 34.5, 32.4, 31.9, 30.5, 30.1, 30.0, 29.9, 29.8, 29.67, 29.63, 29.61, 29.58, 29.54, 29.47, 29.37, 29.2, 29.1, 28.6, 27.6, 27.5, 27.3, 27.2, 26.2, 25.9, 25.7, 22.7, 22.6, 19.7, 18.6, $18.0,14.9,14.1,11.4,10.5,-4.4,-4.9 . v_{\max }: 2924,2853,1733,1464 \mathrm{~cm}^{-1}$.

(iii) The above pivalate $(0.71 \mathrm{~g}, 0.47 \mathrm{mmol})$ was added to a stirred solution of potassium hydroxide $(0.39 \mathrm{~g}, 6.99 \mathrm{mmol})$ in THF $(10 \mathrm{ml})$, methanol $(10 \mathrm{ml})$ and water $(1 \mathrm{ml})$. The mixture was heated under reflux at $70{ }^{\circ} \mathrm{C}$. After $\sim 3$ hours, the reaction was quenched with water $(10 \mathrm{ml})$ and extracted with ethyl acetate $(3 \times 15 \mathrm{ml})$. The combined organic extracts were dried and evaporated. Column chromatography (petrol/ethyl acetate, 10:1) gave methyl $(R)$-2-((R)-1-(tert-butyldimethylsilyloxy)-19$((1 S, 2 R)-2-((2 S, 19 S, 20 S)-19$-methoxy-20-methyloctatriacontan-2-yl)cyclopropyl)nonadecyl)-26-hydroxyhexacosanoate (34) (0.583 g, $86 \%)$ as a white solid, m.p. 27$28{ }^{\circ} \mathrm{C},[\alpha]_{D}^{21}-4.54\left(c \quad 0.69, \mathrm{CHCl}_{3}\right) \quad\left\{\right.$ MALDI Found $(\mathrm{M}+\mathrm{Na})^{+}:$1462.45, $\mathrm{C}_{95} \mathrm{H}_{190} \mathrm{O}_{5} \mathrm{SiNa}$ requires: 1462.43 \}. This showed $\delta_{\mathrm{H}}: 3.91(1 \mathrm{H}, \mathrm{dt}, J 4.8,6.6 \mathrm{~Hz}), 3.66$ $(3 \mathrm{H}, \mathrm{s}), 3.65(2 \mathrm{H}, \mathrm{t}, J 6.6 \mathrm{~Hz}), 3.35(3 \mathrm{H}, \mathrm{s}), 2.96(1 \mathrm{H}, \mathrm{m}), 2.53(1 \mathrm{H}, \mathrm{ddd}, J$ 3.6, 7.2, $10.9 \mathrm{~Hz}), 1.26(141 \mathrm{H}, \mathrm{m}), 1.58$ (9H, br m), $0.91(3 \mathrm{H}, \mathrm{d}, J 6.6 \mathrm{~Hz}), 0.89$ (3H, t, $J 7.1$ $\mathrm{Hz}), 0.87$ (9H, s), $0.86(3 \mathrm{H}, \mathrm{d}, J 7.0 \mathrm{~Hz}), 0.66$ (1H, m), 0.41-0.48 (1H, m), 0.10-0.21 $(3 \mathrm{H}, \mathrm{m}), 0.05(3 \mathrm{H}, \mathrm{s})$ and $0.02(3 \mathrm{H}, \mathrm{s}) ; \delta_{\mathrm{C}}: 176.3,175.2,143.2,85.5,73.3,72.4,63.1$, 57.7, 51.6, 51.2, 38.1, 37.4, 35.3, 34.5, 33.7, 32.8, 32.4, 31.9, 30.5, 30.1, 30.0, 29.9, $29.8,29.72,29.64,29.61,29.52,29.45,29.42,29.38,29.1,28.3,27.8,27.6,27.5$, $27.3,26.2,26.1,25.8,25.5,23.7,22.7,19.7,18.6,18.0,14.9,14.1,10.5,-4.4,-4.9 ; v$ $\max : 3424,2923,2853,1741,1719,1463 \mathrm{~cm}^{-1}$.

Methyl (R)-26-(acetylthio)-2-((R)-1-(tert-butyldimethylsilyloxy)-19-((1S,2R)-2((2S,19S,20S)-19-methoxy-20-methyloctatriacontan-2-yl)cyclopropyl)nonadecyl)

\section{hexacosanoate (35)}

(i) Alcohol (34) $(0.474 \mathrm{~g}, 0.327 \mathrm{mmol})$ and triethylamine $(2 \mathrm{ml})$ in dry dichloromethane $(25 \mathrm{ml})$ was cooled to $-20{ }^{\circ} \mathrm{C}$ under $\mathrm{N}_{2}(\mathrm{~g})$ and stirred for 30 minutes, followed by the addition of $p$-toluenesulfonyl chloride $(0.081 \mathrm{~g}, 0.425 \mathrm{mmol})$ in one portion. The solution was kept in a refrigerator overnight, then the solvent was evaporated. Column chromatography (petrol/ethyl acetate, 10:1) gave methyl $(R)$-2((R)-1-(tert-butyldimethylsilyl)oxy)-19-((1S,2R)-2-((2S,19S,20S)-19-methoxy-20methyloctatriacontan-2-yl)cyclopropyl)-nonadecyl)-26-(tosyloxy)hexacosanoate 
$(0.333 \mathrm{~g}, 65 \%)$ as a colourless oil, $[\alpha]_{D}^{23}-5.03\left(c 0.63, \mathrm{CHCl}_{3}\right)\left\{\right.$ Found $(\mathrm{M}+\mathrm{Na})^{+}$: 1618.15, $\mathrm{C}_{102} \mathrm{H}_{196} \mathrm{O}_{7} \mathrm{SSiNa}$ requires: 1616.44 . This showed $\delta_{\mathrm{H}}: 7.80(2 \mathrm{H}, \mathrm{d}, J 8.2$ $\mathrm{Hz}), 7.36(2 \mathrm{H}, \mathrm{d}, J 7.9 \mathrm{~Hz}), 4.03(2 \mathrm{H}, \mathrm{t}, J 6.5 \mathrm{~Hz}), 3.91(1 \mathrm{H}, \mathrm{dt}, J$ 4.8, $6.6 \mathrm{~Hz}), 3.66$ $\left(3 \mathrm{H}, \mathrm{s}, \mathrm{OCH}_{3}\right), 3.35\left(3 \mathrm{H}, \mathrm{s}, \mathrm{OCH}_{3}\right), 2.96(1 \mathrm{H}, \mathrm{m}), 2.53(1 \mathrm{H}, \mathrm{ddd}, J 3.6,7.2,10.9 \mathrm{~Hz})$, $2.46(3 \mathrm{H}, \mathrm{s}), 1.63(6 \mathrm{H}, \mathrm{m}), 1.26(143 \mathrm{H}, \mathrm{m}), 0.91(3 \mathrm{H}, \mathrm{d}, J 6.6 \mathrm{~Hz}), 0.89(3 \mathrm{H}, \mathrm{t}, J 7.2$ Hz), 0.87 (9H, s), 0.86 (3H, d, J $6.7 \mathrm{~Hz}), 0.66(1 \mathrm{H}, \mathrm{m}), 0.42-0.47(1 \mathrm{H}, \mathrm{m}), 0.09-0.21$ $(3 \mathrm{H}, \mathrm{m}), 0.05(3 \mathrm{H}, \mathrm{s})$ and $0.02(3 \mathrm{H}, \mathrm{s}) ; \delta_{\mathrm{C}}: 175.1,144.5,133.3,129.8,127.9,85.5$, 73.2, 70.7, 60.4, 57.7, 51.6, 51.2, 38.1, 37.4, 35.5, 34.5, 33.7, 32.4, 31.9, 30.5, 30.1, $30.01,30.00,29.9,29.72,29.68,29.62,29.60,29.52,29.47,29.40,29.3,28.9,28.8$, 27.8, 27.6, 27.5, 27.3, 26.2, 26.1, 25.8, 25.3, 23.7, 22.7, 21.6, 19.7, 18.6, 18.0, 14.9, 14.1, 10.5, -4.4, -4.9. $v_{\max }: 2923,2853,1740,1719,1464 \mathrm{~cm}^{-1}$.

(ii) A solution of tosylate $(0.399 \mathrm{~g}, 0.251 \mathrm{mmol})$ and potassium thioacetate $(0.115 \mathrm{~g}$, $1.003 \mathrm{mmol})$ in acetone $(15 \mathrm{ml})$ was stirred at room temperature overnight, then the solvent was evaporated. Column chromatography (petrol/ethyl acetate, 20:1) gave methyl $(R)-26-($ acetylthio)-2-((R)-1-(tert-butyldimethylsilyloxy)-19-((1S,2R)-2-((2S, 19S,20S)-19-methoxy-20-methyloctatriacontan-2-yl)cyclopropyl)nonadecyl)hexacosanoate (35) $(0.227 \mathrm{~g}, 61 \%)$ as a colourless oil, $[\alpha]_{D}^{24}-4.43\left(c 0.73, \mathrm{CHCl}_{3}\right)$ $\left\{\right.$ Found $(\mathrm{M}+\mathrm{Na})^{+}:$1520.42, $\mathrm{C}_{97} \mathrm{H}_{192} \mathrm{O}_{5} \mathrm{SSiNa}$ requires: 1520.42$\}$. This showed $\delta_{\mathrm{H}}$ : $3.90(1 \mathrm{H}, \mathrm{dt}, J 4.7,7.1 \mathrm{~Hz}), 3.65(3 \mathrm{H}, \mathrm{s}), 3.33(3 \mathrm{H}, \mathrm{s}), 2.96(1 \mathrm{H}, \mathrm{m}), 2.85(2 \mathrm{H}, \mathrm{t}, J 7.4$ Hz), $2.52(1 \mathrm{H}, \mathrm{ddd}, J 3.6,7.3,10.9 \mathrm{~Hz}), 2.31(3 \mathrm{H}, \mathrm{s}), 1.55(6 \mathrm{H}, \mathrm{m}), 1.26(143 \mathrm{H}, \mathrm{m})$, $0.90(3 \mathrm{H}, \mathrm{d}, J 6.9 \mathrm{~Hz}), 0.88(3 \mathrm{H}, \mathrm{t}, J 6.6 \mathrm{~Hz}), 0.86(9 \mathrm{H}, \mathrm{s}), 0.85(3 \mathrm{H}, \mathrm{d}, J 7.0 \mathrm{~Hz}), 0.65$ $(1 \mathrm{H}, \mathrm{m}), 0.41-0.48(1 \mathrm{H}, \mathrm{m}), 0.09-0.20(3 \mathrm{H}, \mathrm{m}), 0.04(3 \mathrm{H}, \mathrm{s})$ and $0.02(3 \mathrm{H}, \mathrm{s}) ; \delta_{\mathrm{C}}$ : 195.9, 175.1, 85.4, 73.4, 73.2, 71.0, 57.7, 51.6, 51.2, 38.1, 37.7, 37.4, 35.3, 34.5, 33.7, 32.8, 32.8, 32.6, 31.9, 30.6, 30.5, 30.4, 30.1, 30.00, 29.96, 29.84, 29.73, 29.67, 29.64, $29.62,29.60,29.57,29.53,29.50,29.46,29.39,29.1,28.8,27.8,27.6,27.5,27.3$, 26.2, 26.1, 25.7, 23.7, 22.7,19.7, 18.6, 17.9, 14.9, 14.1, 10.5, -4.3, -4.9. $v_{\max }: 2921$, $2851,1731,1643,1463 \mathrm{~cm}^{-1}$.

\section{Methyl (R)-26-(acetylthio)-2-((R)-1-hydroxy-19-((1S,2R)-2-((2S,19S,20S)-19-}

\section{methoxy-20-methyloctatriacontan-2-yl)cyclopropyl)nonadecyl)hexacosanoate}

Thioacetate (35) (50 mg, $0.0333 \mathrm{mmol})$ was dissolved in dry THF (4 ml) in a dry polyethylene vial under $\mathrm{N}_{2}(\mathrm{~g})$ at $0{ }^{\circ} \mathrm{C}$. Pyridine $(98.2 \mathrm{mg}, 7.77 \mathrm{mmol}, 0.1 \mathrm{ml})$ and HF.Pyridine $(88 \mathrm{mg}, 0.8 \mathrm{ml})$ were added and the mixture was stirred at $45{ }^{\circ} \mathrm{C}$ 
overnight then added slowly to sat.aq. $\mathrm{NaHCO}_{3}(10 \mathrm{ml})$. The solution was extracted with petrol/ethyl acetate $(1: 1,3 \times 15 \mathrm{ml})$ and the combined organic extracts were dried, filtered and evaporated. Column chromatography (petrol/ethyl acetate, 10:1) gave methyl $(R)-26-($ acetylthio)-2- $((R)-1$-hydroxy-19-((1S,2R)-2-((2S,19S,20S)-19methoxy-20-methyloctatriacontan-2-yl)cyclopropyl)nonadecyl)-hexacosanoate (41.1 mg, $90 \%)$ as a white solid, m.p. $41-43{ }^{\circ} \mathrm{C},[\alpha]_{D}^{21}-2.27\left(c\right.$ 2.14, $\left.\mathrm{CHCl}_{3}\right)$ \{Found $(\mathrm{M}+$ $\mathrm{Na})^{+}:$1406.33, $\mathrm{C}_{91} \mathrm{H}_{178} \mathrm{O}_{5} \mathrm{SNa}$ requires: 1406.33$\}$. This showed $\delta_{\mathrm{H}}: 3.72(3 \mathrm{H}, \mathrm{s}), 3.66$ $(1 \mathrm{H}, \mathrm{m}), 3.35\left(3 \mathrm{H}, \mathrm{s}, \mathrm{OCH}_{3}\right), 2.96(1 \mathrm{H}, \mathrm{m}), 2.87(2 \mathrm{H}, \mathrm{t}, J$ 7.4), $2.45(1 \mathrm{H}, \mathrm{dt}, J$ 5.4, 9.1), 2.33 (3H, s), 1.58 (8H, m), $1.26(142 \mathrm{H}, \mathrm{m}), 0.91(3 \mathrm{H}, \mathrm{d}, J 6.6 \mathrm{~Hz}), 0.89$ (3H, t, $J$ $6.9 \mathrm{~Hz}), 0.86(3 \mathrm{H}, \mathrm{d}, J 6.9 \mathrm{~Hz}), 0.66(1 \mathrm{H}, \mathrm{m}), 0.41-0.48(1 \mathrm{H}, \mathrm{m})$ and $0.09-0.21(3 \mathrm{H}$, m); $\delta_{\mathrm{C}}: 196.1,176.3,85.5,76.6,72.3,57.7,51.5,50.9,38.1,37.4,35.7,35.3,34.5$, $32.4,31.9,30.6,30.5,30.09$, 30.05, 30.00, 29.96, 29.72, 29.66, 29.62, 29.59, 29.57, $29.51,29.44,29.38,29.2,29.1,28.8,27.6,27.4,27.3,26.2,26.1,25.7,22.7,19.7$, $18.6,14.9,14.1 ; v_{\max }: 3418,2922,2851,1709,1687,1465 \mathrm{~cm}^{-1}$.

\section{$\left(S, S, S, R, S, R, 2 R, 2 R^{\prime}\right)-26-26$ '-Disulfanediylbis(2-((R)-1-hydroxy-19-((1S,2R)-2-} $((2 S, 19 S, 20 S)-19-m e t h o x y-20$-methyloctatriacontan-2-yl)cyclopropyl) nona-

\section{decyl)hexacosanoic acid (36)}

Methyl ～(R)-26-(acetylthio)-2-((R)-1-hydroxy-19-((1S,2R)-2-((2S,19S,20S)-19methoxy-20-methyloctatriacontan-2-yl)cyclopropyl)nonadecyl)hexacosanoate (14 $\mathrm{mg}, 0.010 \mathrm{mmol})$ was suspended in $5 \%$ aq. TBAH $(2 \mathrm{ml})$ and heated to $100{ }^{\circ} \mathrm{C}$ overnight. The solution was cooled to room temperature, acidified to $\mathrm{pH} 1$ with $1 \mathrm{M}$ $\mathrm{HCl}$ and extracted with diethyl ether $(3 \times 15 \mathrm{ml})$. The combined organic layers were dried, filtered and the solvent evaporated. Column chromatography (chloroform/ methanol, 10:1) gave ( $\left.S, S, S, R, S, R, 2 R, 2 R^{\prime}\right)$-26-26'-disulfanediylbis(2-((R)-1-hydroxy19-((1S,2R)-2-((2S,19S,20S)-19-methoxy-20-methyloctatriacontan-2-yl)cyclopropyl)nonadecyl)hexacosanoic acid (36) $(7.7 \mathrm{mg}, 58 \%)$ as a white solid, $[\alpha]_{D}^{22}-2.78(c$ 0.77, $\left.\mathrm{CHCl}_{3}\right)$. This showed $\delta_{\mathrm{H}}: 3.91(1 \mathrm{H}, \mathrm{m}), 3.35(3 \mathrm{H}, \mathrm{s}), 2.97(1 \mathrm{H}, \mathrm{m}), 2.69(2 \mathrm{H}, \mathrm{t}, J$ $7.4 \mathrm{~Hz}), 2.47(1 \mathrm{H}, \mathrm{dt}, J$ 5.4, $9.1 \mathrm{~Hz}), 1.67(8 \mathrm{H}, \mathrm{m}), 1.26(144 \mathrm{H}, \mathrm{m}), 0.90(3 \mathrm{H}, \mathrm{d}, J 6.6$ $H z), 0.89(3 \mathrm{H}, \mathrm{t}, J 7.0 \mathrm{~Hz}), 0.86(3 \mathrm{H}, \mathrm{d}, J 6.9 \mathrm{~Hz}), 0.66(1 \mathrm{H}, \mathrm{m}), 0.44(1 \mathrm{H}, \mathrm{m})$ and 0.08-0.20 (3H, m); $\delta_{\mathrm{C}}: 85.5,57.9,50.9,45.3,39.4,38.1,37.4,35.3,34.5,32.4,31.9$, 30.9, 30.5, 30.1, 30.0, 29.9, 29.5, 29.4, 29.2, 28.5, 27.6, 27.3, 26.1, 22.7, 19.77, 18.6, $14.9,14.1,10.5,8.6 ; v_{\max }: 3423,2924,2852,1718,1465 \mathrm{~cm}^{-1}$. 
Methyl $(R)-$ 2-((R)-1-(tert-butyldimethylsilyloxy)-12-((1R,2S)-2-(14-((1R,2S)-2eicosylcyclopropyl)-tetradecyl)-cyclopropyl)dodecyl)-26-(pivaloyloxy) hexacosanoate (38)

(i) Lithium bis(trimethylsilyl)amide $(0.734 \mathrm{ml}, 0.775 \mathrm{mmol})$ was added to a stirred solution of aldehyde (19) $(0.35 \mathrm{~g}, 0.43 \mathrm{mmol})$ and sulfone (37) (0.405 g, 0.515 $\mathrm{mmol})$ in dry THF $(30 \mathrm{ml})$ at $-10{ }^{\circ} \mathrm{C}$ under $\mathrm{N}_{2}$. The reaction turned bright yellow and was left to reach room temperature and stirred for $1 \mathrm{~h}$ under $\mathrm{N}_{2}$. The reaction was quenched by adding sat.aq. $\mathrm{NH}_{4} \mathrm{Cl}$. The product was extracted with petroleum/ethyl acetate $(10: 1,3 \times 100 \mathrm{ml})$ dried and evaporated. Column chromatography eluting with petrol/ethyl acetate $(20: 1)$ gave a semi-solid, methyl $(E / Z)-(R)-2-((R)-1-($ tertbutyldimethylsilyloxy)-12-((1S,2S)-2-(14-((1R,2S)-2-eicosyl-cyclopropyl)tetradecyl)cyclopropyl)dodec-10-enyl)-26-(pivaloyloxy)hexa-cosanoate as a mixture of two isomers in ratio $(3: 1)(0.35 \mathrm{~g}, 60 \%)$.

(ii) Dipotassium azodicarboxylate $(2 \mathrm{~g}, 10.3 \mathrm{mmol})$ was added to a stirred solution of the alkene above $(0.34 \mathrm{~g}, 0.249 \mathrm{mmol})$ in THF $(20 \mathrm{ml})$ and methanol $(4 \mathrm{ml})$ at $5{ }^{\circ} \mathrm{C}$. A solution of glacial acetic acid $(2.5 \mathrm{ml})$ and THF $(2.5 \mathrm{ml})$ was prepared and half was added at $5{ }^{\circ} \mathrm{C}$ dropwise and the mixture was stirred at r.t. for $2 \mathrm{hrs}$. The other half was added at r.t. and the mixture was stirred overnight. Dipotassium azodicarboxylate (2.0 g) and glacial acetic acid $(2 \mathrm{ml})$ were added and stirred overnight. This mixture was slowly added to sat.aq. $\mathrm{NH}_{4} \mathrm{Cl}$ and extracted with petroleum/ethyl acetate $(1: 1,3 \mathrm{x}$ $100 \mathrm{ml})$ and the combined organic layers were washed with water $(100 \mathrm{ml})$ and the solvent was evaporated. The procedure was repeated. Column chromatography eluting with petroleum/ethyl acetate (5:1) gave methyl $(R)-2-((R)-1$-(tertbutyldimethylsilyloxy)-12-((1R,2S)-2-(14-((1R,2S)-2-eicosylcyclopropyl)tetradecyl)cyclopropyl)dodecyl)-26-(pivaloyloxy)hexacosanoate (38) as a white solid (0.310 g, $91 \%), \mathrm{mp} 40-42{ }^{0} \mathrm{C},[\alpha]^{21}{ }_{\mathrm{D}}=+1.44\left(c \quad 0.9, \mathrm{CHCl}_{3}\right)$ \{Found $(\mathrm{M}+\mathrm{Na})^{+}: 1388.3154$ $\mathrm{C}_{90} \mathrm{H}_{176} \mathrm{O}_{5} \mathrm{SiNa}$ requires 1388.3179$\}$ which showed $\delta_{\mathrm{H}}\left(500 \mathrm{MHz}, \mathrm{CDCl}_{3}\right): 4.05(2 \mathrm{H}, \mathrm{t}$, $J 6.6 \mathrm{~Hz}), 3.92-3.89(1 \mathrm{H}, \mathrm{m}), 3.66(3 \mathrm{H}, \mathrm{s}), 2.54-2.50(1 \mathrm{H}, \mathrm{m}), 1.37(139 \mathrm{H}, \mathrm{m}$, including $\mathrm{s}$ at 1.20), 0.90-0.82 (16H, m, including s at 0.86), 0.68-0.61 $(4 \mathrm{H}, \mathrm{m}), 0.56$ $(2 \mathrm{H}, \mathrm{dt}, J 4.1,8.2 \mathrm{~Hz}), 0.05(3 \mathrm{H}, \mathrm{s}), 0.02(3 \mathrm{H}, \mathrm{s}),-0.32(2 \mathrm{H}$, br.q, $J 5.0 \mathrm{~Hz}) ; \delta_{\mathrm{C}}$ 178.6, 175.1, 64.4, 51.6, 51.2, 38.7, 33.7, 31.9, 30.2, 29.8, 29.7, 29.6, 29.5, 29.4, 29.3, 29.2, 28.7, 28.6, 27.8, 27.5, 27.2, 25.7, 23.7, 22.6, 22.3, 17.9, 15.7, 14.0, 10.9; $v_{\text {max }}$ $2920,2852,1732,1638,1464,1363,1284,1253,1161,836,775,720 \mathrm{~cm}^{-1}$. 
Methyl $(R)-2-((R)-1-(t e r t-b u t y l d i m e t h y l s i l y l o x y)-12-((1 R, 2 S)-2-(14-((1 R, 2 S)-2-$ eicosylcyclopropyl)tetradecyl)cyclopropyl)dodecyl)-26-(hydroxy)hexacosanoate (39)

(ii) The above ester $(0.310 \mathrm{~g}, 0.224 \mathrm{mmol})$ in $(3 \mathrm{ml})$ THF was added to a stirred solution of potassium hydroxide $(0.19 \mathrm{~g}, 3.57 \mathrm{mmol})$ in a mixture of THF $(10 \mathrm{ml})$, methanol $(10 \mathrm{ml})$ and water $(1 \mathrm{ml})$, then heated under reflux at 70 or $80{ }^{\circ} \mathrm{C}$. After 3 hrs, the reaction was quenched with water $(10 \mathrm{ml})$ and extracted with petroleum/ethyl acetate $(10: 1,3 \times 25 \mathrm{ml})$. The combined organic extracts were dried, filtered and evaporated. Column chromatography with petroleum/ethyl acetate (10:1) gave methyl $(R)-2-((R)-1-($ tert-butyldimethylsilyloxy)-12-((1R,2S)-2-(14-((1R,2S)-2-eicosylcyclopropyl)tetradecyl)cyclopropyl)dodecyl)-26-hydroxyhexacosanoate (39) as a white solid $(0.196 \mathrm{~g}, 67 \%), \mathrm{mp} 42-43{ }^{0} \mathrm{C},[\alpha]_{\mathrm{D}}^{22}=+1.23\left(c\right.$ 1.1, $\left.\mathrm{CHCl}_{3}\right)\left\{\right.$ Found $(\mathrm{M}+\mathrm{Na})^{+}$: 1304.2560. $\mathrm{C}_{85} \mathrm{H}_{168} \mathrm{O}_{4} \mathrm{SiNa}$ requires 1304.2604$\}$ which showed $\delta_{\mathrm{H}}\left(500 \mathrm{MHz}, \mathrm{CDCl}_{3}\right)$ : 3.92-3.89 (1H, m), $3.66(3 \mathrm{H}, \mathrm{s}), 3.65(2 \mathrm{H}, \mathrm{t}, J 6.6 \mathrm{~Hz}), 2.55-2.51(1 \mathrm{H}, \mathrm{m}), 1.60-1.14$ (125H, br.m), 0.91-0.82 (22H, m, including s at 0.89), 0.66-0.61 $(4 \mathrm{H}, \mathrm{m}), 0.57(2 \mathrm{H}$, dt, J 3.75, $7.85 \mathrm{~Hz}), 0.05(3 \mathrm{H}, \mathrm{s}), 0.02(3 \mathrm{H}, \mathrm{s}),-0.32(2 \mathrm{H}$, br.q, $J 5.05 \mathrm{~Hz}) ; \delta_{\mathrm{C}}: 175.1$, 63.0, 51.5, 51.1, 34.1, 33.7, 32.8, 31.9, 30.8, 30.2, 29.8, 29.7, 29.6, 29.5, 29.4, 28.7, 27.8, 27.4, 25.7, 23.7, 22.6, 17.9, 15.7, 14.9, 10.9, - 4.3,- 4.9; $v_{\max } / 3414,2918,2850$, $1739,1638,1469,1384,1167,836,720,617 \mathrm{~cm}^{-1}$.

Methyl (R)-26-(acetylthio)-2-((R)-1-(tert-butyldimethylsilyloxy)-12-((1R,2S)-2(14-((1R,2S)-2-eicosylcyclopropyl)tetradecyl)cyclopropyl)dodecyl) hexacosanoate (40)

(i) Methyl $(R)$ - 2-((R)-1-(tert-butyldimethylsilyloxy)-12-((1R,2S)-2-(14-((1R,2S)-2eicosylcyclopropyl)tetradecyl)cyclopropyl)dodecyl)-26-hydroxyhexacosanoate (0.196 $\mathrm{g}, 0.53 \mathrm{mmol})$ and triethylamine $(1.5 \mathrm{ml})$ in dry dichloromethane $(10 \mathrm{ml})$ was cooled to $-20{ }^{0} \mathrm{C}$ under $\mathrm{N}_{2}$, and stirred for $30 \mathrm{~min}$, followed by the addition of toluenesulfonyl chloride $(0.034 \mathrm{~g}, 0.175 \mathrm{mmol})$ in one portion. The solution was kept in a refrigerator overnight then the solvent was evaporated. Column chromatography (petroleum/ethyl acetate, 10:1) gave methyl $(R)$-2-((R)-1-(tert-butyldimethylsilyloxy)12-((1R,2S)-2-(14-((1R,2S)-2-eicosylcyclopropyl)tetradecyl)-cyclopropyl)dodecyl)26-(tosyloxy)hexacosanoate $(0.155 \mathrm{~g}, 71 \%)$ as a thick oil which soldified later, $[\alpha]^{22}$ $=+1.10\left(c 0.103, \mathrm{CHCl}_{3}\right)\left\{\right.$ Found $(\mathrm{M}+\mathrm{Na})^{+}:$1458.2668; $\mathrm{C}_{92} \mathrm{H}_{174} \mathrm{O}_{6} \mathrm{SSiNa}$ requires: 
1458.2693 \}, which showed $\delta_{\mathrm{H}}$ : (500,MHz, $\left.\mathrm{CDCl}_{3}\right): 7.70(2 \mathrm{H}, \mathrm{d}, J 8.2 \mathrm{~Hz}), 7.34(2 \mathrm{H}$, d, J 7.85 Hz), $4.02(2 \mathrm{H}, \mathrm{t}, J 6.6 \mathrm{~Hz}), 3.92-3.87(1 \mathrm{H}, \mathrm{m}), 3.65(3 \mathrm{H}, \mathrm{s}), 2.55-2.51(1 \mathrm{H}$, m), $2.45(3 \mathrm{H}, \mathrm{s}), 1.63(2 \mathrm{H}$, pent, $J 6.6 \mathrm{~Hz}), 1.38-1.20(129 \mathrm{H}, \mathrm{m}), 0.90-0.82(15 \mathrm{H}, \mathrm{m}$, including s at 0.87), 0.66-0.61 $(4 \mathrm{H}, \mathrm{m}), 0.56(2 \mathrm{H}, \mathrm{dt}, J 4.1,8.15 \mathrm{~Hz}), 0.05(3 \mathrm{H}, \mathrm{s})$, $0.02(3 \mathrm{H}, \mathrm{s}), 0.32(2 \mathrm{H}$, br.q, $J 5.05 \mathrm{~Hz})$; $\delta_{\mathrm{C}}: 175.0,144.5,133.3,129.7,127.8,73.2$, 70.6, 51.5, 51.1, 33.6, 31.9, 30.2, 29.8, 29.6, 29.5, 29.4, 29.3, 28.9, 28.8, 28.7, 27.8, $27.4,25.7,25.3,23.7,22.6,21.5,17.9,15.7,14.0,10.9,-4.3,-4.9 ; v_{\max } / 2923,2852$, $1738,1644,1464,1366,1177,719 \mathrm{~cm}^{-1}$.

(ii) The above ester $(0.150 \mathrm{~g}, 0.105 \mathrm{mmol})$ dissolved in $(3 \mathrm{ml})$ dry THF and $(7 \mathrm{ml})$ acetone. Potassium thioacetate $(0.06 \mathrm{~g}, 0.422 \mathrm{mmol})$ was added and the solution was stirred at room temperature overnight then the solvent was evaporated. Column chromatography (petroleum/ethyl acetate, 20:1) gave methyl (R)-26-(acetylthio)-2$((R)-1$-(tert-butyldimethylsilyloxy)-12-((1R,2S)-2-(14-((1R,2S)-2-eicosylcyclopropyl)tetradecyl)-cyclopropyl)dodecyl)hexacosanoate (40) (0.136 g, $94 \%$ ) as a pale yellow thick oil which soldified later, $[\alpha]^{20}{ }_{\mathrm{D}}=+1.07\left(c 0.91 \mathrm{~g}, \mathrm{CHCl}_{3}\right)\{$ Found $(\mathrm{M}+\mathrm{Na})^{+}:$1362.2460, $\mathrm{C}_{87} \mathrm{H}_{170} \mathrm{O}_{4} \mathrm{SSiNa}$ requires: 1362.2481$\}$ which showed $\delta_{\mathrm{H}}(500$,

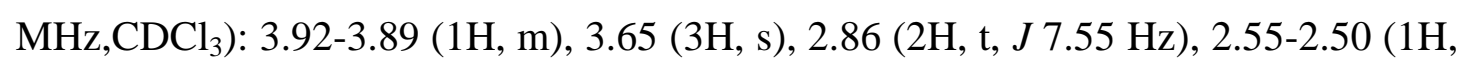
m), $2.31(3 \mathrm{H}, \mathrm{s}), 1.58-1.14(126 \mathrm{H}, \mathrm{m}), 0.89-0.83(20 \mathrm{H}, \mathrm{m}$, including s at 0.86), 0.65$0.61(4 \mathrm{H}, \mathrm{m}), 0.56(2 \mathrm{H}, \mathrm{dt}, J 4.1 \mathrm{~Hz}), 0.04(3 \mathrm{H}, \mathrm{s}), 0.02(3 \mathrm{H}, \mathrm{s}),-0.32$ (2H, br.q, $J$ $4.75 \mathrm{~Hz})$; $\delta_{\mathrm{C}}: 195.7,175.0,73.2,51.5,51.1,41.3,36.0,33.6,31.9,30.5,30.2,29.8$, 29.7, 29.6, 29.5, 29.4, 29.3, 29.1, 29.0, 28.8, 28.7, 27.8, 27.6, 27.5, 25.8, 25.7, 23.6, $22.7,22.5,20.4,19.4,18.7,17.9,14.2,11.4,10.9,-4.3,-4.9 ; v /_{\max }: 2918,2849,1738$, $1694,1465,1360,1253,836,720 \mathrm{~cm}^{-1}$.

\section{Methyl $(R)-26-($ acetylthio)-2-((R)-1-hydroxy-12-((1R,2S)-2-(14- $((1 R, 2 S)-2-$}

\section{eicosylcyclopropyl)tetradecyl)cyclopropyl)dodecyl)hexacosanoate}

Methyl $\quad(R)$-26-(acetylthio)-2-((R)-1-(tert-butyldimethylsilyloxy)-12-((1R,2S)-2-(14$((1 R, 2 S)$-2-eicosylcyclopropyl)tetradecyl)cyclopropyl)dodecyl)hexacosanoate $\quad(0.130$ $\mathrm{g}, 0.098 \mathrm{mmol})$ was dissolved in dry THF $(10 \mathrm{ml})$ in a dry polyethylene vial under $\mathrm{N}_{2}$ at $0{ }^{0} \mathrm{C}$. Pyridine $(98.2 \mathrm{mg}, 7.77 \mathrm{mmol}, 0.1 \mathrm{ml})$ and hydrogen fluoride-pyridine complex $(88 \mathrm{mg}, 0.8 \mathrm{ml})$ were added and the mixture stirred at $45{ }^{0} \mathrm{C}$ overnight, then, the mixture was added slowly to a sat. aq. $\mathrm{NaHCO}_{3}(15 \mathrm{ml})$. The solution was extracted with petroleum/ ethylacetate $(5: 1,3 \times 50 \mathrm{ml})$ and the combined organic 
extracts were dried, filtered and evaporated. Column chromatography (petroleum/ ethyl acetate, 10:1) gave methyl $(R)$ - 26-(acetylthio)-2-(( $R)-1$-hydroxy-12-((1R,2S)-2(14-((1R,2S)-2-eicosylcyclopropyl)tetradecyl)cyclopropyl)dodecyl)hexacosanoate $(0.065 \mathrm{~g}, 55 \%)$ as a thick oil which soldified later, $[\alpha]^{20}{ }_{\mathrm{D}}=+1.59\left(c 1.1, \mathrm{CHCl}_{3}\right)$, \{Found $(\mathrm{M}+\mathrm{Na})^{+}: 1248.1271 \mathrm{C}_{81} \mathrm{H}_{156} \mathrm{O}_{4} \mathrm{SNa}$ requires: 1248.1047$)$, which showed $\delta_{\mathrm{H}}($ $\left.500 \mathrm{MHz}, \mathrm{CDCl}_{3}\right) ; 3.71(3 \mathrm{H}, \mathrm{s}), 3.67-3.64(1 \mathrm{H}, \mathrm{m}), 2.86(2 \mathrm{H}, \mathrm{t}, J 7.25 \mathrm{~Hz}), 2.45-2.42$ $(1 \mathrm{H}, \mathrm{m}), 2.32(3 \mathrm{H}, \mathrm{s}), 1.73-1.69(1 \mathrm{H}, \mathrm{m}), 1.60-1.50(2 \mathrm{H}, \mathrm{m}), 1.37-1.14(132 \mathrm{H}, \mathrm{m})$, 0.89-0.83 (3H, m), 0.64-0.60 (4H, m), $0.56(2 \mathrm{H}, \mathrm{dt}, J 4.1,8.2 \mathrm{~Hz}),-0.32$ (2H, br.q, $J$ $5.05 \mathrm{~Hz})$; $\delta_{\mathrm{C}}: 195.9,176.2,72.2,51.4,50.9,41.3,35.6,31.9,30.5,30.2$, 29.7, 29.6, 29.5, 29.4, 29.3, 29.1, 29.0, 28.8, 28.7, 27.4, 25.7, 22.6, 22.5, 20.4, 15.7, 14.2, 14.1, $11.4,10.8 ; v / \max : 2916,2849,1695,1469,1360,1166,836,720 \mathrm{~cm}^{-1}$.

\section{$\left(S, R, S, R, R, 2 R, 2^{\prime} R\right)-26,26 '-D i s u l f a n e d i y l b i s(2-((R)-1-h y d r o x y-12-((1 R, 2 S)-2-(14-$} $((1 R, 2 S)$-2-eicosylcyclopropyl)tetradecyl)cyclopropyl)dodecyl)hexacosanoic acid) (41)

Methyl $(R)-26-($ acetylthio)-2-((R)-1-hydroxy-12-((1R,2S)-2-(14-((1R,2S)-2-eicosylcy clopropyl)tetradecyl)cyclopropyl)dodecyl)hexacosanoate $(0.06 \mathrm{~g}, 0.048 \mathrm{mmol})$ was suspended in $5 \%$ aq. TBAH $(10 \mathrm{ml})$ and the solution was heated to $100{ }^{0} \mathrm{C}$ overnight. The solution was cooled to room temperature and acidified to $\mathrm{pH} 1$ with $1 \mathrm{M} \mathrm{HCl}$ and then extracted with diethyl ether (added petroleum) $(3 \times 30 \mathrm{ml})$. The combined organic layers were filtered and the solvent evaporated. Column chromatography (chloroform/methanol, 10:1) gave $\left(S, R, S, R, R, 2 R, 2^{\prime} R\right)$-26,26'-disulfanediylbis(2-((R)1-hydroxy-12-((1R,2S)-2-(14-((1R,2S)-2-eicosylcyclopropyl)tetradecyl)cyclopropyl) dodecyl)hexacosanoic acid) (41) $(0.02 \mathrm{~g}, 33 \%)$ as a white solid, mp 54-56 ${ }^{0} \mathrm{C},[\alpha]^{22}$ $=+1.64\left(\mathrm{c} 0.50, \mathrm{CHCl}_{3}\right) ; \delta_{\mathrm{H}}:\left(500 \mathrm{MHz}, \mathrm{CDCl}_{3}+\right.$ few drops of $\left.\mathrm{CD}_{3} \mathrm{OD}\right): 3.69-3.64$ $(2 \mathrm{H}, \mathrm{m}), 2.67$ (4H, br.t, J 7.55 Hz), 2.43-2.39 (2H, m), 1.71-1.63 (12H, m), $1.52(24 \mathrm{H}$, br.s), $1.36-1.04(236 \mathrm{H}, \mathrm{m}), 0.87(6 \mathrm{H}, \mathrm{t}, J 6.6 \mathrm{~Hz}), 0.64-0.63(8 \mathrm{H}, \mathrm{m}), 0.55(4 \mathrm{H}, \mathrm{dt}, J$ $4.1,8.2 \mathrm{~Hz}),-0.33(4 \mathrm{H}, \mathrm{q}, J 5.35 \mathrm{~Hz}), \delta_{\mathrm{C}}: 176.2,72.0,39.2,35.5,31.8,30.1,29.6$, $29.5,29.4,29.3,29.1,28.6,28.4,27.3,25.7,22.6,15.7,14.0,10.8 ; v / m_{\max }: 3442,2917$, $2850,1717,1469,1400,1170,720 \mathrm{~cm}^{-1}$. 
Methyl $(R)-2-((R)-12-((1 R, 2 S)-2-(14-((1 R, 2 S)-2-e i c o s y l c y c l o p r o p y l)-t e t r a-$ decyl)cyclopropyl)-1-(prop-1-en-2-yloxy)dodecyl)-26-mercaptohexacosanoate (42)

Excess diazomethane in ether was added to $\left(S, R, S, R, R, 2 R, 2^{\prime} R\right)-26,26$ '-disulfanediylbis(2-((R)-1-hydroxy-12-((1R,2S)-2-(14-((1R,2S)-2-eicosylcyclo-propyl)tetradecyl)cyclopropyl)dodecyl)hexacosanoic acid) and stirred for $30 \mathrm{~min}$. The solvent was evaporated to give dimethyl $\left(S, R, S, R, R, 2 R, 2^{\prime} R\right)-26,26$ '-disulfanediylbis(2-((R)-1hydroxy-12-((1R,2S)-2-(14-((1R,2S)-2-eicosylcyclopropyl)tetradecyl)cyclopropyl)dodecyl)hexacosanoate)). This was used for next step without purification; acetic anhydride $(0.3 \mathrm{ml})$ and pyridine $(0.3 \mathrm{ml})$ were added to a stirred solution of the ester in toluene $(0.3 \mathrm{ml})$. The mixture was stirred for $18 \mathrm{hrs}$ then the solvent was evaporated to give dimethyl $\left(S, R, S, R, R, 2 R, 2^{\prime} R\right)-26,26$ '-disulfanediylbis(2-(( $\left.R\right)-1$ acetoxy-12-((1R,2S)-2-(14-((1R,2S)-2-eicosylcyclopropyl)-tetradecyl)cyclopropyl)dodecyl)hexacosanoate), which showed $\delta_{\mathrm{H}}:\left(400 \mathrm{MHz}, \mathrm{CDCl}_{3}\right): 5.11-5.06(2 \mathrm{H}, \mathrm{m})$, $3.68(6 \mathrm{H}, \mathrm{s}), 2.68(4 \mathrm{H}, \mathrm{t}, J 7.4 \mathrm{~Hz}), 2.64-2.59(2 \mathrm{H}, \mathrm{m}), 2.03(6 \mathrm{H}, \mathrm{s}), 1.69-1.11(276 \mathrm{H}$, m), 0.88 (6H, t, $J 6.28 \mathrm{~Hz}), 0.65$ (4H, m), 0.57 (4H, dt, $J 3.88 \mathrm{~Hz}),-0.32$ (4H, br.q, $J$ $4.88 \mathrm{~Hz}$ ). The product was used for next step without purification. DL-Dithiothreitol $(100 \mathrm{mg})$ was added to a stirred solution of the ester in chloroform $(1 \mathrm{ml})$ followed by the addition of one drop of triethylamine under nitrogen. The flask was covered with aluminium foil. The mixture was stirred for $48 \mathrm{hrs}$ at room temperature, Then the solvent was evaporated. Column chromatography eluting with petrol/ethyl acetate (10:1) gave methyl $(R)-2-((R)-1$-acetoxy-12-((1R,2S)-2-(14- $((1 R, 2 S)-2$-eicosylcyclopropyl)tetradecyl)cyclopropyl)dodecyl)-26-mercaptohexacosanoate (41) \{Found (MALDI) $[\mathrm{M}+\mathrm{Na}]^{+}: 1248.92 ; \mathrm{C}_{81} \mathrm{H}_{156} \mathrm{NaO}_{4} \mathrm{~S}$ requires: 1249.16$\}$, which showed $\delta_{\mathrm{H}}$ : $\left(400 \mathrm{MHz}, \mathrm{CDCl}_{3}\right): 5.11-5.06(1 \mathrm{H}, \mathrm{m}), 3.68(3 \mathrm{H}, \mathrm{s}), 2.64-2.59(1 \mathrm{H}, \mathrm{m}), 2.52(2 \mathrm{H}, \mathrm{q}, J$ $7.4 \mathrm{~Hz}), 2.02(3 \mathrm{H}, \mathrm{s}) 1.63-1.11(137 \mathrm{H}, \mathrm{m}), 0.88(3 \mathrm{H}, \mathrm{t}, J 6.52 \mathrm{~Hz}), 0.68-0.64(2 \mathrm{H}, \mathrm{m})$, $0.57(2 \mathrm{H}, \mathrm{dt}, J 3.88, \mathrm{~Hz}),-0.32(2 \mathrm{H}$, br.q, $J 5.24 \mathrm{~Hz})$.

\section{References:}

Al Dulayymi, J.R., Baird, M.S., Roberts, E., 2003. The synthesis of a single enantiomer of a major $\alpha$-mycolic acid of Mycobacterium tuberculosis. J.Chem. Soc.,Chem.Commun., 228229. 
Al Dulayymi, J.R., Baird, M.S., Roberts, E., 2005. The synthesis of a single enantiomer of a major $\alpha$-mycolic acid of M. tuberculosis. Tetrahedron, 61, 11939-11951.

Al Dulayymi, J.R., Baird, M.S., Roberts, E., Minnikin, D.E., 2006a. Tetrahedron 2006, 62, 11867-11880.

Al-Dulayymi, J.R., Baird, M.S., Mohammed, H., Roberts, E., Clegg, W., 2006b The synthesis of one enantiomer of the $\alpha$-methyl-trans-cyclopropane unit of mycolic acids. Tetrahedron,62, 4851-4862.

Al Dulayymi, J.R., Baird, M.S., Roberts, E., Deysel, M., Verschoor, J., 2007. The first syntheses of single enantiomers of the major methoxymycolic acid of Mycobacterium tuberculosis. Tetrahedron, 63, 2571-2592.

Al Dulayymi, J.R., Baird, M.S., Theunissen,C., Rowles, R.R., submitted for publication.

Angelova, P., Kostova, K., Hinrichs, K., Tsankov, D., 2005. A convenient synthesis of longchain 4-substituted benzyloxycarbonyl alkanethiols for the formation of self assembled monolayers on metal substrates. Cent.Eur.J.Chem., 3, 658-667.

Anklam, E., Aced, A., 1990. NMR and Mass spectroscopic studies on n-(alkylthio)-1alkanethiols. J.Agric.Food Chem., 38, 123-125.

Barry, C. E., Lee, R. E., Mdluli, K., Sampson, A. E., Schroeder, B. G., Slayden, R. A., Yuan, Y., 1998. Mycolic acids: Structure, biosynthesis and physiological functions. Prog. Lipid Res., 37, 143-179.

Benadie, Y. Deysel, M., Siko, D.G.R., Roberts, V.V., Van Wyngaardt, S., Thanyani, S.T., Sekanka, G., Ten Bokum, A.M.C., Collett, L.A., Grooten, J., Baird, M.S., Verschoor, J.A., 2008. Cholesteroid nature of free mycolic acids from $M$. tuberculosis. Chem.Phys.Lipids, 152, 95-103.

Beukes, M., Lemmer, Y., Deysel, M., Al Dulayymi, J.R., Baird, M.S., Koza, G., Iglesias, M.M., Rowles, R.R., Theunissen, C., Grooten, J., Toschi, G., Roberts, V.V., Pilcher, L., Van Wyngaardt, S., Mathebula, N., Balogun, M., Stoltz, A.C., Verschoor, J.A., 2011. Structurefunction relationships of the antigenicity of mycolic acids in tuberculosis patients (vol 163, pg 800, 2010) . Chem.Phys.Lipids., 164 175-175.

Fujiwara, N., Pan, J.W., Enomoto, K., Terano, Y., Honda, T., Yano, I., 1999. Production and partial characterization of anti-cord factor (trehalose-6,6 '-dimycolate) IgG antibody in rabbits recognizing mycolic acid subclasses of Mycobacterium tuberculosis or Mycobacterium avium FEMS Immunol. Med.Microbiol. 24, 141-149.

Furber, M., Taylor, R.J.K., Burford, S.C., 1986. Stereospecific diene synthesis using acetylene carbocupration; preparation of navel orangeworm pheromone and leukotriene analogues. J. Chem. Soc., Perkin Trans. 1, $1809-1816$. 
Hong, S., Cheng, T.Y., Layre, E., Sweet, L., Young, D.C., Posey, J.E., Butler, W.R., Moody, D.B., 2012. Ultralong C100 Mycolic Acids Support the Assignment of Segniliparus as a New Bacterial Genus. Plos One 7, e39017.

Hsu, F.-F., Soehl, K., Haas, A., 2011. Characterisation of mycolic acids from the pathogen Rhodococcus equi by tandem mass spectrometry with electrospray ionisation. Anal.Biochem., 409, 112-122.

Ireton, G.C., Greenwald, R., Liang, H., Esfandiari, J., Lyaschenko, K.P., Reed. S.G., 2010. Identification of Mycobacterium tuberculosis antigens of high serodiagnostic value. Clin.Vacc.Immunol., 17, 1539-1547.

Kowalski, K., Szewczyk, R., Druszczynska, M., 2012. Mycolic acids - potential biomarkers of opportunistic infections caused by bacteria of the suborder Corynebacterineae POSTEPY HIGIENY I MEDYCYNY DOSWIADCZALNEJ, 66, 461-468.

Koza, G., Theunissen, C., Al Dulayymi, J.R., Baird, M.S., 2009. The synthesis of single enantiomers of mycobacterial ketomycolic acids containing cis-cyclopropanes. Tetrahedron, 65, 10214-10229.

Laval, F., Lanéelle, M.A., Déon, C., Monsarrat, B., Daffé, M., 2001. Accurate Molecular Mass Determination of Mycolic Acids by MALDI-TOF Mass Spectrometry Anal. Chem., 73, 4537-4544.

Lemmer, Y., Thanyani, S. T., Vrey, P. J., Driver, C.H.S., Venter, L., van Wyngaardt, S., ten Bokum, A.M.C., Ozoemena, K.I., Pilcher, L.A., Fernig, D.G., Stoltz, A.C., Swai, H.S., Verschoor, J.A., 2009. DETECTION OF ANTIMYCOLIC ACID ANTIBODIES BY LIPOSOMAL BIOSENSORS. Editor: Duzgunes, N., LIPOSOMES, PT F Series: Methods in Enzymology : 464, 79-104.

Maekura, R., Nakagawa, M., Nakamura, Y., Hiraga, T., Yamamura, Y., Ito, M., Ueda, E., Yano, S., He, H., Oka, S., Kashima, K., Yano, I., 1993. Clinical evaluation of rapid serodiagnosis of pulmonary tuberculosis by ELISA with cord factor (Trehalose-6,6'dimycolate) as antigen purified from Mycobacterium tuberculosis. Am. Rev. Respir. Dis., $148,997-1001$

Mathebula, N.S.; Pillay, J.; Toschi, G.; Verschoor, J.V., Ozoemena, K.I., 2009. Recognition of anti-mycolic acid antibody at self-assembled mycolic acid antigens on a gold electrode: a potential impedimetric immunosensing platform for active tuberculosis Chem.Commun. 3345-3347.

Minnikin, D.E., 1982. Lipids: complex lipids, their chemistry, biosynthesis and roles. In 'The biology of the mycobacteria', Standford, J., Ed.; Academic Press, San Diego, , 95-184.

Morris, K., 2011. WHO recommends against innacurate tuberculosis tests. www. thelancet.com, 377. 
Ozoemena, K.I., Mathebula, N.S., Pillay, J., Toschi, G., Verschoor, J.A., 2010. Electron transfer dynamics across self-assembled N-(2-mercaptoethyl) octadecanamide/mycolic acid layers: impedimetric insights into the structural integrity and interaction with anti-mycolic acid antibodies Phys.Chem. Chem.Phys., 12, 345-357.

Pan, J.W., Fujiwara, N., Oka, S., Maekura, R., Ogura, T., Yano, I., 1999. Anti-cord factor (trehalose 6,6 '-dimycolate) IgG antibody in tuberculosis patients recognizes mycolic acid subclasses Microbiol.Immunol., 43, 863-869.

Sakai, J., Matsuzawa, S., Usui, M., Yano, I., 2001. New diagnostic approach for ocular tuberculosis by ELISA using the cord factor as antigen. Brit.J.Opthalmology 85, 130-133.

Salvado-Viader, J.M., Molina-Torres, C.A., Guerrero-Olazaran, M., 2007. Detection and identification of mycobacteria by mycolic acid analysis of sputum specimens and young cultures. J.Microbiol.Methods, 70, 479-483.

Schleicher, G.K., Feldman, C., Vermaak, Y., Verschoor, J.A., 2002. Prevalence of antimycolic acid antibodies in patients with pulmonary tuberculosis co-infected with HIV Clin.Chem.Lab.Medicine 40, 882-887.

Shui, G., Bendt, A.K., Jappar, I.A., Lim, H.M., Laneelle, M., Herve, M., Via, L.E., Chua, G.H., Bratschi, M.W., Rahim, S.Z.Z., Michelle, A.L.T., Hwang, S.H., Lee, J.S., Eum, S.Y., Kwak, H.K., Daffe, M., Dartois, V., Michel, G., Barry, C.E., Wenk, M.R., 2012. Mycolic acids as diagnostic markers for tuberculosis case detection in humans and drug efficacy in mice. EMBO Mol.Medicine 4, 27-37.

Song, S.H.; Park, K.U.; Lee, Jae Ho; et al., 2009. Electrospray ionization-tandem mass spectrometry analysis of the mycolic acid profiles for the identification of common clinical isolates of mycobacterial species J.Microbiol.Methods, 77, 165-177.

Steingart, K.R., Henry, M., Laal, S., Hopewell, P.C., Ramsay, A., Menzies, D., Cunningham, J., Weldingh, K., Pai, M., 2007. Commercial serological antibody detection tests for the diagnosis of pulmonary tuberculosis: a systematic review. PLoS Medicine, 4, 1041-1056.

Steingart, K.R., Dendukuri, N., Henry, M., Schiller, I., Nahid, P. Hopewell, P.C., Ramsay, A., Pai, M., Laal, S., 2009. Performance of purified antigens for diagnosis of pulmonary tuberculosis: a meta-analysis. Clin.Vacc.Immunol., 16, 260-276.

Thanyani, S.T., Roberts, V., Siko, D.G.R., Vrey, P., Verschoor, J.A., 2008. A novel application of affinity biosensor technology to detect antibodies to mycolic acid in tuberculosis patients J.Immunol.Methods, 332, 61-72.

Thuo, M.M., Reus, W.F., Nijhuis, C.A., Barber, J.R.,, Kim, C., Schulz, M.D., Whitesides, 2011. Odd-Even Effects in Charge Transport across Self-Assembled Monolayers. J.Am.Chem.Soc., 133, 2962-2975.

Uenishi, Y., Fujita, Y., Kusunose, N., Yano, I., Sunagawa, M., 2008. Comprehensive analysis of mycolic acid subclass and molecular species composition of Mycobacterium bovis BCG Tokyo 172 cell wall skeleton (SMP-105) J.Microbiol.Methods 72, 149-156. 
Verschoor, J., 2010. Mycolic acid antibody real-time inhibition test demonstrates that antimycolic acid antibodies are surrogate markers for active tuberculosis Trop.Med.Int.Health, $15, \mathrm{~S} 26-\mathrm{S} 26$.

Verschoor, J.A., Baird, M.S., Grooten, J.. 2012. Towards understanding the functional diversity of cell wall mycolic acids of Mycobacterium tuberculosis. Prog Lipid Res., 51,325339.

Watanabe, M; Aoyagi, Y; Ridell, M; Minnikin, D.E., 2001. Separation and characterization of individual mycolic acids in representative mycobacteria Microbiology 147, 1825-1837.

Watanabe, M; Aoyagi, Y; Mitome, H; Fujita, T.; Naoki, H.; Ridell, M.; Minnikin, D.E. , 2002. Location of functional groups in mycobacterial meromycolate chains; the recognition of new structural principles in mycolic acids Microbiology 148, 1881-1902.

Yassin A.-A. F., 2011. Detection and Characterization of Mycolic Acids and Their Use in Taxonomy and Classification ; Editors: Rainey, F.; Oren, A. Taxonomy of Prokaryotes Book Series: Methods in Microbiology, 38, 207-237. 